\title{
4. Objektivistische Theorien der Farben
}

Die Differenz zwischen einer objektivistischen und einer subjektivistischen Theorie der Farben ist an dem Kriterium der Geistabhängigkeit ausgemacht worden: für den Objektivismus existieren Farbtatsachen unabhängig von unseren Farbwahrnehmungen, während sie für den Subjektivismus ontologisch von diesen abhängen. Dieser Unterschied läßt sich nun auf die Modalität des Bikonditionals (BK) übertragen: nach objektivistischer Lesart ist diese These nicht epistemisch notwendig. Denn das heißt nichts anderes, als daß Farbtatsachen nicht logisch-begrifflich mit Farbwahrnehmungen von Subjekten verbunden sind: sie genießen Geistunabhängigkeit. Da laut der Individuationsthese (IND) trotzdem eine bestimmte Beziehung zu unseren Sinneserfahrungen bestehen soll, werden Farben als repräsentierte Eigenschaften angesehen, so daß die These ( $\left.\mathrm{IND}_{\mathrm{R}}\right)$ als Ausgangspunkt dient: Farben sind die von Farbwahrnehmungen repräsentierten Eigenschaften. Aus den bisherigen Überlegungen ergibt sich damit nun die allgemeine Bestimmung, daß objektivistische Positionen sich dadurch auszeichen, daß sie die These (REP) als Grundlage für ihre Theorie über Farben nehmen. Sie gehen also von der systematischen Veridizität unserer Farbwahrnehmungen aus. Farben als repräsentierte Eigenschaften zeigen wirklich diejenigen Merkmale, die den repräsentationalen Aspekt der Intuitiven Farbkonzeption ausmachen. Vor diesem Hintergrund lassen sich nun Farbwahrnehmungen ohne Gefahr eines Zirkels über ihren Gehalt typenindividuieren, da die Farbeigenschaften objektiv charakterisiert werden können.

Zusätzlich wird der Farbobjektivismus - als eine Subtheorie des ontologischen Realismus - damit die Annahme verbinden, daß Farbwahrnehmungen auch repräsentationale Veridizität zeigen können: entweder dann, wenn sie unter Normalbedingungen gemacht werden - oder sogar immer, wenn es sich gar nicht um repräsentationale, sondern vielmehr um informationale Zustände handelt (wobei es in diesem Fall vielleicht besser ist, nicht von einer ,repräsentationalen Veridizität“ zu sprechen). Wird die letztere, stärkere These vertreten, handelt es sich um einen Informationalismus, der jedoch erst weiter unten thematisiert werden soll. Für den Moment genügt es, sich auf den Farbobjektivismus zu konzentrieren, der Repräsentationa- 
lität der Farbwahrnehmungen annimmt. Diese können nun nach dem eben Gesagten in allen ihren Aspekten ihres repräsentationalen Gehaltes veridisch sein. Motiviert ist eine solche Position natürlich durch den Umstand, daß sie eine ganz einfache Erklärung für unsere gewöhnliche Ansicht über Farben liefern kann, sofern diese die Seite der Farbrepräsentation betrifft: unsere naiven Auffassungen über Farben als repräsentierte Eigenschaften entsprechen eben den Tatsachen. Zusätzlich folgt sie auch der Objektivitätsintuition des Direkten Realismus und verbindet diese auf eine unkomplizierte Weise mit der von uns mittels Introspektion gewonnenen Einsicht, daß Farben die von Farbwahrnehmungen repräsentierten Eigenschaften sind.

Damit fügt sich die objektivistische Konzeption der Farben leicht in ein realistisch physikalistisches Weltbild ein, da sie Farben nicht anders als Formen - das heißt also: die sekundären nicht anders als die primären Qualitäten - behandelt. Da die Naturalisierung letzterer keine ernsthaften Probleme aufzuwerfen scheint, kann dies auch für erstere erhofft werden - natürlich nur unter der Voraussetzung, daß es einer Theorie der Farben tatsächlich gelingen kann, der Intuitiven Farbkonzeption auf diese Weise gerecht zu werden. Im folgenden sollen die einzelnen Varianten des Objektivismus daraufhin überprüft werden. Zuerst sollen die beiden Alternativen des Farbphysikalismus - der Schwache und der Starke Physikalismus - vorgestellt und disktutiert werden. Die Einbeziehung der Phänomenalität von Farben führt dann zu der dazu orthogonalen Unterscheidung des Aktualitäts- und des Notwendigkeitsobjektivismus, so daß insgesamt vier verschiedene, physikalistische Positionen differenziert werden können. Zudem soll schließlich auf zwei Abweichungen vom üblichen Farbphysikalismus eingegangen werden: auf den Informationalismus und auf den Primitivismus.

\subsection{Der Farbphysikalismus im allgemeinen}

\subsubsection{Die Definition des Farbphysikalismus}

Der repräsentationale Aspekt der Intuitiven Farbkonzeption beinhaltet un- 
ter anderem das Merkmal der Objektivität. Folgt man nun dem Weltbild eines globalen Realismus, gepaart mit einem Physikalismus, dann ist es unvermeidlich, objektive Eigenschaften nicht auch als physikalische anzusehen. Selbst der Primitivismus wird sich dieser Einsicht beugen müssen, will er nicht in einen mysteriösen Eigenschaftsdualismus verfallen. Doch auch die anderen Merkmale des repräsentationalen Aspektes müssen auf die Farben zutreffen, damit die systematische Veridizität gewährleistet ist. Somit sind die von uns repräsentierten Farben nichts anderes als objektive, physikalisch beschreibbare Eigenschaften von Gegenständen in der Außenwelt, die das Auftreten unserer Farbwahrnehmungen erklären können und zudem einen intrinsischen Charakter aufweisen. Dieser These entsprechen auch die üblicherweise gegebenen (wenn auch weniger ausführlichen) Definitionen des Farbphysikalismus: ${ }^{1}$

„Physicalists claim that colors are physical properties (for instance, that green is a certain property of selectively reflecting incident light).“ (Einleitung zu Byrne \& Hilbert (1997a): xii)

„By realism about colour I mean the view that colours are properties of the natural world of space, time, matter and energy. More than that: Realism is the thesis that colours are, in some substantial sense which is easy enough to grasp but rather tricky to spell out, physical properties - that is, properties belonging to the same family as shape, temparature and texture characterizing the public, inanimate world around us." (K. Campbell (1993): 250)

Die drei Grundpfeiler des Farbphysikalismus sind damit die Repräsentierbarkeit, die Externalität und die Objektivität (in Form der Physikalität) der Farbeigenschaften. Hinzu tritt die durch die allgemeine Akzeptanz des ontologischen Realismus sehr plausibel gemachte Annahme, daß unsere Farb wahrnehmungen im Regelfall auch repräsentationale Veridizität zeigen:

1 Der Farbphysikalismus sollte nicht mit der generellen ontologischen These des globaben Physikalismus verwechselt werden. Denn man kann, global betrachtet, Physikalist sein, ohne einen Farbphysikalismus zu vertreten: insbesondere, wenn man Farben nicht als externe Eigenschaften ansieht. Und auch umgekehrt muß das Implikationsverhältnis nicht unbedingt bestehen: ein Farbphysikalist kann ohne weiteres zulassen, daß auch nicht-physikalische, naturalistische Eigenschaften (wie beispielsweise biologische) einen wesentlichen Platz in der Beschreibung der Welt einnehmen. 
und zwar genau dann, wenn Normalbedingungen vorliegen. Es ist zwar immer noch denkbar, daß letzteres nie der Fall sein könnte, aber dies wäre - auch vor dem Hintergrund der allgemeinen Unbestimmtheit der Wahrnehmung - doch sehr unwahrscheinlich. Die Physikalität der Farben als objektive Eigenschaften muß nun jedoch nicht unbedingt bedeuten, daß sie Teil der von der Physik behandelten Eigenschaften sind. Vielmehr genügt es, wenn sie allein auf solchen supervenieren und somit physikalisch (oder im weiteren Sinne: naturwissenschaftlich) beschreibbar sind. Hierin spiegelt sich der Umfangsunterschied der Begriffe der Physikalität - oder physikalischen Beschreibbarkeit - und der Physik-Immanenz wieder.

Physik-immanente Eigenschaften sind immer (komplexe) physikalische Größen oder Arten (physical kinds), die eine ausgezeichnete Rolle innerhalb der Physik einnehmen. Sie lassen sich mithilfe physikalischer Gesetze auf fundamentale, physikalische Größen reduzieren. Physikalität beinhaltet nicht unbedingt diese Reduzibilität. Physikalische Eigenschaften müssen zwar immer auf physik-immanenten supervenieren, aber ihre Individuation kann auch mithilfe von Faktoren oder Gemeinsamkeiten passieren, die der Physik außenvorliegen. Bei diesen Individuationsmerkmalen kann es sich zum Beispiel um funktionale Beschreibungen handeln, aber auch um subjektrelative (wobei Subjektrelativität nichts mit Geistabhängigkeit zu tun hat). Herzen können zwar physikalisch vollständig beschrieben werden, aber das ihnen allen gemeinsame und wesentlich zukommende Merkmal kann erst über die Funktion, Blut zu pumpen, angegeben werden; und die Klasse der mir gehörenden Dinge ist subjektrelativ spezifiziert, obwohl es sich wiederum um physikalisch beschreibbare Gegenstände handeln wird. Es genügt nun, wenn Farben physikalisch sind; sie müssen nicht noch zusätzlich physik-immanente Eigenschaften sein. Dies zeigt sich ebenfalls in dem Gedanken, daß Intrinsität auch die Supervenienz auf intrinsische Eigenschaften von externen Gegenständen erlaubt (die fundamental realisierenden, intrinsischen Eigenschaften sind nach dem Physikalismus immer physik-immanent). Mit dieser Einsicht verändert sich die Bestimmung der physikalistischen Position ein wenig.

„Physicalism about color is, to a first approximation, the view that colors are physical properties that we sometimes veridically perceive objects to possess. The 'physical' is a notoriously slippery notion, but fortunately those problems are 
not relevant here. The leading idea behind physicalism is not so much that colors are physical properties, but rather that colors are to be identified with properties whose natures (a) are specifiable in ways that do not employ our color concepts, and (b) are not constituted by relations to the psychological states of perceivers." (Einleitung zu Byrne \& Hilbert (1997a): xxii)

Interessanterweise ist dabei - vor dem Hintergrund der naturalistischen Doktrin - die Physikalität mit Bezug auf die alternativen Theorien der Farben eigentlich der am wenigsten charakteristischste Aspekt des Farbphysikalismus. Denn es ist unklar, ob es letztlich überhaupt Eigenschaften in der Welt gibt, die sich einer naturalistischen Erklärung entziehen werden. Die einzigen Farbtheorien, die vielleicht die Physikalität der von ihnen beschriebenen Farben leugnen können, sind solche, die diese in einen engen, ontologischen Zusammenhang mit dem phänomenalen Charakter von Farbwahrnehmungen bringen und gleichzeitig dessen Naturalisierbarkeit verneinen. Doch selbst Qualia finden heutzutage wieder verstärkt ihren Platz innerhalb funktionalistischer oder repräsentationalitischer Positionen, so daß vielleicht bald, wenn doch von keiner Physikalisierung, so doch wenigstens von einer Naturalisierung derselben gesprochen werden könnte. Jedenfalls weisen die Debatten darauf hin, daß die Physikalität der Farben nicht (mehr) der herauszuhebende Ausgangspunkt der Kontroverse zwischen Objektivismus und Subjektivismus ist. Vielmehr stehen die drei anderen, eben benannten Aspekte im Vordergrund, und dabei insbesondere die Frage nach der externen Lokalisierung von Farbinstanzen. Neben der Repräsentierbarkeit, der Objektivität und der Möglichkeit repräsentationaler Veridizität ist also vor allem die Externalität ein Kennzeichen des Farb physikalismus.

Dies zeigt sich auch darin, daß nach der Akzeptanz dieser Merkmale eine Physikalität der Farben nicht mehr vermieden werden kann. Nimmt man den Naturalismus ernst, dann ist die Beschaffenheit von Gegenständen in der äußeren Welt angemessen und vollständig durch die Physik beschreibbar. Nur relationale oder dispositionale Eigenschaften hinsichtlich mentaler Zustände können vielleicht eine Ausnahme darstellen. Die physikalistische Identifikation von Farben mit einer dieser beiden Eigenschaftsarten ist für den Objektivismus jedoch unmöglich, da er ontologische Geistunabhängigkeit fordert. Somit ist die physikalische Beschreibbarkeit der 
Farben - vor dem Hintergrund des globalen Physikalismus hinsichtlich der Außenwelt - eher eine Konsequenz, denn ein bestimmender Aspekt, des Farbphysikalismus. Dennoch ist die Klasse $K_{\mathrm{F}}$ der Farben natürlich - laut der Analysethese (ANA) - mit einer wie auch immer zu bestimmenden Klasse $K_{\mathrm{P}}$ physikalischer Eigenschaften gleichzusetzen, wobei die einzelnen Farbeigenschaften $F_{\mathrm{i}}$ aus $K_{\mathrm{F}}$ jeweils ganz bestimmten (möglicherweise komplexen) physikalischen Eigenschaften $P_{\mathrm{i}}$ aus $K_{\mathrm{P}}$ entsprechen, und es sich bei den $P_{\mathrm{i}}$ zudem um extern instantiierbare Eigenschaften handeln muß. Die Farbe Rot ist so beispielsweise mit der physikalischen Eigenschaft $P_{\text {ROT }}$ identisch; und vergleichbares gilt für alle anderen Farbeigenschaften.

Für den Physikalisten - wie für jeden Farbtheoretiker - stellen sich nun zwei Aufgaben. Einerseits sollte er das den Umfang der Klasse $K_{\mathrm{P}}$ definierende Merkmal $P$ spezifizieren: also eine intensionale oder extensionale Definition der Klasse der Farbeigenschaften liefern. Andererseits fordert man von ihm, die Natur der einzelnen Eigenschaften $P_{\mathrm{i}}$ exakt bestimmen, deren konkrete Zuordnung zu den Farbeigenschaften angeben und, wenn möglich, auch erklären zu können. ${ }^{2}$ Die Eigenschaften $\mathrm{P}_{\mathrm{i}}$ müssen dabei, ebenfalls wie die Farben, eine Determinablen-Determinanten-Struktur bilden. Die üblicherweise verwendete Strategie ist es, zuerst die zweite Fragestellung zu erörtern, um mit den gewonnenen Erkenntnissen dann die erste angehen zu können. Hat man nämlich in physikalischen Begriffen bestimmen können, was es heißt, daß ein Gegenstand beispielsweise die Farbe Rot oder die Farbe Gelb instantiiert, dann wird sich möglicherweise ein gemeinsames Merkmal der betreffenden Farbeigenschaften finden lassen, welches das allgemeinere Charakteristikum, eine Farbe zu sein, ausmacht.

2 Letztlich wird die Angabe der einzelnen Eigenschaften $P_{\mathrm{i}}$ eine Aufgabe der Colorimetrie, und nicht der Philosophie, sein. Für die philosophische Diskussion des Farbphysikalismus reicht es aus, die Durchführbarkeit dieser Aufgabe zu bewerten. Der Physikalist muß nicht in der Lage sein, jeder Farbe konkret eine bestimmte physikalische Eigenschaft zuzuordnen (allein schon deswegen, da es eine Unmenge von Farbeigenschaften gibt); er sollte aber auf die Möglichkeit verweisen können, daß zu jeder beliebigen Farbe durch empirische Nachforschungen eine physikalische Eigenschaft sich finden und angeben läßt. Anders ist es mit der Klasse $K_{\mathrm{P}}$ an sich: hier sollte der Physikalist in der Lage sein, ein angemessen genaues Individuationskriterium $P$ zu formulieren, welches darüber entscheidet, ob eine gegebene Eigenschaft Mitglied der Klasse ist oder nicht. 
Dieser Gedankengang mündet damit in der folgenden, an (ANA) orientierten und für die weitere Diskussion anwendbaren Definition des Farbphysikalismus (erst einmal ganz allgemein auf alle aktual existierenden, farbwahrnehmende Subjekte bezogen):

(PHY) (i) Die Thesen $\left(\mathrm{IND}_{\mathrm{R}}\right)$ gilt: alle unsere aktual möglichen Farb wahrnehmungen repräsentieren Eigenschaften $F_{\text {i }}$, die der Klasse der Farbeigenschaften $K_{\mathrm{F}}$ zugehören.

(ii) Die These (REP) gilt: unsere Farbwahrnehmungen sind systematisch veridisch.

(iii) Unter Normalbedingungen liegt auch repräsentationale Veridizität vor.

(iv) Die These (ANA) gilt für physikalische Eigenschaften $P_{\mathrm{i}}$ mit dem (im weiten Sinn) physikalisch beschreibbaren Merkmal $P$.

Die Konkretisierung der physikalistischen Position erfordert nun zumindest die Spezifierung des Individuationsmerkmales $P$ der Klasse der Farbeigenschaften: also dessen, was allen Farben als gemeinsames Charakteristikum zukommt. Darüberhinaus sollte auch prinzipiell aufgezeigt werden, wie es unter der Zuhilfenahme empirischer Untersuchungen möglich ist, zu jeder Farbe $F_{\mathrm{i}}$ die passende Eigenschaft $P_{\mathrm{i}}$ anzugeben. Und außerdem fehlt noch eine Einbettung des Merkmales der Erklärungskraft, wobei dies aufgrund der Repräsentiertheit kein allzu großes Problem darstellen sollte. Bevor jedoch die einzelnen Varianten des Farbphysikalismus diskutiert werden können, ist es sinnvoll, sich einer Minimalanforderung an jede Bestimmung der Farbeigenschaften $P_{\mathrm{i}}$ klar zu werden.

\subsubsection{Die Individuation der P-Eigenschaften}

Eine physikalistische Theorie muß in der Lage sein, die These (PHY) noch mit weiterem Inhalt auszufüllen: indem beispielsweise die einzelnen Eigenschaften $P_{\mathrm{i}}$ und das ihnen gemeinsame Merkmal $P$ benannt werden. Nun ist die These $\left(\operatorname{IND}_{\mathrm{R}}\right)$ als ein erstes Individuationskriterium für die Farben eingeführt worden. Deswegen bietet es sich an, zur Identifizierung der 
$P_{\mathrm{i}}$ sich der aktualen Repräsentierbarkeit von Farben $\mathrm{zu}$ bedienen. Welche Eigenschaften ein mentaler Zustand repräsentiert, kann wiederum auf zwei Wegen festgestellt werden: entweder durch Introspektion auf den betreffenden Zustand, oder durch die Anwendung einer Theorie der Repräsentation auf denselben. Dabei kann eine Art von Arbeitsteilung festgestellt werden. Während die Introspektion für uns zwar als letztlich entscheidende Instanz dafür gelten sollte, wann Gehaltsunterschiede vorliegen, gewährleistet nur eine empirisch unterstützte Theorie auch die Verbindung $\mathrm{zu}$ den jeweils wahrgenommenen, physikalischen Farbeigenschaften in der Welt. Denn im introspektiven Charakter unserer Wahrnehmungen ist uns die physikalische Konstitution von Farben selbst nicht gegeben. Dagegen kann eine objektive Theorie der Repräsentationalität von Wahrnehmungen dabei helfen, den repräsentierenden Zustand mit in der Außenwelt instantiierten Eigenschaften in Beziehung $\mathrm{zu}$ setzen, so daß deren empirische Erforschung möglich wird. Bei dieser Relation handelt es sich, wie bereits erwähnt, um eine nomologische Korrelation unter Normalbedingungen: erst wenn diese zwischen einem Eigenschafts- und einem Zustandstypes vorliegt, handelt es sich um eine Repräsentationsbeziehung.

Betrachtet man nun das laut Farbphysikalismus mögliche Auftreten von unter normalen Umständen gemachten Farbwahrnehmungen eines ganz bestimmten Types, so kann man die wahrgenommenen Gegenstände auf eine gemeinsame, physikalische Eigenschaft untersuchen, die mit der fraglichen Sinneserfahrung kontrafaktisch verbunden sind; mit dieser Eigenschaft hat man dann den Kandidaten gefunden, der mit der betreffenden, wahrgenommenen Farbe gleichzusetzen ist. Es bleibt jedoch immer noch das Problem bestehen, wie man die einzelnen Farbwahrnehmungen als einem bestimmten Typus zugehörig individuieren kann. Auch hier kann erneut die These $\left(\mathrm{IND}_{\mathrm{R}}\right)$ weiterhelfen: genau die Wahrnehmungen, die ein- und dieselbe Eigenschaft repräsentieren, bilden einen eigenen Typus. Doch darf diesmal die Individuierung nicht über eine Theorie der Repräsentation erfolgen, da dann die folgende Zirkularität aufträte: um mithilfe der Farbwahrnehmungen die korrekt repräsentierten Eigenschaften $P_{\mathrm{i}}$ in der Welt zu identifizieren, können erstere natürlich nicht wiederum mithilfe letzterer bestimmt werden (ein ähnliches Problem ergab sich bei der ohne Rückgriff auf Farben durchzuführenden Bestimmung von Farbwahrnehmungen). Aber es 
gibt ja noch die sich der Introspektion bedienenden Alternative zu der empirisch-theoretischen Individuation von Wahrnehmungen als repräsentationale Zustände. Welche Eigenschaft eine Sinneserfahrung repräsentiert (oder zumindest, wann Gehaltsdifferenzen vorliegen), kann nämlich ebenso introspektiv erkannt werden: und zwar mittels des phänomenalen Aspektes des introspektiven Charakters.

Genau dieser Umstand wird durch die Aktualitätsthese unter Normalbedingungen ausgedrückt, welche der Farbphysikalismus als eine Position, die Farben als repräsentierte Eigenschaft ansieht, üblicherweise akzeptieren wird. Der These $\left(\mathrm{ACT}_{\mathrm{NB}}\right)$ zufolge gilt nun, daß ein Gegenstand genau dann die Farbe $F_{\mathrm{i}}$ instantiiert, wenn er unter Normalbedingungen in einem Betrachter faktisch immer $F_{\mathrm{i}}$-phänomenale Farbwahrnehmungen hervorruft. $^{3}$ Dem Farbphysikalisten ist es jetzt möglich, an die Stelle der Farbeigenschaft $F_{\mathrm{i}}$ die gesuchte, physikalische Eigenschaft $P_{\mathrm{i}}$ zu setzen. Mithilfe des phänomenalen Aspektes des introspektiven Charakters lassen sich dann die farbigen Gegenstände unter günstigen Bedingungen in Gruppen teilen, die Gemeinsamkeiten oder Verschiedenheiten in ihrer Farbigkeit widerspiegeln. Kennt man nun die physikalische Beschaffenheit von diesen Gegenständen, so sollte sich für jede Farbe ein gemeinsames physikalisches Merkmal finden lassen (falls es dies überhaupt gibt). Dabei wird es sich dann um das die Klasse $K_{\mathrm{P}}$ der Farbeigenschaften definierende Merkmal $P$ handeln. Es ist dementsprechend auch nicht überraschend, daß innerhalb der naturwissenschaftlichen Erforschung der Farbigkeit von Gegenständen durch die Colorimetrie (das heißt: der Wissenschaft, die sich mit der Identifizierung der für Farbwahrnehmungen kausal oder explanatorisch verantworlichen Eigenschaften beschäftigt) genau die eben skizzierte und letztlich in der These $\left(\mathrm{ACT}_{\mathrm{NB}}\right)$ gipfelnde Methode für die Individuierung von Farbeigenschaften angewandt wird (und zwar mithilfe der sogenannten Metamerik oder Isomerik - vgl. Hardin (1988): 28).

Die These $\left(\mathrm{ACT}_{\mathrm{NB}}\right)$ kann jedoch nur als ein vorläufiges Individuationskriterium fungieren, da sie Farben über mentale Zustände klassifiziert, der

3 Die These $\left(\mathrm{ACT}_{\mathrm{NB}}\right)$ gilt natürlich nur für die von $\left(\mathrm{IND}_{\mathrm{R}}\right)$ erfaßten, das heißt, die tatsächlich repräsentierbaren Farbeigenschaften. Sollte es zudem nicht-wahrnehmbare Farben geben (für die unser Auge zum Beispiel nicht empfindlich genug ist), dann können wir von diesen natürlich auch nicht - oder nur ungenau, unbestimmt - Kenntnis erlangen. 
Farbphysikalismus jedoch als Objektivismus jede ontologische Geistabhängigkeit vermeiden muß. Die Aktualitätsthese wird zwar aller Voraussicht nach faktisch ihre Gültigkeit behalten, indem sie eine kontingente Verbindung zwischen Farbtatsachen in der Welt und Farbwahrnehmungen in Betrachtern behauptet, sie darf aber nicht einen wesentlichen Aspekt der Natur der Farben bestimmen. Man könnte meinen, daß dies für einen Objektivismus ${ }_{\mathrm{NEC}}$ anders aussieht, da dieser zudem die Notwendigkeitsthese unter Normalbedingungen annimmt, so daß Gehalt und Quale niemals auseinandergehen können. Doch auch dann liegt keineswegs eine Subjektabhängigkeit vor. Diese ist erst bei einer epistemischen Notwendigkeitsbeziehung gegeben, welche der Objektivist jedoch ablehnt. Denn für den Farbphysikalisten verläuft die Begründungsrichtung des Bikonditionals (BK) offensichtlich immer von links nach rechts: ein Gegenstand erscheint uns normalerweise als rötlich, weil er rot ist, das heißt, die entsprechende Eigenschaft $P_{\text {ROT }}$ instantiiert - und nicht etwa umgekehrt. In jedem Fall kann die Aktualitätsthese $\left(\mathrm{ACT}_{\mathrm{NB}}\right)$ in der aktualen Welt dabei helfen, den Farbwahrnehmungstypen jeweils konkret eine repräsentierte Eigenschaft $P_{\mathrm{i}}$ zuzuordnen, weil Farben faktisch genau die Eigenschaften sind, die unter Normalbedinungen in einem Betrachter eine Sinneserfahrung mit einem farbspezifischen Quale hervorrufen.

\subsubsection{Formen des Farbphysikalismus}

Zusammenfassend läßt sich nun sagen, daß die Physikalität der einzelnen Farbeigenschaft - das heißt: ihre vollständige Beschreibbarkeit in der durch die Physik vorgebenen Terminologie - nicht das entscheidende oder kontrovers disktutierte Merkmal des Farbphysikalismus ist. Dieser zeichnet sich vielmehr durch die Repräsentierbarkeit, Objektivität und die Externalität von Farben sowie die grundsätzliche Annahme der repräsentationalen Veridizität aus. Die Physikalität ist dagegen eigentlich eher eine Folge daraus, daß Farbeigenschaften als Bestandteil der raum-zeitlichen Realität angesehen werden. Trotzdem steht natürlich für einen Farbphysikalisten wie auch für die meisten anderen, philosophischen Positionen über Farben - die Frage im Vordergrund, welche konkrete, physikalische Natur Farben 
aufweisen. Hierbei geht es sowohl um die Identifizierung der einzelnen Farbeigenschaften als auch um die Bestimmung des ihnen gemeinsamen Merkmales, also des Farbeseins als solches. ${ }^{4}$ Das heißt, nicht nur die Extension der mit der Klasse der Farben identischen Klasse physikalischer Eigenschaften steht zur Debatte, sondern auch die Suche nach einem alle Farben - und nur diese - auszeichnenden Charakteristikum. Die detaillierte Bestimmung ersterer ist weniger die Angelegenheit der Philosophie, sondern vielmehr der empirischen Forschung. Doch die Philosophie muß auf der Basis naturwissenschaftlicher Erkenntnisse - hierfür erst die Grundlage schaffen, indem sie das die Menge der Farben definierende Merkmal $P$ zu spezifizieren versucht. Dabei kann es $\mathrm{zu}$ erheblichen Differenzen zwischen verschiedenen Formen des Farbphysikalismus kommen.

Die Analyse der allgemeinen physikalischen Beschaffenheit der Farben ist nun eine Sache; die Frage, warum die ausgewählten Eigenschaften, deren physikalische Konstitution bestimmt werden soll, überhaupt als Farben anzusehen sind, eine ganz andere. Es gibt dabei die zwei Möglichkeiten, daß entweder bestimmte Aspekte der physikalischen Beschaffenheit wesentlich zu ihrer Natur dazugehören (oder diese sogar vollständig erschöpfen), oder aber daß die konkrete Physikalität der Farben diesen nur akzidentiell zukommt. Im letzteren Fall werden dann irgendwelche, durch die physikalische Konstitution realisierte Eigenschaften höherer Ordnung das Gemeinsame der Farben ausmachen (die offensichtlichsten Kandidaten sind Dispositionen und Disjunktionen). Die beiden Alternativen unterscheiden sich also darin, ob das allen Farbeigenschaften gemeinsam zukommende und physikalisch beschreibbare Merkmal von einer ausgezeichneten, besonderen Relevanz oder Bedeutung für die Physik als Naturwissenschaft ist, das heißt: ob Farben aufgrund ihrer physikalischen Beschaffenheit als physikalische Größe oder Art gelten, oder nicht. Eine physikalische Art (physical kind) ist dabei, ganz grob, ein solcher Eigenschaftstypus, der sich mithilfe von Gesetzen auf fundamentalerer physikalische Größen und Kon-

4 Die physikalische Beschreibung einer konkreten Farbe wird dabei sowohl qualitativ als auch quantitativ sein: denn sie wird daraus bestehen, bestimmten physikalischen Meßgrößen einen Wert zuzuordnen. Eine philosophische Betrachtung der Farben kann sich jedoch mit deren allgemeiner, qualitativer Bestimmung zufrieden geben. 
stanten reduzieren läßt (wie zum Beispiel Geschwindigkeit als zurückgelegte Strecke pro Zeiteinheit definiert werden kann).

Es geht also um die Frage, ob Farben bloß physikalisch beschreibbare oder zusätzlich auch physik-immanente Eigenschaften sind. Damit verbunden ist natürlich direkt die Fragestellung, ob Farben tatsächlich kausal wirksam sind oder nur eine Rolle im Erklärungsnetz der supervenierenden Eigenschaften spielen. Das beide Alternativen zu einer physikalistischen Theorie der Farben führen, ist bereits argumentiert worden. Hilbert beispielsweise, der Farben als Reflektanzdispositionen bestimmt, sieht zwar seine Position zu Recht als eine physikalistische an, auch wenn die betreffenden Farbeigenschaften keine Physik-Immanenz aufweisen:

\begin{abstract}
„Although I have been calling my account of what colors are a physicalist account, this may be slightly misleading. If to be a physicalist about colors implies believing that they can be reduced to interactions of the fundamental particles and laws of quantum mechanics, then my analysis of colors is not a physicalist analysis. I do not see how to identify reflectances with any of the fundamental enitites of quantum mechanics or any non-disjunctive collection of such entities. In any event, my main purpose is to show that colors are objective properties of external objects and establishing their identity with reflectances satisfies this object.“ (Hilbert (1987): 100)
\end{abstract}

Es kann gut der Fall sein, daß das allen Farben Gemeinsame nicht so einfach durch die Physik erfaßbar ist, weil es zum Beispiel als wesentlichen Zug einen gewissen Grad an Subjektrelativität (oder allein auf den Menschen bezogen: Anthropozentrik) beinhaltet. Und Subjekte als solche haben eindeutig keinen Platz innerhalb der Physik, so daß ein subjektbezogenes Charakteristikum der Farben nicht physik-immanent beschreibbar ist. Mithilfe der Physik-Immanenz und der Subjektrelativität lassen sich nun zwei Formen des Farbphysikalismus unterscheiden. Der Starke Physikalismus bestimmt die Klasse der Farbeigenschaften ohne jede Bezugnahme auf faktisch oder auch nur möglich existierende Subjekte; stattdessen wird dem allen Farben gemeinsamen Charakteristikum $P$ - als einer (komplexen) physikalischen Größe oder Art - ein ganz bestimmter Platz innerhalb der Physik zugewiesen. Farben sind hiernach wesentlich physik-immanente Eigenschaften ohne jede Subjektrelativität. Diese Option entspricht sicherlich am ehesten einer gewöhnlichen Vorstellung von einer physikalistischen 
Theorie der Farben.

Im Gegensatz dazu fordert der Schwache Physikalismus nur ein subjektrelatives, gemeinsames Merkmal $P$ für die Farben, welches zwar als solches keine ausgezeichnete Relevanz für die Physik hat, aber sich trotzdem physikalisch (oder naturwissenschaftlich) beschreiben läßt. Farben sind demnach subjektrelative Eigenschaften externer Gegenstände; ihre konkrete, physikalische Konstitution, die die Farbigkeit realisiert, kommt ihnen nur akzidentiell zu. Prominente Beispiele für diese Alternative stellen der objektivistische Dispositionalismus sowie der Disjunktivismus dar. Mit dem Starken und dem Schwachen Physikalismus sind jedoch noch nicht alle Möglichkeiten für einen Farbphysikalismus ausgeschöpft. Freilich schließen sich die beiden Kriterien der Physik-Immanenz und der Subjektrelativität aus den oben genannte Gründen aus.

Es ist aber vielleicht auch möglich, eine Position $\mathrm{zu}$ formulieren, die Farben zwar als externe, physikalisch realisierte Eigenschaften ansieht, aber ihnen keines der beiden Charakteristika zukommen läßt. So beläßt die nicht-dualistisch verstandene Varianate des Primitivismus den Farben ihre Physikalität, während er sie weder auf Kombinationen von physikalischen Größen oder Arten reduziert, noch eine Subjektrelativität als wesentlichen Bestandteil ihrer Natur betrachtet (der Primitivismus ähnelt damit vielleicht eher dem Starken als dem Schwachen Physikalismus). Der Primitivismus teilt dabei mit dem Schwachen Physikalismus die Nicht-Reduzierbarkeit der Farben auf für die Naturwissenschaft Physik bedeutsame Eigenschaften; die beiden Positionen differieren hingegen in dem Umstand eines bestehenden oder nicht-bestehenden, wesentlichen Subjektbezuges. Es ist zwar theoretisch möglich, daß es noch weitere Varianten eines Farb physikalismus geben kann, aber die in der Literatur vorgeschlagenen Alternativen sind hiermit erschöpft. ${ }^{5}$

5 Der Informationalismus - wenigstens der von Averill (1982) - kann als eine Variante des Starken Physikalismus gelten. Der Farbobjektivismus umfaßt zudem noch den dualistischen Primitivismus, der im Zusammenhang mit der nicht-dualistischen Alternative besprochen werden soll. 


\subsection{Der Starke Physikalismus}

Dem Starken Physikalismus zufolge sind Farben physik-immanente Eigenschaften. Das heißt, sie bilden selbst eine genuine physikalische Art, die sich auf bekannte Größen der Physik reduzieren läßt und deren Natur keinerlei Subjektrelativität zeigt. Die Farbigkeit (oder das Farbesein) $P$ ist somit eine physik-relevante Determinable, die einzelnen Farbeigenschaften $P_{\mathrm{i}}$ deren Determinanten: die konkrete physikalische Konstitution ist den Farben somit wesentlich. Aus dieser Bestimmung ergeben sich direkte Konsequenzen für die weiteren Merkmale des repräsentationalen Aspektes. Zuerst einmal werden Farben als intrinsische Eigenschaften der betreffenden Gegenstände verstanden, da Dispositionen oder Relationen zu Subjekten von vorneherein ausscheiden und Farben sicherlich auch nicht als externe Relationen zwischen Gegenständen anzusehen sind (interne Relationen sind vielleicht nicht ausgeschlossen). Daneben zeichnen sich alle physik-immanenten Eigenschaften dadurch aus, daß ihnen kausale Wirksamkeit zukommt, weil die Gesetze der Physik letzten Endes nichts anderes als Kausalgesetze sind, und die physikalisch beschreibbaren NaturgesetzmäBigkeiten somit kausaler Natur. Dies gilt nicht nur für die fundamentalen physikalischen Eigenschaften, sondern auch für die aus diesen zusammensetzbaren makroskopischen Größen. Die Wechselwirkungen zwischen Körpern mittlerer Größe und Lichtwellen lassen sich zwar durch die Wechselwirkungen zwischen den einzelnen Materie- und Lichtteilchen vollständig und angemessen erklären, doch diese Reduzierbarkeit beläßt der makroskopischen Erklärungsebene trotzdem ihre volle Gültigkeit und Kausalität. Und in vielen Fällen mag es sogar für die verfolgten Absichten günstiger zu sein, die makro-physikalische der mikro-physikalischen Beschreibung vorzuziehen: wie es insbesondere für die Farbproblematik anzunehmen ist, da sie sich vor allem mit mittelgroßen Gegenständen als Farbträgern beschäftigt.

Damit ergibt sich aber, daß der Starke Physikalismus, wie es scheint, allen fünf Merkmalen des repräsentationalen Aspektes des introspektiven Charakters von Farbwahrnehmungen Rechenschaft ablegen kann. Somit ist die systematische Veridizität von Farbwahrnehmungen gewährleistet. Nach diesen allgemeinen Ausführungen ist es an der Zeit, konkrete Ausformun- 
gen des Starken Physikalismus zu betrachten und zu diskutieren. Von besonderem, historischen Interesse ist dabei der Vorschlag, Farben entweder mit einzelnen, charakteristischen Wellenlängen oder aber mit bestimmten, komplexen Wellenlängenverteilungen von Licht gleichzusetzen. Doch die von Land durchgeführten, colorimetrischen Experimente haben solch einer Auffassung schnell ihre Grenzen gezeigt: es gibt keine noch so beschaffene, einfache Korrelation zwischen Farben (oder Farbempfindungen) und physikalischer Beschaffenheit von Lichtwellen (vgl. Zeki (1993): 227ff.). ${ }^{6}$ Als Alternative steht es einem Farbphysikalisten jedoch ebenso offen, nach physik-immanenten Eigenschaften der Gegenstände selbst zu suchen. Eine solche Position wird, meines Wissens nach, eigentlich nur noch von Jackson und Pargetter aufrechterhalten (vgl. Jackson \& Pargetter (1987); sowie Jackson (1996)). Auch wenn sie Farben mit - unbestimmt belassenen - mikro-physikalischen Eigenschaften gleichsetzen, wird es der nächste Abschnitt plausibel machen, ihren Ansatz derart zu interpretieren, daß sie Farben mit Reflektanzprofilen identifzieren.

\subsubsection{Farben als Reflektanzprofile}

Die eigentlich für eine physikalistische Ontologie der Farben bedeutsame Auseinandersetzung muß sich direkt auf die in Frage kommenden intrinsischen Eigenschaften von Dingen in der Außenwelt beziehen. Die empirischen Untersuchungen der physikalischen Ursachen von Farbempfindungen haben eine Vielzahl von für Farbwahrnehmungen relevanten, mikrophysikalischen Eigenschaften offenbart (vgl. Nassau (1980)). Es läßt sich jedoch zugleich ein makro-physikalisches Charakteristikum finden, welches alle farbigen Objekte gleichermaßen exemplifizieren: und zwar ihr physik-immanent bestimmbares Reflektanzvermögen oder Reflektanzprofil SSR (zur Definition dieser Eigenschaften: vgl. weiter unten im Zusammen-

6 Traditionellerweise leitet sich diese Auffassung von Newton her (vgl. Thompson (1995): 10-18); in neuerer Zeit scheint auch Armstrong - in seinen frühen Schriften über Farben - diese Position erwogen zu haben (vgl. Armstrong (1968a); vgl. Thompson (1995): $111 \mathrm{ff}$.). Doch letztere bevorzugt wohl die schwach-physikalistische Auffassung, Farben als Dispositionen anzusehen, bestimmte Lichtwellen aussenden. 
hang mit der These $\left.\left(\mathrm{PHY}_{\mathrm{STARK}}\right)\right)$. Im Prinzip scheint es relativ gleich $\mathrm{zu}$ sein, auf welcher der beiden Ebenen die Diskussion des Starken Physikalismus geführt wird. Grundsätzlich bietet sich die mikroskopische Ebene eher an, da sie sich näher an der gebräuchlichen Physik befindet; doch die Einführung der neuen und komplexen Größe der Reflektanzprofile erleichtert die Konkretisierung und Besprechung der Position erheblich. Der einzige bedeutsame Unterschied zwischen den beiden Alternativen besteht hinsichtlich der Feingradigkeit der Farbeigenschaften (und damit im Grunde auch hinsichtlich ihrer Anzahl, obwohl es in beiden Fällen zwar abzählbar, aber unendlich viele verschiedene Farben geben wird).

Gerade deswegen sollten die makroskopischen Eigenschaften eher als Farben angesehen werden. Denn für die Farberkennung durch Lebewesen ist es unwichtig, wie weit sich Farbeigenschaften theoretisch innerhalb des atomaren Bereich analysieren lassen, da ihre Diskriminierungsfähigkeit ohnehin auf die makroskopische Ebene eingeschränkt bleibt und auf dieser eine allen farbigen Objekten zukommende und physik-immanente Eigenschaft - die Reflektanzprofile - bereits identifiziert worden ist: unterhalb der Reflektanzprofile können wir keine Unterschiede in der physikalischen Beschaffenheit mehr wahrnehmen (vgl. Hilbert (1987): 100). Es ist dagegen von viel größerem Interesse, ob die betreffenden Subjekte die Genauigkeit der theoretisch vollzogenen Individuation von Farbeigenschaften praktisch nachvollziehen können oder nicht. Hinsichtlich der mikrophysikalischen Ursachen kann dies nicht der Fall sein. Dagegen ist dies für die makroskopischen Eigenschaften nicht von vorneherein ausgeschlossen, so daß die eben formulierte Frage nach der Übereinstimmung von theoretischer und sinnlicher Diskriminierungsfähigkeit hier interessant bleibt. Deswegen ist es für einen Starken Physikalismus, der eine solche Kongruenz möglichst erhalten möchte, angebrachter, sich auf die makroskopische Ebene zu konzentrieren (für jemanden, der dies nicht will, ist es ganz gleich, welche Ebene der Diskussion bevorzugt wird). Die feingradigst bestimmbaren Farben werden demnach mit den Reflektanzprofilen gleichgesetzt. Damit läßt sich also die These (PHY) bezüglich des Starken Physikalismus wie folgt konkretisieren: ${ }^{7}$

$7 \quad$ Vertreter dieser Position sind Jackson und Pargetter (1987), Jackson (1996) und Averill (1982), wobei letzterer eigentlich (aber vergeblich) versuchen möchte, einen Informationalismus zu vertreten (vgl. den entsprechenden Abschnitt), und 
(PHY $\left._{\text {STARK }}\right) \quad P:=$ Reflektanzprofile $S S R$.

Jeder einzelnen Farbe läßt sich ein konkretes, quantitativ bestimmtes Reflektanzvermögen zuordnen (und umgekehrt). Die Klasse der Farben erschöpft die Klasse der Reflektanzprofile. Zur Erinnerung sei noch einmal gesagt, daß Reflektanzprofile SSR (einschließlich des Transmittanzverhaltens, welches bestimmt, wieviel Licht ein Körper durchläßt) das Vermögen eines Gegenstandes beschreiben, hinsichtlich jeder einzelnen Wellenlänge des Lichtspektrums einen bestimmten Anteil des einfallenden Lichtes zu reflektieren (oder durchzulassen) und den Rest zu absorbieren. Theoretisch läßt sich so hinsichtlich einer gegebenen Oberfläche (oder eines Volumens) für jede Wellenlänge - und praktisch für jedes sehr schmale Wellenlängenband - ein proportionaler Wert angeben, der das Verhältnis zwischen der Intensität des einfallenden und der des reflektierten (oder durchgelassenen) Lichtes quantitativ beschreibt. Da die Intensitätsverteilung von Lichtwellen eine physik-immanente Art oder Größe darstellt, gilt dies auch für das mithilfe der Absorptionsgesetze daraus konstruierbare Reflektanzprofil von Gegenständen. ${ }^{8}$ Broackes formuliert nun aber den folgenden Einwand gegen die Sprechweise, daß Gegenstände, die uns einfarbig erscheinen, nur genau eine Farbeigenschaft haben können:

neuerdings eine subjektivistischen Dispositionalismus für richtig hält (vgl. Averill (1992). Armstrongs Position ((1993); (1997)) kann vielleicht in die Richtung eines Starken Physikalismus interpretiert werden (wie beim Vergleich von Starkem und Schwachem Physikalismus argumentiert werden soll).

8 Reflektanzprofile werden üblicherweise nicht über das gesamte Lichtspektrum, sondern nur über den von Lebewesen wahrnehmbaren Bereich (etwa von $300 \mathrm{~nm}$ bis $800 \mathrm{~nm}$ reichend) definiert werden. Doch die hier gewählte Ausweitung auf das ganze Spektrum ist vielleicht dadurch begründbar, daß einerseits außerhalb des genannten Bereiches die Wechselwirkungen zwischen Licht und Materie mit Hinsicht auf Farbphänomene vernachlässigt werden können, und daß andererseits die wahrnehmbaren Spektren in jedem Fall voneinander abweichen und somit wahrscheinlich keines mit einem subjektunabhängig gewählten Definitionsbereich des Spektrums in Deckung sein wird. Doch auch die Beschränkung auf den angegeben Bereich wird den physik-immanenten Charakter der Reflektanzprofile nicht beeinträchtigen. 
"The phrase 'the colour of the object' is indeterminate: the object may be at one and the same time red, vermillion, a highly saturated vermillion, and also R10Y 3080 (to pick a figure out of the air for the object's coordinates in the Natural Colour System). The colour of an object is like its position, which may be at one at the same time: in the house, in the bedroom, in the top drawer of the bedside table, and at such-and-such a coordinate position.“ (Broackes (1992): 455f.)

Natürlich kommen diese hierarchisch angeordneten Eigenschaften dem Gegenstand gleichermaßen zu (wie schon die Überlegungen zur hierarchischen Struktur der Farbdeterminanten gezeigt hat). Aber letztlich können all diese Farbtatsachen höherer Ordnung auf eine einzige zurückgeführt werden, auf der sie supervenieren: nämlich auf die Instantiierung der k, das heißt in diesem Fall, auf die Instantiierung eines Reflektanzprofiles. Damit hat das betreffende Objekt genau den als R10Y 3080 individuierten Farbton, und keinen anderen. Und dies allein reicht aus, um auch Zuschreibungen wie „der Gegenstand ist Zinnoberrot“" wahrzumachen. Aus ontologischer Sicht hat jeder normalerweise einfarbig erscheinende Gegenstand auch nur genau eine Farbe. Die Zuschreibungen allgemeinerer Determinanten kann durch diese Tatsache vollständig erklärt werden. Die Realität wird also nicht unnötig angereichert, da supervenierende Tatsachen laut der These des ontological free lunch kein wirkliches Mehr an Tatsachen bedeuten.

Aus zwei Gründen ist es nun jedoch nicht zu erwarten, daß die Bevorzugung der makroskopischen Reflektanzeigenschaften als Farben eine Übereinstimmung der theoretischen und der sinnlichen Diskriminationsfeingradigkeit gewährleisten kann: erstens kann die empirische Messung sich - je nach Energievorrat und technischen Möglichkeiten - beliebig genau an die Realität annähern, was den Augen aufgrund ihres vorgegeben „Auflösungsvermögen“ offensichtlich untersagt bleibt; und zweitens kann die Unbestimmtheit der Wahrnehmung nicht so ohne weiteres umgangen werden. In diesem Sinne wird unser durch Wahrnehmung erworbenes Wissen über die Realität niemals die Genauigkeit der entsprechenden, wissenschaftlichen Beschreibungen erreichen können. Es sollte also nicht erstaunen, daß die Colorimetrie zu dem Ergebnis kommt, daß viele Gegenstände mit verschiedenen Reflektanzprofilen selbst unter Normalbedingungen als Metamere gesehen werden: wobei zwei Objekte genau dann Metamere hin- 
sichtlich einer bestimmten Beleuchtung sind, wenn sie (auch trotz unterschiedlicher, physikalischer Konstitution), einem Betrachter unter dieser Beleuchtung als gleichfarbig erscheinen (vgl. Hardin (1988): 28). Der menschliche Wahrnehmungsapparat kann somit die physik-immanenten Reflektanzeigenschaften der Dinge in der Außenwelt nicht ebenso feingradig individuieren, wie es der empirischen Physik möglich ist. Dieser Umstand hat viele Vertreter eines Starken Physikalismus dazu bewogen, diesen aufzugeben und stattdessen einen Schwachen anzunehmen. Auch Hilbert ist in dem zusammen mit Byrne geschriebenen Aufsatz diesen Weg gegangen, den er in seinem früheren Buch bereits vorgezeichnet hat (vgl. Hilbert (1987): 102): ${ }^{9}$

„If we like, we can call the individual SSRs the 'maximally specific colors', although it must be stressed that this is simply a natural way of extending our everyday color talk. The reflectance-types that the human visual system represents objects as having are considerably coarser than the maximally specific colors. Hence, although of course object having maximally specific colors are visible, the maximally specific colors themselves are not, because they are not properties that one can tell an object possesses simply by looking at it. That is why the terminology is an extension of ordinary usage. [...] A color property is simply a reflectance-type.“ (Byrne \& Hilbert (1997c): 266)

Eine erste Konsequenz, die sich aus dem Phänomen der Metamerik unter Normalbedingungen ergibt, ist, daß nicht alle Farbtatsachen und Farbunterschiede (von uns oder anderen Lebewesen) wahrgenommen oder repräsentiert werden können. Das heißt aber nichts anderes, als daß die These $\left(\mathrm{IND}_{\mathrm{R}}\right)$ nicht alle Farbeigenschaften erfassen kann. Es stellt sich zudem die berechtigte Frage, ob darüberhinaus nicht ganz allgemein die Repräsentierbarkeit der Farben verletzt ist. Denn das Auftreten des Metamerismus im-

9 In seinem Buch hat Hilbert die Reflektanzprofile noch als „maximal bestimmte Farben“, ,individuelle Farben“ oder einfach „Farben“ bezeichnet, ohne genügend auf den Umstand hinzuweisen, daß diese „Farben“ nicht wahrnehmbar sind, sondern nur die mit Typen oder Klassen von Reflektanzprofilen identifizierten Farben. Kritik hat Hilbert dazu bewogen, diese Bezeichnungsweise aufzugeben, so daß im neueren Aufsatz nun nur die Reflektanztypen als ,maximal bestimmte Farben“" angesehen werden (vgl. Byrne \& Hilbert (1997c): 276; sowie Fn. 12). Doch dazu - und zu dem Begriff des Reflektanztypes - mehr im Abschnitt über den Schwachen Physikalismus. 
pliziert ja, daß unter Normalbedingungen unsere Farbempfindungstypen nicht jeweils mit einzelnen Reflektanzprofilen nomologisch korreliert sind; während die Repräsentationsrelation gerade als nomologische Korrelation unter Normalbedingungen zu verstehen ist. Die einzig mögliche Schlußfolgerung ist also anscheinend, daß unsere Farbwahrnehmungen keine einzelnen Reflektanzprofile repräsentieren können, sondern höchstens bestimmte Mengen von Reflektanzprofilen. Der Schwache Physikalismus (wie der von Byrne und Hilbert vertretene) zieht hieraus die Konsequenz, Farben mit Disjunktionen oder Dispositionen gleichzusetzen. Der Primitivismus löst das Problem, indem er Farben als primitive, nicht weiter bestimmbare Eigenschaften ansieht. Wie es scheint, wird der Starke Physikalismus hingegen erzwungenermaßen akzeptieren müssen, daß Farben faktisch keine (von uns) repräsentierten oder repräsentierbaren Eigenschaften sein können: eine Ansicht, die anscheinend das gesamte Fundament der physikalistischen Position - nämlich die Thesen $\left(\mathrm{IND}_{\mathrm{R}}\right)$ und (REP) - untergräbt.

Hierin liegt ein wirkliches Dilemma für den Starken Physikalisten verborgen. Ohne diese Grundannahme besteht erst einmal gar kein Grund, Reflektanzprofile als Farben anzusehen - und nicht eben bloß als Reflektanzprofile. Denn wenn letztere weder von unseren Farbwahrnehmungen repräsentiert werden, noch irgendeinen Bezug zu deren phänomenalen Aspekt aufweisen (welcher nur durch die Repräsentationalität vermittelt sein kann, da Qualia unabhängig vom Gehalt keinen Weltbezug aufweisen können), besteht für uns gar kein Anlaß, die Reflektanzprofile als Farben zu betrachten: die beiden Thesen (REP) und (PHE) wären nicht erfüllt. Es wird dann zudem viel plausibler, die erwähnten Klassen von Reflektanzprofilen (oder die Reflektanztypen), welche unter Normalbedingungen sehr wohl eine nomologische Korrelation zu unseren Farbsinneserfahrungen zeigen, mit Farben gleichzusetzen. Damit wird aber der Bereich des Starken Physikalismus verlassen und der des Schwachen betreten, weil Reflektanztypen oder Klassen von Reflektanzprofilen, wie noch gezeigt werden soll, keine physikalische Art darstellen. Der Starke Physikalist steht also vor der Alternative, entweder seine Position ganz aufzugeben und zur schwächeren Variante des Farbphysikalismus überzuwechseln, oder aber mit dem Vorwurf leben zu müssen, eine Theorie der Reflektanzprofile, aber nicht der Farben, zu vertreten. Gerade der letztere Punkt sollte noch einmal herausgehoben werden: was zur Debatte steht, ist die Natur der Farben; die Natur 
der Reflektanzprofile ist im großen und ganzen unkontrovers. Hardin kommentiert die Möglichkeit, die Repräsentiertheit aufzugeben, folgendermaßen:

\begin{abstract}
„As physical candidates for identification with the colors, we have so far considered: detailed physical [i.e., microphysical - der Autor] mechanisms...; higherlevel properties of matter, such as reflectance; and the basic characteristics of light, particularly wavelength. All of these are causally important to the perception of color, but none of them can serve as a basis for sorting visible objects into the color classes that we and like creatures employ. We may, of coure, decide to settle for such properties as relative spectral reflectance, illuminance, etc. [» reflectance profiles $S S R$ - der Autor] as constituting 'physical color', and drop the requirement that objects that match metamerically over a wide range of illuminants are to be denominated as having the same color. However attractive this strategy may seem on other grounds, one must realize that the concept of 'color' it yields is one in which two matching yellow spots will usually not have the same color. If one wants an account of the ontological locus of red, green, yellow, and blue - what features they share, and in what ways they differ - one must look elsewhere. And it may fairly be said that it is these questions which have excited the attention of philosophers.“ (Hardin (1988): 64f.)
\end{abstract}

Ob die Konsequenzen für einen Starken Physikalisten wirklich so weitreichend sind, wie es Hardin beschreibt, wird noch zu klären sein. In jedem Fall wird es ersteren einige Anstrengungen kosten, diese Schwierigkeit für seine Theorie der Farben zu bewältigen. Betrachten wir jedoch zunächst die Ursache für das Dilemma - oder besser gesagt: für die Metamerik von Reflektanzprofilen unter Normalbedingungen - etwas genauer. Im großen und ganzen lassen sich hierfür zwei Gründe benennen, welche sich beide auf Einschränkungen der Rezeptoren im Auge zurückführen lassen. Erstens gibt es nur drei Rezeptorarten, so daß das Auge nur für drei Wellenlängenbereiche sensitiv ist, und nicht etwa für jede einzelne Wellenlänge; damit geht bereits eine enorme Menge von spezifischen Informationen über das einfallende Licht verloren, oder wird eingeebnet. Und zweitens ist die Empfindlichkeit der Zäpfchen nur auf einen engen Bereich des Lichtspektrums begrenzt: nämlich das wahrnehmbare Spektrum; die Beschaffenheit der Reflektanzprofile in den darüber hinausragenden Bereichen des gesamten Lichtspektrums wird somit für das menschliche Farbensehen irrelevant.

Es handelt sich dabei jedoch um ein generelles Problem für alle wahr- 
nehmenden Subjekte und alle wahrnehmbaren Tatsachen. Faßt man Sinnesorgane als eine Art von Meßinstrumenten auf, so wird ganz klar: daß es zum einen immer empirische Methoden geben wird, mit denen bestimmte Tatsachen mit größerer Detailgenauigkeit gemessen werden können als mit unseren Sinnen, und daß der Grad an Genauigkeit oder Feingradigkeit der Messung im Prinzip beliebig hoch liegen kann; und daß zum anderen die Sinnesorgane, genauso wie alle Meßinstrumente, zwangsläufig einer gewissen Ungenauigkeit unterliegen. So ist es möglich, zwei Strecken als gleichlang zu sehen, obwohl eine Messung unterhalb des Millimeterbereiches eine leichte Längendifferenz feststellt; und zwei andere Stäbe, die wiederum vom letzteren Meßinstrument die gleiche Länge zugewiesen bekommen, können sich im Mikrometerbereich unterscheiden (und so weiter). Weder eine visuelle Sinneserfahrung, noch ein Meßprozeß können zu einem exakten Ergebnis kommen: auch wenn der Stab $10 \mathrm{~cm}$ lang ist, kann er höchstens als in etwa $10 \mathrm{~cm}$ lang gesehen oder gemessen werden. In diesem Sinne ist es also gar nicht überraschend, daß unser Auge Reflektanzprofile nicht exakt diskriminieren kann: nicht zuletzt auch deswegen, da diese Reflektanzen über die Physik analysiert werden. Auch Hilbert diskutiert diese Schwierigkeit, welche er als eine Unbestimmtheit der Wahrnehmung bezeichnet (vgl. zu diesem Thema Hilberts Erläuterungen in (1987): 103-109).

„The indeterminacy of color perception with respect to the underlying physical facts is not unique to color perception. All perception is indeterminate as well as all all measurement. [...] Our perceptions of length do place constraints on the range within which the determinate length of the perceived object can lie. This sort of indeterminacy is also familiar to us from measurements of physical quantities which are usually reported with the margin of error attached. [...] The exact range of lengths compatible with a particular perception of length will be partly a function of the viewing context, i.e., distance from perceiver, orientation, and lightning, and partly a function of the characteristics of the visual system of the perceiver. The perception of color does not differ in this respect from the perception of length.“ (Hilbert (1987): 102)

Der Grund für diese Unbestimmtheit liegt in dem endlichen Auflösungsvermögen von Meßgeräten, das heißt ganz allgemein: an dem Vermögen, Unterschiede in der Quantität einer gegebenen Größe feststellen zu kön- 
nen. Ein Lineal mit einer Millimetereinteilung kann Längen eben nicht genauer als auf einen Millimeter bestimmen; normale Mikroskope gelangen in den Mikrometerbereich, während Elektronen- oder Feldemissionsmikroskope sogar ein Auflösevermögen bis hin zum Umfang von Atomen zeigen. Zudem sollte darauf hingewiesen werden, daß die Unbestimmtheit der Wahrnehmung durchaus ihre biologische Funktion hat. Es ist beispielsweise für Menschen wenig sinnvoll, mikroskopische Unterscheidungen treffen zu können, wenn wir doch selbst makroskopische Objekte sind und uns in einer makroskopischen Welt bewegen. Während unser Auge, so wie es sich entwickelt hat, seine biologische Funktion ganz gut erfüllen kann, hätte die Enwicklung und der Besitz zusätzlicher und feingradigerer visuelle Sinnesorgane nur einen Energieverbrauch bedeutet, der durch den Gewinn an Information bei weitem nicht aufgewogen worden wäre: ein bedeutsamer evolutionärer Vorteil hätte damit dann nicht bestanden. Ein sehr ähnliches Problem ist bereits bei der Bestimmung der Normalbedingungen aufgetreten, da Subjekte hinsichtlich der Wahrnehmung von Eigenschaften zweiter Ordnung von Farb- und Formeigenschaften mehr oder weniger leicht voneinander abweichen können; auch dort war die Unbestimmtheit der Wahrnehmung für das Problem verantwortlich.

Hilbert zieht nun aus der Unbestimmtheit der Wahrnehmung die oben bereits angedeutete Konsequenz, daß nicht einzelne Reflektanzprofile, sondern Klassen von Reflektanzprofilen wahrgenommen werden: wie auch nicht einzelne, exakte Längen, sondern Längen innerhalb eines bestimmten, groben Intervalles gesehen werden. Diese wahrnehmbaren Klassen von Eigenschaften unterliegen dabei nicht mehr der Unbestimmtheit der Wahrnehmung, weil sie gerade so definiert werden, daß sie diese Unbestimmheit und die resultierende Metamerik umgehen. In jedem Fall wird sowohl die Wahrnehmung der primären Qualitäten als auch die der sekundären diese Unbestimmtheit aufweisen: und zwar beidesmal aufgrund der Einschränkungen, die durch die Wahrnehmungssituation und, vor allem, durch die Sensitivität der Sinnesorgane vorgegeben sind. Es werden nicht einzelne physikalische Eigenschaften, sondern Klassen oder Typen von Eigenschaften wahrgenommen. Dies gilt nebenbei auch für die - weitaus größere - Unbestimmtheit, die Farb- oder Formausdrücke zeigen, welche sich ebenfalls nicht auf einzelne Eigenschaften in der Welt, sondern auf Eigenschaftsgruppen beziehen (aber auch diesbezüglich scheint es keinen kate- 
gorialen Unterschied zwischen primären und sekundären Eigenschaften zu geben).

„Surface spectral reflectances are the maximally determinate colors. Our colour language is less determinate than our perceptions of color, but not even our color perception correspond to completely determinate colors. [...] What we need to realize in order to avoid these problems is that neither perception nor language are perfectly determinate. In both perception and language we are given kinds of colors, not maximally determinate colors themselves." (Hilbert (1987): 102; Hervorhebungen des Autors)

Es besteht jedoch ein bedeutsamer Unterschied zwischen Farb- und Längenwahrnehmung hinsichtlich der physikalischen Beschaffenheit der wahrgenommenen Klassen, der auf gewisse Weise dem in der Einleitung erwähnten Gedanken Lockes entspricht, daß nur unsere Vorstellungen oder Wahrnehmungen von primären Qualitäten diesen auch in gewisser Weise ähnlich sind. Während als gleichlang gesehene Stäbe auch aus physikalischer Sicht in etwa dieselbe Länge aufweisen, können zwei als gleichfarbig empfundene Gegenstände physikalisch betrachtet ganz unterschiedliche Reflektanzprofile aufweisen. Wird ein Stab zutreffenderweise als in etwa $10 \mathrm{~cm}$ lang gesehen, so kann man sicher sein, daß empirisches Nachmessen die Länge des Stabes in einem recht schmalen Intervall um die $10 \mathrm{~cm}$ herum verorten wird: trotz statistischer Fehlerhaftigkeit wird es keine allzugroßen Abweichungen geben (zum Beispiel nicht mehr als einen halben oder einen ganzen Zentimeter). Dagegen weisen metamerische Gegenstände meistens ganz unterschiedliche spektrale Oberflächenreflektanzen (das heißt: Reflektanzprofile) auf: während die eine sich vielleicht durch eine recht flache und gleichmäßige Reflektanzkurve darstellen läßt, kann die andere in ihrer Kurve ohne weiteres sehr viele und extreme Minima und Maxima aufweisen (vgl. Hardin (1988): 47; sowie Hilbert (1987): 103). Es gibt bei Farben also keine „Intervalle“, die die mögliche Abweichung von einem durchschnittlichen Reflektanzprofil angeben könnten.

Das Auge funktioniert sozusagen nur hinsichtlich der Detektion von Längen - nicht aber hinsichtlich der Erkennung von Reflektanzdifferenzen - wie ein Meßinstrument in der Physik. Es gruppiert zwar Längen auf vergleichbare Weise in Klassen, wie es auch ein Längenmeßgerät in der Phy- 
sik mit ähnlicher Sensitivität tun würde; aber ein physikalisches Meßinstrument für die quantitative Bestimmung von Reflektanzprofilen wird diese ganz anders in Mengen zusammenfassen, als es unser visuelles System macht. Denn ersteres wird die beiden eben beschriebenen Reflektanzkurven als sehr unterschiedlich ansehen, während sie in uns dieselbe Farbempfindung hervorrufen. Auch wenn Hilbert zu Recht auf die Möglichkeit hinweist, Instrumente zu bauen, die Reflektanzprofile mit derselben Sensitivität und Unbestimmtheit wie unser visuelles System detektieren können, wird es sich hierbei nicht mehr um Meßgeräte handeln, die eine physikalische Größe quantitativ bestimmen können. Denn es kann hier gar nicht mehr von einem Auflösungsvermögen hinsichtlich der Unterscheidung von spektralen Oberflächenreflektanzen gesprochen werden, da eine zugrundezulegende, physikalische Maßeinheit fehlt. Während Reflektanzprofile als physikalische Eigenschaft, je nach Auflösungsvermögen der betreffenden, physikalischen Meßgeräte, in mehr oder weniger breiten Intervallen zusammengefaßt werden können, spielt die Klasse der Reflektanzprofile, die wir als Farbmetamere sehen, als solche für die Physik keine Rolle. Zwar sind ihre Mitglieder physikalische Eigenschaften, doch die Klasse selbst stellt keine physikalische Art dar (diese letzte These wird erst im Abschniit über den. Schwachen Physikalismus begründet).

Dies weist bereits daraufhin, daß diese Klassen oder Typen von Reflektanzprofilen nicht als physik-immanente Eigenschaften angesehen werden können, sondern nur als (wahrscheinlich subjekt-relative) Eigenschaften zweiter Ordnung. Hilbert bewegt sich hier also schon auf einen Schwachen Physikalismus zu. Davon ganz abgesehen zeigt sich in den letzten Ausführungen nun doch ein wesentlicher Unterschied zwischen der Wahrnehmung von den sogenannten primären und sekundären Qualitäten (der so von Hilbert zwar gesehen, aber nicht betont wird). Die von uns wahrgenommenen Klassen von physikalisch meßbaren Längeneigenschaften bilden eine physikalische Größe; unser visuelles System funktioniert wie ein physikalisches Längenmeßinstrument mit einem auf makroskopische Objekte beschränkten Auflösungsvermögen (und natürlich ohne eine auf Metern als Grundeinheit basierender Skalierung). Dagegen stellen Klassen von Reflektanzprofilen, wie sie von unseren Farbwahrnehmungen zusammengestellt werden, keine physikalische Art dar; die einzelnen Klassen oder Ty- 
pen der metamerischen Reflektanzprofile sind, obwohl physikalisch beschreibbar, nicht physik-immanente Entitäten, sondern müssen anderweitig individuiert sein. Auch Hilbert kommt zu diesem Schluß, wobei er eindeutig Wert auf die den primären und sekundären Qualitäten gemeinsame Objektivität der Analysierbarkeit legt:

„The fact that the relation between perceived color and determinate color is more complex than the relation between perceived length and determinate length is no argument that the one quality is more subjective than the other. In both, cases one can give a description of the class of determinate properties that is consistent with any given perception that makes no reference to subjective characteristics of the percept. Although the classes that our perceptions of color sort reflectances into are determined by the peculiar characteristics of our color vision apparatus, it is possible to specify these classes in a perfectly objective manner. [...] Thus although there is no physical property tying together all objects that appear to have a particular length [or color - der Autor] there is an objectively specifiable, albeit anthropocentric, property that such objects share." (Hilbert (1987): 109ff.)

In dem letzten Satz des Zitates wird ganz deutlich, daß es sich bei dieser Auffassung nicht mehr um einen Starken Physikalismus handelt. Das den Farben gemeinsame Merkmal $P$ und die mit den konkreten Farben identifizierten Eigenschaften $P_{\mathrm{i}}$ sind zwar physikalisch beschreibbar, aber sie haben ihre physikalische Beschaffenheit nicht wesentlich als Farben: die $P$ Eigenschaften sind nicht physik-immanent. ${ }^{10}$ Das Zitat deutet daraufhin, daß Hilbert diese Meinung auch in Hinsicht auf Längen vertritt: die vom visuellen Apparatus detektierten Klassen von Eigenschaften sind nicht physik-immanent. Das kann aber nicht sein, wenn das Auge hinsichtlich von Längen als physikalisches Meßinstrument angesehen werden kann: was plausibel ist vor dem Hintergrund, daß physikalische Meßgeräte mit demselben Auflösungsvermögen wie das Auge Längen genauso in Intervalle zusammenfassen wie unser visuelles System (mit Bezug auf Farben ist ein solches physik-relevantes Meßinstrument nicht möglich). Demnach sind die von uns wahrgenommenen Klassen von Längeneigenschaften genauso physik-immanent wie die von einem physikalischen Meßgerät gemessenen

10 Es sollte beachtet werden, daß Hilbert, wenn er hier von physikalischen Eigenschaften spricht, das meint, was ich als physik-immanente Eigenschaften bezeichne (vgl. Hilbert (1987): 100). 
Klassen. Für die Klassen der metamerischen Reflektanzprofile gilt dies aber nicht.

Auch wenn die hier plausibel gemachte These - daß es sich hierbei aufgrund der fehlenden Physik-Immanenz nicht mehr um einen Starken Farphysikalismus handeln kann - noch im nächsten Abschnitt über den Schwachen Physikalismus argumentativ gestützt werden muß, sollte schon jetzt (ihre Gültigkeit vorausgesetzt) klar sein, daß der Starke Physikalist mit Bezug auf Farben nicht Hilberts Weg gehen und die Unbestimmtheit von Farbwahrnehmungen auf Repräsentation von Klassen von Reflektanzprofilen zurückzuführen kann. Es bleibt also nur die andere Alternative des Dilemmas übrig: daß Reflektanzprofile als Farben identifiziert werden, ohne daß diese Identifikation durch einen angemessenen Zusammenhang zwischen spektralen Oberflächenreflektanzen und unseren Farbwahrnehmungen begründet werden kann. Man könnte zugegebenermaßen sagen, daß Reflektanzprofile als Farben gelten sollten, weil wir Klassen solcher Eigenschaften als Farben repräsentieren. Aber dann wäre es von vorne herein angebrachter, diese Klassen - und nicht ihre Mitglieder - als Farben anzusehen. Denn die entgegengesetzte These, die von einem Starken Physikalisten dann eigentlich angestrebt werden müßte, ist nicht möglich: daß wir Klassen von Reflektanzprofilen als Farben wahrnehmen, weil die letzteren, physik-immanenten Eigenschaften tatsächlich Farben sind. Hierfür bedürfte es zunächst einer davon unabhängigen Begründung oder Motivation für die Identifikation von Farben mit Reflektanzprofilen.

Die Asymmetrie zwischen Farben und Formen hat sich bisher in den Alternativen für einen Farbphysikalisten ausgedrückt, entweder auf die Wahrnehmbarkeit oder Repräsentierbarkeit von Farben zu verzichten, oder aber auf deren Physik-Immanenz. Daß dieser Zwang zur Auswahl besteht, deutet dagegen auf einen fundamentalen Unterschied zwischen Farben und Formen hin, da es hinsichtlich letzterer - trotz der Unbestimmtheit der Wahrnehmung - unproblematisch ist, sowohl die Physik-Immanenz als auch die Repräsentierbarkeit anzunehmen: der Formphysikalist kann beide Merkmale aufrechterhalten, weil die repräsentierten Klassen von physikimmanenten Längeneigenschaften wiederum physik-immanent sind. Damit ist - im Gegensatz zu der im Zusammenhang von Normalbedingungen gezogenen Schlußfolgerung - eine Differenz zwischen primären und sekundären Qualitäten gefunden worden: die jedoch nicht auf der Unbestimmt 
der Wahrnehmung, sondern auf Unterschieden in der wahrnehmbaren Natur der betreffenden Eigenschaften beruht. Denn wir können zwar physikimmanente Längeneigenschaften wahrnehmen, aber keine physik-immanenten Reflektanzcharakteristika. Die Sensitivität des Auges ist hinsichtlich von Formen nur in der Ungenauigkeit, hinsichtlich von Reflektanzprofilen jedoch auch in der Erfassung der physikalisch beschreibbaren Anordnung begrenzt (deswegen scheint ein Formobjektivist auch mit dem Ausdruck „Längen“ auszukommen, während der Farbobjektivist die beiden Ausdrücke „Farben“ und „Reflektanzprofile“ benötigt).

Doch es gibt für den Farbphysikalist wenigstens noch einen dritten Weg, der der Unbestimmtheit der Wahrnehmung Rechnung trägt und gleichzeitig die Aufrechterhaltung eines Starken Physikalismus zu ermöglichen scheint. Denn es kann versucht werden, die Reflektanzprofile als die von Farbwahrnehmungen repräsentierten Eigenschaften aufrechtzuerhalten, indem nicht die nomologischen Korrelationen zwischen den Reflektanztypen und den Qualia der Wahrnehmungstypen als entscheidend für die Repräsentationalität angesehen werden, sondern die diesen zugrundeliegenden, kausalen Gesetzmäßigkeiten, welche zwischen den Reflektanzprofilen und feingradiger bestimmten Wahrnehmungstypen bestehen. Demnach können Farbwahrnehmungen nur noch über ihren Gehalt, nicht aber über ihr Quale typen-individuiert werden. Ein einfaches Beispiel von Averill kann das Problem erläutern helfen (vgl. Averill (1982): 12ff.). ${ }^{11}$ Gegeben ist ein Bild mit einer Figur vor einem Hintergrund: die Figur und die Hintergrundsfläche verfügen zwar über unterschiedliche Reflektanzprofile, sind aber beide unter Normalbedingungen metamerisch. Eine der beiden folgenden Aussagen über das Bild muß nun falsch sein: entweder haben die Figur und der Hintergrund verschiedene Farben, da sie verschiedene Reflektanzprofile aufweisen; oder aber sie haben dieselbe Farbe, da Farb gehalt und Farbqualia gleichermaßen feingradig spezifiziert sind und die repräsentierten Farben unter Normalbedingungen über die Phänomenalität

11 Averill formuliert das Beispiel als ein Trilemma, welches er für einen Schwachen Physikalisten (hier: Smart) als nicht so einfach zu lösen ansieht. Sein Einwand gegen Smart ist für diesen jedoch nicht problematisch, da das Trilemma nur auf einem Benennungsfehler von Averill beruht (er unterscheidet zwischen Farben und Farbtönen, müßte aber eigentlich zwischen realer und erscheinender Farbe differenzieren). 
der Wahrnehmungen individuiert werden. Dabei handelt es sich genau um die Optionen eines Starken und eines Schwachen Physikalismus.

Averill bevorzugt die erstere Alternative, auch wenn die These (ACT${ }_{\mathrm{NB}}$ ), wie sein Beispiel zeigt, dann nicht mehr gültig sein kann. Denn es ist nicht mehr - oder nur noch sehr grob - möglich, Gehaltsunterschiede introspektiv festzustellen. Zwar ist auch weiterhin mit jeder phänomenalen Differenz ein Unterschied in der repräsentierten Eigenschaft gegeben, aber umgekehrt gilt dies nicht mehr. Eine Sinneserfahrung mit einem Rotquale und eine mit einem Blauquale repräsentieren beispielsweise immer noch zwei verschiedene Reflektanzprofile; doch dies ist aller Wahrscheinlichkeit ebenso der Fall, wenn zwei rot-phänomenale Wahrnehmungen vorliegen, da es unzählig viele Reflektanzprofile gibt, die in einem Betrachter unter Normalbedingungen introspektiv identische Farbwahrnehmungen hervorrufen können. Um den genauen Typ einer gegeben Farbwahrnehmung zu bestimmen, ist es demnach erforderlich, empirische Forschungen mit in Betracht $\mathrm{zu}$ ziehen. Und in jedem Fall gilt die Aktualitätsthese $\left(\mathrm{ACT}_{\mathrm{NB}}\right)$ nicht mehr. Sie muß durch die schwächere These ersetzt werden, daß faktisch nur noch bestimmte Klassen von repräsentierten Eigenschaften mit den Farbqualia bijektiv korreliert sind. Auch Averill kommt zu dieser Konsequenz (wobei er Farben als Reflektanzprofile über deren Vermögen, Lichtwellen auf besondere Weise zu beeinflussen, individuiert):

„If the preceding analysis is right, the distinctions we make between color-sets and between shades go only as deep as the unaided eye can go. This is unlike the distinctions we draw between colors, which go as deep as many of of the distinctions between lightwaves. Although the distinctions between color-sets are not so deep as the distinctions between colors, they are for that very reason easier to apply and more suitable in the everyday identification of objects." (Averill (1982): 30)

Ein sehr ähnliches Problem stellt sich für Externalisten hinsichtlich des Gehaltes von Meinungen über natürliche Arten. Auch wenn der weite Gehalt eines auf der Erde gehabten Glaubens über Wasser (mit der chemikalischen Struktur $\mathrm{H}_{2} \mathrm{O}$ ) sich von dem eines auf der Zwillingserde gehabten Glaubens über Twasser (mit der Struktur $X Y Z$ ) deutlich unterscheidet, kann ein Subjekt, welches von der Erde auf die Zwillingserde transportiert wird (oder 
umgekehrt) diese Differenz introspektiv nicht ausmachen. Externalisten, die zusätzlich auch einen engen Gehalt zulassen, nehmen an, daß nur dessen Veränderungen durch Introspektion erfaßt werden können. Doch sie lösen das Problem damit ebenso wenig wie die Anhänger eines reinen Externalismus, welche enge Gehalte von vorne herein ablehnen. Denn in beiden Fällen bleiben nicht alle Unterschiede im weiten Gehalt (der dem allgemeinen Gehalt von Wahrnehmungen entspricht) introspektierbar (vgl. dazu zum Beispiel die Diskussionen in Burge (1979) undBoghossian (1990)).

Doch mit Bezug auf die Farbproblematik zeigt sich die zusätzliche Schwierigkeit, daß es hier um nicht-introspektierbare Gehaltsdifferenzen innerhalb der aktualen Welt geht. Deswegen steht die Alternative eines reinen Externalismus hinsichtlich des Wahrnehmungsgehaltes - wie ihn der Repräsentationalismus mit sich bringen würde - für einen Starken Farb physikalismus gar nicht offen, da dieser eine notwendige Verbindung zwischen Gehalt und Quale annimmt (dieses Problem kann sich auch für die Meinungen über natürliche Arten stellen, wenn von möglichen Welten ausgegangen wird, in denen sowohl $\mathrm{H}_{2} \mathrm{O}$ als auch $X Y Z$ vorkommt und beides in uns Überzeugungen über Wasser hervorrufen würde). Ein physikalistischer Objektivismus $\mathrm{NEC}_{\mathrm{N}}$, der die These (NEC) annimmt, ist nicht damit vereinbar, daß der repräsentationale Gehalt feingradiger bestimmt wird als das Quale - es sei denn, die Notwendigkeitsthese wird derart eingeschränkt, daß die Sensitivität der Rezeptoren die Untergrenze für die zu betrachtenden Gehaltsunterschiede angibt, was jedoch nicht im eigentlichen Sinne des Repräsentationalismus sein kann. Es bleibt also nur die Alternative des Objektivismus $_{\mathrm{ACT}}$, will man den (nicht-primitiven) Starken Physikalismus aufrechterhalten. Dieser wählt eine ähnliche Strategie wie der Theoretiker, der für Meinungen einen weiten und einen engen Gehalt akzeptiert: während Qualiadifferenzen im Normalfall immer introspektiv zugänglich sind, gilt dies für Gehaltsunterschiede streng genommen nicht. In der Praxis mag dies nicht allzu sehr stören, da das Auge die ihm vorenthaltene Information in der Regel nicht benötigt (und sonst auf die Empirie zurückgegriffen werden kann, wenn größere Genauigkeit gefragt ist). Doch theoretisch ist diese Lösung nicht sehr zufriedenstellend.

Zudem gibt es ein zweites Problem, welches vielleicht jedoch nicht ganz so schwer wiegen wird: es liegen auf einmal zwei nomologische Kor- 
relationen hinsichtlich einer gegebenen Wahrnehmung vor. Diese Verdoppelung läßt sich darauf zurückführen, daß es Sinneserfahrungen nun auch auf zwei verschiedene Weisen typen-individuiert werden können: introspektiv über ihr Quale, und empirisch über ihren Gehalt. Werden also auch zwei Eigenschaften - einmal ein Reflektanzprofil und einmal ein Reflektanztyp - repräsentiert? Der Starke Physikalist wird dies zu leugnen versuchen. Einerseits kann er argumentieren, daß Reflektanztypen gar keine genuinen Eigenschaften sind, insbesondere da es sich dabei um nicht-physikimmanente Klassen handelt. Und andererseits kann er davon ausgehen, daß Wahrnehmungszustände immer nur von einem einzigen Typus sein können, und zwar vielleicht aufgrund der feingradigeren, empirischen Individuierungsmöglichkeit. Doch diese Annahme mutet nicht nur willkürlich an, sie ist wahrscheinlich auch gar nicht durchführbar. Denn selbst wenn motiviert werden kann, daß nur die Farben, als Reflektanzprofile verstanden, repräsentiert werden, eröffnen sich zwei weitere, entscheidende Schwierigkeiten für den Starken Physikalismus.

Erstens ergibt sich, wie gesagt, das Resultat, daß die meisten Gegenstände ganz einfach unterschiedliche Farben besitzen, auch wenn wir die meisten dieser Unterschiede nicht bemerken. Die Farbigkeit der Objekte in der Welt kann dann aber nicht mehr unser auf Farbwahrnehmungen begründetes Verhalten erklären: warum wir etwa Gegenstände in Farbklassen einteilen oder auch Gegenstände aufgrund ihrer uns erscheinenden Farbe individuieren und wiedererkennen können. Um dies zu tun, muß der Starke Physikalist doch wieder auf die mit der Farbphänomenalität eng verbundenen Klassen von Reflektanzprofilen zurückgreifen. Denn der Umfang unserer Farbbegriffe entspricht nicht der Feingradigkeit von einzelnen Reflektanzprofilen, sondern der von diesen Klassen. Und zweitens können die Normalbedingungen für Farbwahrnehmungen ebenfalls sinnvollerweise nur über solche Typen von Reflektanzeigenschaften bestimmt werden, da die Reflektanzprofile von uns - ganz gleich, unter welchen Bedingungen sinnlich niemals genau diskriminiert werden können. Es ist auch gar nicht die biologisch evolvierte Funktion des Auges, solche feingradig spezifizierten Eigenschaften zu repräsentieren. Damit aber eine Repräsentation von Reflektanzprofilen möglich sein kann, sollten Normalbedingungen formulierbar sein, um entsprechend eine nomologische Korrelation aufstellen zu können. Doch wie kann diese Forderung angemessen erfüllt werden? 
Der Starke Physikalist bleibt eine Antwort auf diese Frage - wie auch eine Lösung für das oben beschriebene Dilemma - schuldig. Entweder muß er die Repräsentierbarkeit und die Wahrnehmbarkeit von Farben aufgeben, oder von nicht introspektierbaren Gehaltsunterschieden ausgehen. Die letztere Alternative scheint dabei - trotz des Verdoppelungsproblemes - noch um einiges plausibler zu sein; doch auch sie hat, wie eben beschrieben, wesentliche Mängel. Zudem steht für einen physikalistischen Notwendigkeitsobjektivismus wahrscheinlich sogar keiner dieser beiden Auswege offen. Averill zieht hieraus die Konsequenz, die Repräsentationalität durch eine Informationalität zu ersetzen. Als Alternative stehen natürlich der Primitivismus und der Schwache Physikalismus zur Auswahl bereit. Bevor nun die letztgenannte Position skiziziert werden soll, lohnt ein kurzer Blick auf den Informationalismus, wie er in Texten von Averill (1982) und Tolliver (1994) vorgeschlagen und ausformuliert wird (wobei Tolliver im Grunde keine objektivistische Position mehr vertritt).

\subsubsection{Informationalismus}

Im folgenden soll die Möglichkeit bewertet werden, einen Objektivismus von der Annahme der Repräsentationalität der Farbwahrnehmungen zu trennen und von diesen als informationelle Zustände auszugehen. Das heißt, diese Sinneserfahrungen sind unabhängig davon, unter welchen Umständen sie auftreten, immer als korrekt anzusehen. Mögliche Fehler in der Repräsentation werden dann erst auf der begrifflichen, beurteilenden Ebene auftreten können. Die Motivation für Averill, von der Repräsentationalität abzusehen, liegt in zwei Umständen begründet. Erstens bringt der Starke Physikalismus es mit sich, daß unter Normalbedingungen der Gehalt einer Wahrnehmung feingradiger als ihr Quale spezifiziert werden muß. Und daß Averill die Aufgabe der Aktualitätsthese unter Normalbedingungen akzeptiert, ist bereits gesagt worden. Zweitens lehnt er jedoch überdies die Möglichkeit ab, Farben als physikalische Arten anzusehen, die manchmal richtig und manchmal falsch wahrgenommen werden. Doch seine Argumentation hiergegen ist nicht überzeugend. Averill beginnt mit der Feststellung, daß laut Kripke die Extension von Farbausdrücken vermittels paradigmatischer Fälle von Farbwahrnehmungen unter Normalbedingungen fixiert 
wird:

„The reference of 'yellowness' is fixed by the description 'that (manifest) property of objects which causes them, under normal circumstances, to be seen as yellow (i.e., to be sensed by certain visual impressions)' “" (Kripke (1972): 140 Fn. 71)

Es entsteht ein offensichtliches Problem für diese Fixierung aufgrund der Disjunktivität von den normalen Ursachen für Farbwahrnehmungen. Wenn man erneut das obige Beispiel der Figur vor dem Hintergrund betrachtet, die beide unter Normalbedingungen gleichfarbig erscheinen, aber verschiedene Reflektanzprofile zeigen, dann wird die Schwierigkeit deutlich. Averills Argument verläuft in zwei Schritten. Es ist zum einen nicht möglich, der Figur und dem Hintergrund dieselbe Farbe - sagen wir: Gelb - zuzuschreiben und gleichzeitig davon auszugehen, Farben als physikalische Arten zu betrachten, weil disjunktive Eigenschaften keine solchen Arten sein können (wie der Abschnitt über den Schwachen Physikalismus bestätigen wird). Und zum anderen macht es auch wenig Sinn, die Farbe Gelb mit, sagen wir, dem Reflektanzprofil der Figur zu identifizieren (welches natürlich eine physikalische Art darstellen würde), indem diese - wie im Zitat beschrieben - als Paradigma für Gelbwahrnehmungen unter Normalbedingungen herangezogen wird; denn dann würden unsere meisten Gelburteile oder -wahrnehmungen falsch sein, da nur sehr wenige Gegenstände, die wir als gelb sehen, exakt dasselbe, gelb-paradigmatische Reflektanzprofil aufweisen werden wie die Figur. Somit schließt Averill, daß Farben nicht auf diese Weise als physikalische Arten angesehen werden können.

Diese Schlußfolgerung ist natürlich richtig. Doch es gibt natürlich noch die Möglichkeit, daß es viele verschiedene Farbeigenschaften gibt, die von uns normalerweise als gelb gesehen werden: wenn beispielsweise die Figur und der Hintergund unterschiedliche Farben besitzen, weil sie verschiedene Reflektanzprofile aufweisen. Und interessanterweise optiert Averill gerade für diese Theorie (vgl. Averill (1982): 24): für ihn haben zwei Gegenstände genau dann dieselbe Farbeigenschaften, wenn sie menschlichen Betrachtern unter allen möglichen Lichtbedingungen immer als gleichfarbig erscheinen (obwohl sie natürlich beide zusammen unter den einen Bedingun- 
gen als gelb und unter anderen als rot gesehen werden können). Dies ist genau dann der Fall, wenn sie dieselben Reflektanzprofile aufweisen (vgl. Hardin (1988): 73f.). Damit sind sie aber physikalische Arten. Es ist zudem richtig, daß die Individuation der Farben über alle möglichen Lichtbedingungen geht: Gegenstände derselben Farbe müssen immer metamerisch sein. In diesem Sinne ist er ein Starker Physikalist.

Nun interessiert Averill nur die Frage nach der Feingradigkeit der Individuation von Farben, nicht jedoch die konkrete Beschaffenheit und Wahrnehmung derselben. Würde er wirklich ganz auf Normalbedingungen verzichten, hieße das aber nichts anderes, als daß Gegenstände Farbeigenschaften relativ zu den Lichtbedingungen hätten, da unter verschiedener Beleuchtung ein- und dasselbe Reflektanzprofil unterschiedliche Farb wahrnehmungen bewirkt. Farbtatsachen wären damit ontologisch abhängig von den herrschenden Lichtbedingungen. Da ist die Alternative, nur die Farbwahrnehmungen, die unter Normalbedingungen auftreten, als veridisch anzusehen, wesentlich einsichtiger. Zudem widerspricht sie nicht der Ansicht Averills, Farbgleichheit nur bei strikter Isomerik (das heißt: Metamerik unter allen möglichen Beleuchtungen) anzunehmen und Farben über Reflektanzprofile zu individuieren. ${ }^{12} \mathrm{Da}$ er selbst übersieht, daß es sich bei seinem Vorschlag um eine Position handelt, die Farben als physikalische Arten ansieht (ob nun mit oder ohne Normalbedingungen), läßt sich wahrscheinlich nur durch Kripkes Vernachlässigen oder Übersehen der Disjunktivität von normalen Farbwahrnehmungsursachen erklären. Die von Kripke beschriebene Referenzfixierung muß der Starke Physikalist natürlich aufgeben.

Im Gegensatz zu Averill geht Tolliver von einem allgemeinen Problem für Objektivisten aus: und zwar die offensichtliche Phänomenalität der Farben $\mathrm{zu}$ erklären. Da ihn auch die üblichen, subjektivistischen Theorien nicht überzeugen, kommt er dazu, einen Informationalismus hinsichtlich von Farbwahrnehmungen (oder Farbempfindungen) anzunehmen, die subjektive Reaktionsdispositionen (response dispositions) der Gegenstände in

12 Averill bevorzugt die Redeweise, daß Farben über die Gleichheit oder Verschiedenheit der Beeinflussung von Licht unter allen Beleuchtungen individuiert werden (vgl. Averill (1982): 25). Aber genau dies leisten die Reflektanzprofile. Und zudem legt er viel Wert darauf, daß es sich bei seiner Theorie nicht um eine subjektrelative Farbanalyse handelt. 
der Welt detektieren. Dem Hauptproblem Averills, die Farbkonstanz nicht erklären zu können und, ohne Normalbedingungen, die Farbigkeit der Welt in gewissem Sinne von der gerade vorherrschenden Beleuchtung abhängig zu machen, entgeht Tolliver jedoch dadurch, daß er diese detektierten Dispositionen ganz einfach nicht als Farben betrachtet. Denn auf der sinnlich-phänomenalen Ebene der Wahrnehmungen gibt es für ihn noch gar keine Farben; erst durch die Anwendung von Farbbegriffen auf diese Sinneserfahrungen kommen Farbeigenschaften ins Spiel und können den wahrgenommenen Eigenschaften (fälschlicherweise) zugesprochen werden (vgl. Tolliver (1994): 433f.).

Ganz davon abgesehen, daß die (hier vielleicht nur terminologisch zu verstehende) Grundthese (IND) die Farben bereits auf der Ebene der Wahrnehmungen als präsentierte Eigenschaften ansieht, handelt es sich bei Tollivers Position im Grunde um eine Art von Subjektivismus: um einen Dispositionalismus auf der sinnlichen Ebene und um einen Projektivismus auf der begrifflichen Ebene. Der terminologische Punkt, ob etwas und, wenn ja, was dabei genau als Farben gelten sollte, ist nebensächlich. Die Probleme einer solchen Positionen ergeben sich aus der Annahme von detektierten Reaktionsdispositionen - und nicht so sehr aus der Annahme, daß es sich dabei um Farben handelt. Doch dieser Punkt soll erst im Subjektivismuskapitel ausgeführt und besprochen werden. Der Informationalismus stellt also nicht wirklich eine Alternative zu den anderen Theorien der Farben dar: entweder ist er gar keine Theorie über präsentierte Farben, oder er läßt sich auf eine andere Form der Theoretisierung reduzieren.

\subsection{Der Schwache Physikalismus}

Der Schwache Physikalismus sieht Farben nicht als physik-immanente, sondern als subjektrelativ charakterisierte Eigenschaften an. Damit ist die Farbigkeit (oder das Farbesein) an sich keine genuine physikalische Art oder Determinable, die sich auf fundamentalere physikalische Größen reduzieren ließe. Obwohl die einzelnen Farbeigenschaften $P_{\mathrm{i}}$ trotzdem physikalisch beschreibbar sind, weisen sie kein physik-relevantes, gemeinsames Merkmal $P$ auf, da ihre Natur wesentlich eine Subjektrelativität zeigt. Ihre physikalische Konstitution oder Realisierung kommt ihnen dabei nur kon- 
tingenterweise zu. Als Realisatoren fungieren letzten Endes intrinsische, physik-immanente Eigenschaften der farbigen Gegenstände: jede einzelne Instantiierung einer Farbe $P_{\mathrm{i}}$ entspricht somit eine Instantiierung einer physikalische Basis $B_{\mathrm{i}}$. Bei der Realisationsbeziehung zwischen Farben und ihren Basen handelt es sich dabei ganz allgemein um eine nichtreduktive Supervenienz (in dem Sinne, daß eine Reduktion der Farben auf die physikalische Basen wieder einen Starken Physikalismus mit sich brächte). Es ist zudem zu erwarten, daß multiple Realisation möglich ist, so daß für jede Farbe eine disjunktiv sich aus den einzelnen Basen zusammensetztende Basismenge existieren wird: ein Gegenstand kann die betreffende Farbe nur dann instantiieren, wenn er eine Basiseigenschaft aus der betreffenden Menge exemplifiziert.

Die Subjektrelativität impliziert, daß Farben nicht mehr als rein intrinsische Eigenschaften von Gegenständen aufgefaßt werden können, sondern als physikalistische Dispositionen: das heißt, Dispositionen, die über $\mathrm{Zu}-$ stände des visuellen Systemes von Subjekten oder über die Beschaffenheiten des von den Objekten ausgesandten Lichtes analysiert werden. Farben als Relationen oder nicht-physikalistische Dispositionen anzusehen, die beide auf mentale Zustände von Subjekten gerichtet sind, führt dagegen zu einem Subjektivismus. In diesem Abschnitt soll jedoch nur auf die objektivistische Variante des Dispositionalismus eingegangen werden, die bereits die Position des Schwachen Physikalismus vollständig erschöpft (wenigstens dem aktuellen Stand der Farbendebatte zufolge). Der Disjunktivismus stellt dabei eine Spielart dieses Dispositionalismus dar. Es sei angemerkt, daß der Begriff des Dispositionalismus in der Literatur meist restriktiver benutzt wird, als es hier der Fall ist: üblicherweise umfaßt er nur die subjektivistischen Positionen (vgl. zum Beispiel die Einleitung zu Byrne \& Hilbert (1997a): xxi, xxiii; oder Averill (1992): 551). Der hier verwendete Dispositionalismusbegriff ist weiter, da er jede Art von Dispositionalität der Farben umfaßt, also auch die objektivistischen Formen.

\subsubsection{Dispositionen}

Da Farben hier ausschließlich als Dispositionen, und nicht als physik-immanente Eigenschaften verstanden werden, lohnt es sich, die Ergebnisse ei- 
ner kategorialen Theorie der Dispositionen in Erinnerung zu rufen (eine ausführlichere Darstellung findet sich im Appendix A sowie in Armstrong (1997): 69ff.; 248ff.). Folgt man Armstrongs Vorschlag, so werden Dispositionen (in Absehung der relevanten Naturgesetzmäßigkeiten) letztlich ebenso feingradig individuiert wie ihre Basiseigenschaften. Das heißt aber nichts anderes, als daß Dispositionen sinnvollerweise mit den physikalischen Basen identifiziert werden und somit ihren dispositionalen Charakter verlieren: denn die einzelnen Basiseigenschaften besitzen jeweils kategorialen Status. Somit verschwinden Dispositionen vollkommen aus der realen Welt. Will man Farben also mit Dispositionen in diesem Sinne gleichsetzen, ergibt sich automatisch ein Starker Physikalismus. Denn die Basiseigenschaften von Dispositionen sind physik-immanente Eigenschaften: sie fungieren in kausalen Relationen und spielen in der Physik eine spezifische, ausgezeichnete Rolle. Diese Theorie der Dispositionen braucht uns hier also nicht mehr $\mathrm{zu}$ interessieren. Im Gegensatz dazu sehen Jackson, Pargetter und Prior (1982) Dispositionen als Eigenschaften zweiter Ordnung an: sie werden durch die physikalischen Basiseigenschaften realisiert.

Darüberhinaus wird davon ausgegangen, daß die These vom ontological free lunch Gültigkeit hat und Dispositionen wie auch Disjunktionen betrifft. Wäre dies nicht der Fall, müßten diese beiden Eigenschaftsarten als genuine Universalien aufgefaßt werden: neben den kategorialen gäbe es somit auch - ontologisch gleichberechtigt - dispositionale und disjunktive Universalien, die sich nicht auf die erste Art von Merkmalen zurückführen ließen. Gerade mit Bezug auf Dispositionen wäre diese Ansicht recht problematisch (vgl. Armstrong (1997): 83f.; 250ff). Die beiden wichtigsten Konsequenzen sind, erstens, die damit verbundene Existenz von notwendigen, dispositionalen Naturgesetzmäßigkeiten neben den kontigenten, kausalen und, zweitens, das unbestimmt bleibende Verhältnis zwischen ontologischen Bereichen des Kategorialen und des Dispositionalen, die doch offensichtlich in verschiedensten Wechselwirkungen zueinander treten (zum Beispiel vermittels der Realisieung und der Manifestierung von Dispositionen - vgl. Armstrong (1997): 83f.; 248ff.). Akzeptiert man also die These, daß dispositionale oder disjunktive Tatsachen keine genuinen Bestandteile zur Realität hinzufügen, die aus ontologischer Sicht über ihre Realisatortatsachen hinausgehen würden (oder in diesen nicht schon enthalten wären), 
dann wird der objektivistische Dispositionalismus gar nicht umhinkommen, Dispositionen mit den disjunktiven Basismengen gleichzusetzen. Damit ergibt sich eine wichtige Hypothese, die jedoch erst im weiteren Verlauf der Diskussion geklärt werden soll: Dispositionen sind nichts anderes als Disjunktionen (und umgekehrt).

In jedem Fall zeichnen sich Dispositionen als Eigenschaften zweiter Ordnung durch folgende Merkmale aus. Zuerst einmal werden Dispositionen über ihre möglichen Wirkungen - ihre Manifestationen - in der aktualen Welt individuiert: ein Gegenstand hat genau dann eine bestimmte Disposition, wenn er beim Auftreten ganz bestimmter, auslösender Umstände (und unter günstigen Hintergrundsbedingungen) aktual immer zu einer festgelegten Wirkung führt. Ein Stoff hat so zum Beispiel genau dann die Disposition, sich in Wasser zu lösen, wenn er sich auch faktisch in Wasser löst (Manifestation), sobald er mit ausreichend Wasser in Berührung gebracht wird (auslösender Umstand) und keine störenden Faktoren vorliegen (günstige Hintergrundsbedingungen). Zudem supervenieren Dispositionen, wie gesagt, auf bestimmten physikalischen Basiseigenschaften: Tatsachen über die Basen fungieren somit als Wahrmacher für dispositionale $\mathrm{Zu}$ schreibungen. Hinsichtlich einer bestimmten Disposition gelten genau die Eigenschaften als Basen - und somit als Disjunkte der entsprechenden, disjunktiven Basismenge -, die unter den beschriebenen Bedingungen aktual zu der die Disposition definierenden Wirkung führen.

Die Einschränkung auf die aktuale Welt ist durch den Umstand bedingt, daß die Manifestierung einer Disposition auch davon abhängt, ob die relevanten Naturgesetzmäßigkeiten, die die Basiseigenschaften und die auslösenden Umstände mit der Manifestation verbinden, Bestand haben. Es sind zum Beispiel Welten denkbar, in denen Salz sich nicht auflöst, wenn es in einen Behälter mit ganz normalem Wasser geschüttet wird: in der also eine Basiseigenschaft und die relevanten, auslösenden Umstände vorliegen, aber sich keine Manifestierung ereignet. Da Salz jedoch in der aktualen Welt diese Löslichkeit zeigt, sprechen wir ihm sinnvollerweise die Disposition zu, sich in Wasser lösen zu können. Damit wird auch die für den Farb objektivisten so wichtige Rigidität gewährleistet: ob ein Gegenstand eine bestimmte Disposition hat, hängt allein davon ab, ob er sie in der aktualen Welt instantiiert. In Welten, wo die für die Manifestierung erforderliche Naturgesetzmäßigkeit nicht besteht, ist dann einfach die Möglichkeit der 
Manifestierung nicht mehr gegeben. Aber es ist ontologisch kein Problem, von unmanifestiert bleibenden Dispositionen auszugehen. Daneben ist einund dieselbe Disposition multipel realisierbar; das heißt, eine Disposition kann, wenn sie von unterschiedlichen Gegenständen instantiiert wird, jeweils durch verschiedene Basen realisisiert sein. Die einzelnen, möglichen Basiseigenschaften einer Disposition können, wie bereits erwähnt, in der disjunktiven Basismenge zusammengefaßt werden (disjunktiv, da eine Instanz der Disposition immer nur genau eine dieser Basen exemplifiziert). Diese disjunktive Menge

Im Falle der Manifestierung einer Disposition liegt immer eine singuläre Kausalrelation vor, welche von der betreffenden Basis und den auslösenden Umständen ceteris paribus zur Manifestation führt. Die Disposition selbst ist dabei epiphänomenal, da die Basis und die auslösenden Ursache zusammen mit den bestehenden Hintergrundsbedingungen bereits hinreichend sind, die Manifestierung zu bewirken. Zwar können dispositionale Zuschreibungen informativ sein (wie etwa die Aussage: „Salz ist in Wasser löslich“); doch für dispositionalen Gesetze - die die Disposition über die auslösenden Umstände mit der Manifestation in einen nomologischen $\mathrm{Zu}$ sammenhang setzen - besteht diese Möglichkeit nicht, da sie einen tautologischen Charakter haben. Daß Salz in Wasser löslich ist, kann nicht durch das dispositionale Gesetz erklärt werden, daß Salz, wenn es in Wasser geschüttet wird, sich auflöst. Dispositionalen Gesetze sind für sich genommen immer uninformativ. Erst durch die Einbeziehung der kausalen Ebene - das heißt: der kausal wirksamen Basiseigenschaften und der relevanten, kausalen Gesetzmäßigkeiten - können epistemisch wertvolle Erklärungen für das Auftreten von Manifestationen gegeben werden. Die allgemein als Disjunktionen bestimmten Dispositionen bleiben selbstverständlich epiphänomenal. $\mathrm{Ob}$ sie trotzdem eine Erklärungskraft haben können, wird sich erst bei der konkreten Anwendung auf die Farbproblematik zeigen.

Der Schwache Physikalist setzt nun Farben mit bestimmten, physikalisch beschreibbaren Dispositionen der als farbig erscheinenden Gegenstände gleich. Die Konkretierung des physikalistischen Dispositionalismus erfordert jedoch die Konkretisierung der Eigenschaften in der Welt, die als Farbdispositionen in Frage kommen. Wieder kann die naturwissenschaftliche Erforschung von Farbphänomenen hier weiterhelfen. Dabei wird es sich zeigen, daß der Schwache Physikalismus gegenüber dem Starken den 
Vorteil hat, daß sich für ihn das oben beschriebende Problem der Repräsentierbarkeit nicht stellt. Vielmehr kann, aller Voraussicht nach, sogar die Erklärungskraft der Farbdispositionen aufrechterhalten werden.

\subsubsection{Farben als Reflektanztypen}

Die Grundidee ist es nun, die Metamerik der Reflektanzprofile auszunützen, indem die jeweils unter Normalbedingungen metamerischen, spektralen Oberflächenreflektanzen in Klassen zusammengefaßt werden. Die eine bestimmte Klasse bildenden Reflektanzprofile zeichnen sich dadurch aus, daß sie von ein- und demselben Reflektanztypus sind. Somit werden die durch die Metamerik und die Unbestimmtheit der Wahrnehmung hervorgerufenen Probleme für den Starken Physikalismus umgangen (und zwar auf Kosten der Physik-Immanenz). Nun lassen sich Entitäten im allgemeinen über beliebig viele verschiedene Kriteria in ganz unterschiedliche Klassen unterteilen. Es wird also Ziel der Colorimetrie sein, die farbrelevanten Typen von Reflektanzen zu identifizieren. Vor dem Hintergrund der These $\left(\mathrm{IND}_{\mathrm{R}}\right) \mathrm{mu}$ es sich für den Farbphysikalisten dabei um einen von unseren Farbwahrnehmungen repräsentierten Reflektanztypus - oder eine repräsentierte Klasse von Reflektanzprofilen - handeln. Mittels der These $\left(\mathrm{ACT}_{\mathrm{NB}}\right)$, welcher sich auch die Colorimetrie bedient, läßt sich somit schließen, daß farbrelevante Reflektanztypen diejenigen sind, die mit unseren Farbwahrnehmungen unter Normalbedingungen nomologisch korreliert sind. ${ }^{13}$ Dabei werden die Sinneserfahrungen über den phänomenalen Aspekt ihres introspektiven Charakters individuiert. Die zu suchenden Klassen von Reflektanzprofilen müssen also jeweils mit den entsprechenden Farbqualia korreliert sein.

In dem Kapitel über die Wissenschaft der Farben sind die betreffenden Reflektanztypen $S S R_{\mathrm{T}}$ bereits kurz angesprochen worden. Die Colorimetrie

13 Damit verbunden ist die praktische Schwierigkeit, daß die Normalbedingungen nur zusammen mit der repräsentierten Eigenschaft gefunden werden können: zwar ist vor Beginn der empirischen Forschung ganz grob klar, was als normal und was als unnormal zu gelten hat; aber eine Konkretisierung der Normalbedingungen kann erst im nachhinein, mit Bezug auf die Eigenschaften, vollzogen werden. 
hat für sie eine nomologische Korrelation unter Normalbedingungen mit Farbqualia nachgewiesen. Sie sind also für einen Farbphysikalisten die naheliegensten Kandidaten für die Identifizierung der Farben mit physikalisch beschreibbaren Eigenschaften $P$. Während der Starke Physikalismus diese Gleichsetzung zu vermeiden sucht, da sie, wie sich zeigen wird, keine Physik-Immanenz der Farbeigenschaften zuläßt, nimmt der Schwache Physikalismus diese Konsequenz in Kauf, um dem Problem mit der Repräsentierbarkeit von Farben entgehen zu können. Dementsprechend identifiziert letzterer Farben mit den von Farbwahrnehmungen repräsentierten Reflektanztypen $S S R_{\mathrm{T}}$. Die durch diese grundsätzliche Idee charakterisierte Position findet sich bereits in Hilberts Buch Color and Color Perception (1987) und ist dann in seinem zusammen mit Byrne geschriebenen Aufsatz Colors and Reflectances (1997c) erweitert und ausgearbeitet worden. Der Schwache Farbphysikalismus läßt sich somit durch die folgende These charakteristieren: ${ }^{14}$

(PHY $\left._{\text {SCHWACH }}\right) \quad P:=$ Reflektanztypen $S S R_{\mathrm{T}}$.

Bringt man sich die naturwissenschaftlich gewonnenen Erkenntnisse über die Farbigkeit von externen Gegenständen in Erinnerung, so mag die Frage aufkommen, warum der Starke Physikalismus nicht auch Reflektanztypen als mögliche Kandidaten für die Identifikation mit Farben betrachten kann. Die Antwort ist sehr einfach: die relevanten Reflektanztypen sind keine physik-immanenten Eigenschaften, das heißt, sie bilden für sich genommen keine physikalische Art oder Größe. Zuerst muß jedoch noch gezeigt werden, daß es sich bei diesen Reflektanztypen - oder den entsprechenden

14 Vgl. Byrne \& Hilbert (1997c): 265f. - Der gleichen Auffassung über Farben ist aller Voraussicht nach auch Broackes, der Westphals Vorschlag, Farben als „Weisen der Lichtbeeinflussung" anzusehen (ways of changing light) so interpretiert, daß die resultierende Grundansicht der von Byrne und Hilbert sehr nahe kommt (Broackes (1992): 454ff.; 459). Auch wenn die Disjunktivisten diese Identifikation mit Reflektanztypen nicht explizit vornehmen, können auch sie als Vertreter des durch ( PHY $\left._{\text {SCHWACH}}\right)$ spezifizierten Schwachen Physikalismus gelten. Eine Farbdisjunktivismus vertreten: Smart (1975); Armstrong (vgl. (1987); (1993); (1997)); und Shoemaker (vgl. (1986); (1994b)). Lewis wird meistens ebenfalls noch dazugezählt (vgl. Armstrong (1987): 15). Armstrongs Position bedarf später jedoch noch einer genaueren Untersuchung. 
Klassen von Reflektanzprofilen - wirklich um physikalistisch beschreibbare Entitäten handelt. Reflektanztypen $S S R_{\mathrm{T}}$ werden im allgemeinen entweder als Tripel von jeweils über den drei Wellenlängenbereichen $L, M$ und $S$ (für die die Rezeptoren unserer Augen sensitiv sind) integrierten Oberflächenreflektanzen definiert oder aber mithilfe der im visuellen System realisierten Kodierung der Gegenfarbtheorie spezifiziert. Dabei können beide Spezifizierungen hier als in etwa gleichwertig betrachtet werden; doch im weiteren Verlauf der Diskussion soll letztere bevorzugt verwendet werden, da sie den Vorteil bietet, Farben über Zustände des visuellen Systemes zu charakterisieren.

Der erste Weg versteht Reflektanztypen nun also als Tripel aus Proportionswerten, die jeweils für einen der drei Wellenlängenabschnitte angeben, wieviel einfallendes Licht in diesem Bereich von dem betreffenden Gegenstand insgesamt reflektiert wird. Diese Proportionswerte werden dabei durch Integration (entsprechend der Sensitivitätskurve der fraglichen Rezeptorenart) über die einzelnen, wellenlängen-spezifischen Reflektanzen der Oberfläche gewonnen. Er führt zu Definitionen wie der folgenden, die dem reinen Rot ein ganz bestimmtes Tripel über $L, M$ und $S$ integrierter Reflektanzen zuordnet: nämlich 34,15 und $11 \%$ (vgl. McCann, McKee \& Taylor (1976): 456). Für dieselbe Farbe ergibt sich nach der zweiten Alternative die folgende Charakterisierung: reines Rot ist die Eigenschaft, unter normaler Beleuchtung im langwelligen Bereich $L$ mehr Licht zu reflektieren als im mittelwelligen $M$, und zudem im kurzwelligen $S$ in etwa genau soviel wie in den anderen beiden Bereichen zusammen (oder in Formeln: $L$ $>M ; S » L+M$ ). Diese Kodierungen der Farbe mithilfe der drei durch Integration gewonnenen Intensitätswerte für die drei Wellenlängenbereicht $L$, $M$ und $S$ spielen eine wesentliche Rolle in der sogeannten Gegenfarbtheorie, die die Zuordnung dieser durch das visuelle System aufgenommenen Informationen über die Intensitäten des einfallenden Lichtes zu den resultierenden Farbempfindungen beschreibt. Beide alternativen Bestimmungen der Reflektanztypen weisen nun eindeutig darauf hin, daß es sich bei diesen Eigenschaftsarten um physikalische (im weiten Sinne) handelt.

Aber Reflektanztypen sind kein physik-immanenten Eigenschaften: das heißt, sie stellen keine physikalische Art dar (vgl. Hilbert (1987): 100; und Tolliver (1994): 417). Zwar kann die Klasse der Reflektanztypen-an-sich ganz allgemein als integrierte Reflektanzen über einen oder mehrere, wie 
auch immer eingegrenzte Wellenlängenbereiche verstanden - vielleicht noch als eine physikalische Art oder Größe definiert werden; aber die Einschränkung auf Reflektanztripel hinsichtlich fixierter Wellenlängen $a b$ schnitte bedient sich Faktoren, die nicht physik-immanent sind, so daß dies ebenfalls für die resultierende Teilmenge aus der Klasse aller überhaupt möglichen Reflektanztypen-an-sich gilt. Die für das menschliche Farbensehen bedeutsamen Reflektanztypen sind beispielsweise allein auf die drei konkreten Wellenlängenbereiche $L, M$ und $S$ des von Menschen wahrnehmbaren Spektrums beschränkt (und für andere Lebewesen ergeben sich ganz ähnliche Einschränkungen). Somit ist nur eine ganz bestimmte Teilmenge der Gesamtheit der Reflektanztypen-an-sich für die Farberkennung wesentlich, während die physikalische Art Reflektanztypus an sich hierfür keine ausgezeichnete Bedeutung hat. ${ }^{15}$ Da Reflektanztypen letztlich nur subjektrelativ individuiert werden können, fällt ihre mögliche Identifizierung mit Farben in den Bereich des Schwachen Physikalismus. Auch Byrne und Hilbert, die die bekanntesten Verfechter dieser Identifikation sind, kommen zu diesem Schluß:

„The property green, if it is [a] type of SSR, is not a particularly interesting property from a physical point of view. Since we only find it salient because our perceptual apparatus is built to detect it, it might be called an anthropocentric property.“ (Byrne \& Hilbert (1997c): 266) ${ }^{16}$

Im Grunde läßt sich die Identifikation von Farben mit Reflektanztypen ontologisch auf zwei Weisen interpretieren (vgl. Hilbert (1987): 110f.). Einerseits kann die Lesart als Klasse von Reflektanzprofilen bevorzugt werden;

15 Daß keine Physik-Immanenz vorliegt, ist vor allem auf den Umstand zurückzuführen, daß es sich um Reflektanztripel handelt, wie es durch die Anzahl der Rezeptoren im menschlichen Auge vorgegeben wird. Solch eine auf die Konstitution von Subjekten bezogene Beschränkung kann es innerhalb der Physik nicht geben. Reflektanzprofile sind dagegen physik-immanent, da sie einfach über physikalische Größen und Gesetzmäßigkeiten bestimmt werden. Die Existenz von subjektrelativen, wahrnehmbaren Spektren spielt hingegen bei beiden keine Rolle für den ontologischen Status (vgl. Fn. 8 oben).

16 Eine ausführliche Vorstellung und Diskussion dieses sogenannten Anthropozentrischen Farbrealismus findet sich bei Hilbert, der diese Position schon früher vertreten hat (vgl. Hilbert (1987): zum Beispiel 15). 
Farben sind damit nichts anderes als Disjunktionen von physikalischen Eigenschaften. Andererseits kann man jedoch auch versuchen, Reflektanztypen als Dispositionen anzusehen. In beiden Fällen handelt es sich also um Eigenschaften zweiter Ordnung. Das Ziel des nächsten Abschnittes wird es sein, zu zeigen, daß die disjunktivistische und die dispositionalistische Analyse von Farben im Grunde ein- und dieselbe Position darstellen. Damit soll die durch die allgemeine, kategoriale Theorie der Dispositionen vorgestellte Hypothese auf den konkreten Fall der Farben angewandt werden: Farbdisjunktionen sind Farbdispositionen (und umgekehrt). Diese These soll nun im folgenden noch einmal plausibel gemacht werden. Aus systematischen Gründen ist es angebrachter, diesmal die Disjunktionen auf die Dispositionen zurückzuführen. Ontologisch betrachtet sind beide Redeweisen jedoch äquivalent: insbesondere weisen beide Konzeptionen Schwierigkeiten hinsichtlich ihrer Einbeziehung in eine kategoriale, nichtdisjunktive Realität auf. Beginnen wir also mit der Besprechung der disjunktivistischen Position.

\subsubsection{Farben als Disjunktionen}

In der Aufsatzsammlung zu Ehren Smarts (vgl. Pettit, Sylvan \& Norman (1987)) wird sowohl von Armstrong als auch von Smart selbst des letzteren Ansatz für eine Theorie der Farben in ihrer Entwicklung verfolgt, bis sie schließlich die Form eines Disjunktivismus oder Disjunktiven Realismus angenommen hat. In verschiedenen Varianten ist diese Position bis heute für viele Philosophen attraktiv geblieben, doch die klassische, physikalistische Auffassung ist immer noch die von Smart, wie er sie in einem 1968 in Hawaii gehaltenen und erst sieben Jahre später veröffentlichten Vortrag dargestellt hat (vgl. Smart (1975)). Es ist, wie bereits erwähnt, eine Tatsache, daß faktisch ganz verschiedene physikalische Eigenschaften von Gegenständen für Farbwahrnehmungen in menschlichen Betrachtern verantwortlich sein können. Bei diesen Eigenschaften handelt es sich um die Reflektanzprofile, die je nach der von ihnen aktual unter Normalbedingungen hervorgerufenen Farbempfindungstypen in einzelne Klassen zusammengefaßt werden können. ${ }^{17}$ Diese Klassen sind deshalb disjunktiv zu nen-

17 Erst mit den Texten von Hilbert scheint die Auffassung (wieder) prominent ge- 
nen, weil ein Gegenstand, der die entsprechende Farbempfindung bewirkt, genau eine Eigenschaft aus dieser Menge instantiieren kann und muß.

Wie bei Dispositionen bestehen nun die beiden Möglichkeiten, Farben mit den einzelnen Reflektanzprofilen oder aber mit der zu einem Farb wahrnehmungstypus gehörenden Disjunktion von Reflektanzprofilen zu identifizieren. Die erste Alternative (welche weder von Smart, noch - vermutlich - von Armstrong in Betracht gezogen wird: vgl. Smart (1975): 57; sowie Armstrong (1987): 8f) führt offensichtlich zu dem bereits beschriebenen Starken Physikalismus, weswegen wir sie hier ignorieren können. Bleibt also die Variante, Farben tatsächlich mit den Disjunktionen gleichzusetzen. Dann kann von den einzelnen Reflektanzprofilen als Realisierungsbasen (oder Disjunkten) der betreffenden Disjunktion (oder Basismenge) gesprochen werden. Instantiierungen einer solchen Farbdisjunktion supervenieren auf der Instantiierung einer entsprechenden Basiseigenschaft. Diese Disjunktionen gelten nun als Farben aus dem einfachen Grund, da sie faktisch von Farbwahrnehmungen repräsentiert werden, das heißt, unter Normalbedingungen mit Farbwahrnehmungstypen nomologisch korreliert sind: jedes Disjunkt einer gegebenen Disjunktion verursacht (für sich allein genommen) normalerweise ein- und denselben, über den phänomenalen Charakter individuierten Farbwahrnehmungstypus (vgl. dazu auch. Armstrong (1987): Fn. 13). Damit sind sowohl (IND $\left.{ }_{\mathrm{R}}\right)$ als auch $\left(\mathrm{ACT}_{\mathrm{NB}}\right)$ erfüllt.

„Colours are the (perhaps highly disjunctive and idiosyncratic) properties of the surfaces of objects that explain the discrimination with respect to colour of normal human percipients, and [that explain - der Autor] also the experiences of these percipients, the looking red, or looking blue, etc. of objects." (Smart (1975): 60)

worden zu sein, diese Eigenschaften nicht auf der mikro-, sondern der makrophysikalischen Ebene zu suchen: auch wenn bereits in dem frühen Text von Keith Campbell Reflektanzeigenschaften explizit in einem Atemzug mit dem Disjunktivismus genannt werden (vgl. K. Campbell (1969): 137). Smart, Armstrong und die meisten anderen sprechen dagegen nur von mikrophysikalischen Eigenschaften, ohne diese weiter zu benennen. Im Lichte der obigen Erwägungen genügt es auch hier, sich auf die makroskopische Ebene zu beschränken, da sie naturwissenschaftlich angemessen die mikroskopische mit abdeckt. Für die Argumentation selbst spielt diese Entscheidung keine Rolle. 
Dabei ist die Faktizität dieser Aussage besonders hervorzuheben: Smart akzeptiert die Möglichkeit, daß dieselben physikalischen Beschaffenheiten von Gegenständen unter anderen Bedingungen (wie Qualia-Inversion oder -Abwesenheit) entweder zu phänomenal anderen Farbwahrnehmungen, oder auch zu gar keinen mehr, führen können (vgl. Smart (1975): 59; vgl. dazu Armstrong (1987): 6f.; 11). Diese Einschränkung auf die aktuale Welt findet sich auch bei anderen Autoren, die einem Disjunktivismus nahestehen (insbesondere Armstrong, der Smarts Analyse weitestgehend akzeptiert), oder aber diese Position darstellen (vgl. etwa K. Campbell (1993): 258f.). In der aktualen Welt wird eine ganz bestimmte Disjunktion mit der Farbe Rot gleichgesetzt werden; in einer anderen kann es aufgrund von Änderungen der Naturgesetzmäßigkeiten jedoch ohne weiteres der Fall sein, daß einzelne dieser Disjunkte, oder auch all, nicht mehr zu Rotwahrnemungen führen, sondern vielleicht zu Blauwahrnehmungen, oder zu gar keinen Farbwahrnehmungen mehr. ${ }^{18}$

Welche Disjunkte dabei zu der mit einer bestimmten Farbe identifizierten Disjunktion gehören, wird immer nur über deren kausale Wirksamkeit in der aktuale Welt festgelegt. Damit ist Rigidzität garantiert: ein roter Gegenstand, dessen intrinsische Beschaffenheit sich nicht verändert, behält seine Farbe in allen möglichen Welten bei, auch wenn er längst nicht in allen normalerweise zu Rotwahrnehmungen führen wird. Allein die aktuale Welt ist für die Farbklassifikationen ausschlaggebend: den einzelnen Farben werden bestimmte Disjunktionen von Reflektanzprofilen zugeordnet, weil menschliche Betrachter in der aktualen Welt die Instanzen der Disjunkte der letzteren unter Normalbedingungen als entsprechend gefärbt sehen. Deswegen sind Farben laut dieser Theorie nicht nur disjunktiv, sondern auch idiosynkratisch: doch beide Merkmale schienen Smart nicht unproblematisch.

18 Tolliver verbindet hiermit auch das Problem, daß die Disjunktionen unbestimmbare, offene Mengen bleiben müssen, da die Disjunkte über eine Farbdispositionalität spezifiziert werden und es beliebig viele Basen für die entsprechenden Dispositionen geben kann: die Disjunktion kann somit nicht zur Analyse der Farben verwendet werden (vgl. Tolliver (1994): 416). Tolliver übersieht jedoch, daß genau diese Dispositionalität als wesentliches Merkmal der Farben genommen werden kann, ohne den Objektivismus aufzugeben. Denn im Grunde sind Disjunktionen nichts anderes als Dispositionen. 
„Colours are physical properties of the surfaces of objects. [...] My worry was that these properties must be highly disjunctive and idionsyncratic ones. Lewis said 'Disjunctive and idiosyncratic, so what?' Though disjunctive and idiosyncratic (that is, of interest to humans but not, say, to Alpha Centaurians) they could still be perfectly physical properties. To adapt an analogy used by Robert Boyle, the shape of a lock might be of interest only because of a certain shaped key, but the shape of the lock is something perfectly physical - indeed geometrical which can be described without reference to the key." (Smart (1987): 175)

Mit den möglichen Schwierigkeiten für die Anthropozentrik oder Idiosynkrasie einer Theorie der Farben werden wir uns im nächsten Abschnitt, im Zusammenhang mit dem Dispositionalismus, beschäftigen. Daß den Physikalisten die Disjunktivität zuerst nicht behagt hat, läßt sich auf drei Argumentationen zurückführen. Die folgende, klassische findet sich in einem frühen Text von Keith Campbell, in dem er das sogenannte „Axiom der Einheit" (axiom of unity) vertritt, demzufolge ein Farbphysikalismus zu jeder einzelnen Farbe angeben können muß, was ihr spezifisches, physik-immanentes Kriterium ist. Das heißt aber nichts anderes, daß Campbell dort noch gefordert hat, Farben müßten irgendwie beschaffene, physikalische Arten sein, damit ein Farbphysikalismus wahr sein könnte (vgl. K. Campbell (1969): 133; 137f.). Vor dieser Hintergrundannahme läßt sich der Disjunktivismus leicht als ein physikalistische Position zurückweisen. Doch wie bereits argumentiert worden ist, besteht bereits dann ein Physikalismus, wenn (ganz grob) Farben als physikalisch beschreibbare Eigenschaften externer Gegenstände angesehen werden können: sie müssen dagegen nicht physik-immanent sein. Das „Axiom der Einheit“ gilt zwar für einen Starken Farbphysikalismus, nicht jedoch für einen Schwachen. ${ }^{19}$ Damit ist Campbells Argument hinfällig, wie er selbst dadurch zugibt, daß er die Disjunktivität als solche später nicht mehr als problematisch empfindet

19 Interessanterweise vertritt gerade Campbell in diesem frühen Aufsatz einen schwach-physikalistischen Disjunktivismus mit Farben als offene und disjunktive Klassen von physikalischen Eigenschaften, die faktisch kausal wirksam sind hinsichtlich von Farbempfindungen (vgl. K. Campbell (1969): 157). Später dann argumentiert er gegen den Disjunktivismus, ohne jedoch eine alternative Position vorzuziehen: im Grunde kommt der Disjunktivismus trotz seiner Kritik bei ihm noch am besten weg (vgl. Campbell (1993)). 
(vgl. K. Campbell (1993): 258f.).

Ein zweites Problem für den Disjunktivisten ist es, daß er die introspektiv erkannte Einheit von phänomenalen oder präsentierten Farben als eine Art von systematischem Fehler in unserer Farberkennung ansehen muß. Da es sich hierbei jedoch um ein Merkmal des phänomenalen, und nicht des repräsentationalen, Aspektes des introspektiven Charakters handelt, ist dieser Einwand hier direkt noch von keiner besonderen Bedeutsamkeit: er wird im Zusammenhang mit der generellen Kritik, daß ein Farbphysikalismus der Phänomenalität von Farben nicht gerecht werden kann, wieder aufgenommen (vgl. den Abschnitt gegen den Objektivismus ${ }_{\mathrm{ACT}}$ ). Ein dritter, ehemals von Armstrong vorgebrachter Einwand zweifelt die Existenz von disjunktiven Eigenschaften an. ${ }^{20}$ Nimmt man disjunktive Universalien an, dann kann es der Fall sein, daß für ihre Instanzen nur die sogenannte Cambridge-Ähnlichkeit gilt (aber keine genuine): wenn ein Gegenstand das Universale $A$ und ein anderer das Universale $B$ instantiiert, dann sind sie sich darin ähnlich, daß sie beide die disjunktive Eigenschaft $A \vee B$ exemplifizieren. Falls die Disjunktion ein genuines Universale sein könnte, dann könnten in diesem Sinne beliebige Gegenstände sich ähnlich sein, wenn nur das geeignete disjunktive Universale konstruiert werden könnte. Solch ein Umstand ist vielleicht möglich, aber sehr plausibel ist er nicht. Doch Armstrong ist zu der Einsicht gelangt, daß man trotzdem, im Gegensatz zu seiner früheren Meinung, disjunktive Eigenschaften einem Gegenstand korrekterweise zuschreiben kann. Und zwar werden Disjunktionen nun von ihm nicht mehr als Universalien, sondern als Eigenschaften zweiter Klasse aufgefaßt, für die der ontological free lunch gilt, da sie auf ihren Disjunkten supervenieren. Somit ist auch dieser mögliche Einwand hinfällig.

Wie kommt man aber von Disjunktionen zu Dispositionen? Beide sind Eigenschaften zweiter Ordnung, doch anscheinend weisen sie ganz verschiedene, ontologische Beschaffenheiten auf. Doch beide supervenieren

20 Dies ist auch der Grund, warum Armstrong ursprünglich die Nichtexistenz von Farben als eine mögliche Alternative gesehen hat: wenn Farben unausweichlich disjunktiv sind und es keine disjunktiven Universalien geben kann, dann gibt es eben auch keine Farben (vgl. Armstrong (1987): 9). Deswegen hat er auch eine Vereinheitlichung der physikalischen Natur der Farben durch einen Starken Physikalismus gefordert, um dem Eliminativismus zu entgehen. Später dann hat Armstrong seine grundsätzliche Haltung zu diesem Punkt geändert. 
auf denselben kategorialen und nicht-disjunktiven Eigenschaften. So bilden Disjunktionen einen besonderen Komplex von Eigenschaften, welcher in seiner Struktur der mit Dispositionen verbundenen Basismengen gleicht. Und gerade über diese Ähnlichkeit läßt sich ein Zusammenhang zwischen Disjunktionen und Dispositionen herstellen. Welche Eigenschaften zu der Basismenge einer mit einer bestimmten Farbe identifizierten Disposition gehören, hängt von demselben Kriterium ab, wie der Umstand, welche Eigenschaften als Disjunkte einer mit derselben Farbe gleichgesetzten Disjunktion zugerechnet werden: in beiden Fällen entscheidet die aktuale, kausale Wirksamkeit der betrachteten physikalischen Eigenschaften hinsichtlich der entsprechenden Farbempfindung. Die Disjunktion wird erst dadurch zu einer Farbe, daß alle ihre Disjunkte (und nur diese) die Eigenschaft zweiter Ordnung zeigen, unter Normalbedingungen faktisch zu einer ganz bestimmten Farbempfindung $\mathrm{zu}$ führen. Und dieselbe Eigenschaft zweiter Ordnung gruppiert dieselben Basiseigenschaften in die korrespondierende Basismenge der Disposition.

Bei dieser Eigenschaft zweiter Ordnung handelt es sich natürlich um die betreffende Disposition. Die Differenz zwischen dem Dispositionalismus und dem Disjunktivismus besteht also darin, daß erstere Farben mit Dispositionen, letzterer dagegen mit den dazugehörigen disjunkten Basismengen identifizieren. Disjunktionen können überhaupt erst als Farben gelten, wenn sie mit Farbdispositionen in Verbindung gebracht werden. Dabei unterscheiden sich beide Positionen ontologisch gleichermaßen von dem Starken Physikalismus, der Farben mit den Basiseigenschaften, also den einzelnen Disjunkten, gleichsetzt. Und beide Positionen sind, ontologisch betrachtet, äquivalent. Diese These ist mit zwei besonders wichtigen Punkten verträglich. Erstens wird Farben in beiden Fällen wesentlich eine dispositionale Natur zugesprochen: welche Gegenstände aufgrund welcher physik-immanenten Eigenschaft welche Farbe instantiieren, hängt allein davon ab, wie sie Betrachtern unter Normalbedingungen erscheinen. Zweitens teilen beide das Merkmal, keine Eigenschaften im eigentlichen Sinne zu sein, da sie nicht auf genuine Universalien reduziert werden können, sondern Eigenschaften zweiter Ordnung darstellen.

Für Dispositionen ist dieser Punkt bereits eingehend erläutert worden. Mit Bezug auf Disjunktionen ist noch einiges zu sagen. Es ist offensichtlich, daß es sich bei letzteren ebenfalls nicht um Eigenschaften im eigentli- 
chen Sinne handelt, da es keine disjunktiven Universalien als realer Bestandteil der Welt gegen kann. Akzeptiert man jedoch den ontological free lunch, dann lassen sich Disjunktionen - wie Dispositionen - als supervenierende Eigenschaften zweiter Ordnung konstruieren. Nun werden Disjunktionen sicherlich immer von genau einem ihrer Disjunkte realisiert: ein Gegenstand exemplifiziert sozusagen genau dann eine Disjunktion, wenn er eines der Disjunkte instantiiert. Doch es läßt sich noch mehr sagen. Aufgrund der Kontingenz von Naturgesetzmäßigkeiten kann es ebenso wie bei den Dispositionen vorkommen, daß die in der aktualen Welt unter normalen Umständen zu einer Rotwahrnehmungen führendene Eigenschaft in anderen Welten diese Wrkung nicht mehr zeigt. Zudem sind auch Disjunktionen multipel realisierbar durch jedes einzelne ihrer Disjunkte; in beiden Fällen verursachen die zugrundeliegenden Basiseigenschaften unter den entsprechenden Bedingungen Farbwahrnehmungen. Und sowohl Dispositionen als auch Disjunktionen sind epiphänomenal (jeweils nur eines der Disjunkte ist kausal wirksam) und erhalten ihre Erklärungskraft allein durch Einbeziehung der kausalen Ebene. ${ }^{21}$

Dispositionalismus und Disjunktivismus sind somit eigentlich nur zwei verschiedene Beschreibungen ein- und desselben Sachverhaltes. Dies ist auch bereits in der Diskussion der Natur von Dispositionen klar geworden, in der die beste, verfügbare Theorie Dispositionen als Disjunktionen der Basiseigenschaften angesehen hat. Deswegen kann letztere Position durch eine ontologisch irrelevante Umdeutung in erstere überführt werden: Disjunktionen als Farben verstanden sind nichts anderes als Dispositionen als Farben verstanden. Der Dispositionalismus wird hier bevorzugt, da er die von beiden Theorien bestimmte, wesentliche Charakteristik der Farben angemessener beschreibt. Der Disjunktivismus ist sicherlich von den meisten überzeugten Physikalisten bevorzugt worden, da er den objektiven Charakter der Farben stärker herauszuarbeiten oder zu betonen scheint und zudem den ontologisch problematischeren Begriff der Dispositionalität vermeiden hilft. Dies ist aber nur korrekt, wenn man die dispositionalistische Position (wie oft geschehen) darauf beschränkt, daß Farben als Disposition eine sehr enge Beziehung zu mentalen Zuständen aufweisen. Und schließlich

21 Laut Armstrong ist dies der Grund, warum Lewis von Smarts Analyse der Farben, welche ja auf eine seiner Ideen zurückzuführen ist, letztlich wieder abgerückt ist (vgl. Armstrong (1987): 10). 
scheint der dispositionale Charakter unserem Eindruck von den in Farb wahrnehmungen präsentierten Eigenschaften näher zu kommen als ein disjunktiver (auch wenn es sich nur um verschiedene Beschreibungen einund derselben ontologischen Eigenschaftsart handelt). In jedem Fall tritt die Subjektrelativität oder, nur auf den Menschen bezogen, die Anthropozentrik (oder auch Idiosynkrasie) in den Vordergrund. Es genügt also, sich weitestgehend auf den Dispositionalismus zu konzentrieren.

\subsubsection{Farben als subjektrelative Dispositionen}

Um repräsentierte Farben allgemein als Dispositionen ansehen zu können, sollte kurz noch einmal auf die dispositionale und damit subjektrelative Natur der Reflektanztypen $S S R_{\mathrm{T}}$ hingewiesen werden. Wie bei der alternativen Interpretation als Disjunktionen ist das ihnen als Farben gemeinsam zukommende Merkmal die Disposition, unter normalen Umständen faktisch bestimmte, über ihren phänomenalen Aspekt individuierte Farbwahrnehmungen in einem Betrachter hervorzurufen. Diese Dispositionalität hängt zum einen von den physik-immanenten Basiseigenschaften - den Reflektanzprofilen - und zum anderen von den bestehen Gesetzmäßigkeiten $a b$, die die Basen mit der Wirkung - also dem hervorgerufenen Farbquale - kausal verbinden. Die Reflektanztypen zeigen ebenso die üblichen Merkmale einer Disposition: sie sind Eigenschaften zweiter Ordnung, multipel realisierbar, epiphänomenal und nur dann erklärend, wenn die mögliche kausale Realisierung ihrer Manifestierung in Betracht gezogen wird (vgl. Hilbert (1987): 25ff.; 119ff.). Zudem sind sie - mehr oder weniger subjektrelativ oder anthropozentrisch.

„The identification of color with surface spectral reflectance explains some of the features of color that have seemed difficult to reconcile with an objectivist account of colour. The causal irrelevance of color... can now be understood, as well as the fact that this lack of causal power is consistent with the objectivity of color. [...] Reflectance is not among the fundamental properties physical scientists use in explaining phenomena. Instead it is a dispositional property which, although not identical with any particular physical constitution, is well understood in terms of the fundamental properties of physics.“ (Hilbert (1987): 120) 
Gerade Hilbert betont den dispositionalistischen Charakter; er wird zum Beispiel aber auch von Broackes hervorgehoben ((1992): 456). Es stellt sich jedoch sofort die Frage, über welche Wirkungen die einzelnen Disjunkte der Basismenge einer Farbdisposition aktual individuiert werden sollen. Im Grunde sind drei Hauptvarianten einer solchen Position denkbar (oder wenigstens in der Literatur vorfindlich): (i) Dispositionen zur Beeinflussung von Licht; (ii) Dispositionen zur Hervorrufung von bestimmten Zuständen des visuellen Systemes eines Subjektes; (iii) Dispositionen zur Hervorrufung von phänomenal individuierter Farbwahrnehmungen. Nun stellt es sich aber heraus, daß faktisch alle drei dispositionalen Bestimmungen zu den denselben Reflektanztypen führen werden. Das heißt, alle drei Varianten ordnen dieselben Basiseigenschaften den betreffenden Farbdispositionen zu. Die dritte Alternative ergibt sich automatisch aus der Gültigkeit von $\left(\mathrm{ACT}_{\mathrm{NB}}\right)$, die beiden anderen entsprechen den empirischen Erkenntnissen über die naturwissenschaftlichen Grundlagen des Farbensehens (andernfalls kämen diese Alternativen erst gar nicht für eine Theoretisierung der Natur der Farben in Frage). Wenn man nun die Reflektanztypen mit den Farbdispositionen, und diese wiederum mit den entsprechenden Basismengen gleichsetzt, dann ergibt sich, daß alle drei Optionen ineinsfallen.

Für den objektivistischen Dispositionalismus ist diese Konsequenz sogar wünschenswert, weil es sich so einfach ergibt, daß Farben objektive Eigenschaften sind: da die einzelnen Disjunkte - die Reflektanzprofile - eine objektive Natur haben, gilt dies natürlich auch für die aus diesen gebildeten Disjunktionen. Der subjektivistische Dispositionalismus muß dagegen aus demselben Grund diese Folgerung auf jeden Fall vermeiden; sonst wären Farben eben keine subjektiven Eigenschaften. Er kann dies dadurch versuchen, indem er die dritte der Charakterisierungen als eine Analyse der Natur der Farben annimmt, so daß die korrespondierende Dispositionalität hinsichtlich der mentalen Zustände diesen nicht nur akzidentiell, sondern wesentlich zukommt. Doch diese Bestimmung kann für ihn nur Wert haben, wenn er zudem auch noch die Gleichsetzung von den Farbdispositionen mit den objektiven Disjunktionen aus Basiseigenschaften vermeiden kann. Dafür wird er wohl Dispositionen als genuine Universalien akzeptieren müssen - mit all den damit verbundenen Problemen. Ob der subjektivistische Dispositionalismus demnach überhaupt noch eine angemessene 
Theorie der Farben sein kann, soll jedoch erst im Subjektivismuskapitel geklärt werden. Hier sei einfach eine solche Position als gegeben vorausgesetzt.

Da der Subjektivismus nun eine Geistabhängigkeit der Farbtatsachen behauptet, steht ihm eigentlich nur die dritte Wirkungsart für die Farbdispositionen zur Auswahl. Die folgenden, als metaphysische Notwendigkeiten formulierten Bestimmungen des mit dem reinen Rotton identifizierten Reflektanztypes $S S R_{\mathrm{ROT}}$ zeigen dementsprechend auch ganz deutlich die Ähnlichkeit der ersten beiden Optionen $\left(D_{L}\right)$ und $\left(D_{v S}\right)$ und deren Verschiedenheit von der alternativen, dritten Charakterisierung $\left(D_{M}\right)$ :

$\left(\mathrm{D}_{\mathrm{L}}\right) \quad S S R_{\mathrm{ROT}} \equiv$ die Disposition, unter Normalbedingungen einfallendes Licht so zu beeinflussen, daß mehr Licht im lang- als im mittelwelligen Bereich reflektiert wird, und im kurzwelligen etwa soviel wie in den beiden anderen zusammen $(L>M ; S » L+M$; wobei $L, M$ und $S$ die Gesamtintensitäten des Lichtes in den entsprechenden Wellenlängenabschnitten bezeichnen).

$\left(\mathrm{D}_{\mathrm{VS}}\right) \quad S S R_{\mathrm{ROT}} \equiv$ die Disposition, unter Normalbedingungen in einem visuellen System, welches über drei Rezeptorarten verfügt und in seiner Verarbeitung der eingehenden Reize der Gegenfarbtheorie genügt, einen Zustand der chromatischen Informationskanäle hervorzurufen, der wie folgt beschrieben werden kann: $L>M ; S » L$ $+M$ (wobei $L, M$ und $S$ die registrierten Gesamtintensitäten des von den Rezeptoren aufgenommenen Lichtes in den entsprechenden Wellenlängenabschnitten bezeichnen). Bei diesen so kodierten Zuständen handelt es sich dabei um die Output-Zustände des visuellen Systemes.

$\left(\mathrm{D}_{\mathrm{M}}\right) \quad S S R_{\mathrm{ROT}} \equiv$ die Disposition, unter Normalbedingungen in einem Subjekt $S$ eine Farbwahrnehmung mit einem mentalen Rotquale hervorzurufen.

Der einzige Unterschied zwischen $\left(D_{L}\right)$ und $\left(D_{v S}\right)$ betrifft die Einbeziehung eines visuellen Systemes, welche natürlich auch mit der entsprechende Er- 
weiterung der relevanten Normalbedingungen einhergeht. Doch beide werden für uns als Farben erst deswegen interessant, da sie aktual unter Normalbedingungen mit Farbwahrnehmungstypen korreliert sind; und sie beziehen sich gleichermaßen wesentlich auf die Beschaffenheiten der für Farbensehen in Frage kommenden Subjekte: weswegen sie beide subjektrelativ zu nennen sind. Auch für $\left(\mathrm{D}_{\mathrm{L}}\right)$ läßt sich die Einschränkung auf drei Wellenlängenbereiche, die zudem den Bereichen $L, M$ und $S$ gleichen und in denen die Intensitäten der Sensitivitätskurve menschlicher Augen entsprechend addiert werden, keine angemessene Erklärung finden, wenn nicht auf das in $\left(\mathrm{D}_{\mathrm{vS}}\right)$ erwähnte visuelle System explizit Bezug genommen wird. In diesem Sinne ist es eigentlich gleichgültig, welche der beiden Optionen gewählt wird. Dementsprechend werden sie im weiteren Verlauf als gleichwertig angesehen werden. Für den Objektivisten fallen sie sowieso zusammen, da für ihn allein die aktualen, disjunktiven Basismengen interessant sind, die für $\left(D_{L}\right)$ und $\left(D_{V S}\right)$ identisch sind. Dabei soll jedoch eine wichtige Asymmetrie nicht außer acht gelassen werden. Denn es gibt Welten, in denen ein Gegenstand eine $\left(\mathrm{D}_{\mathrm{L}}\right)$-Disposition manifestieren kann, aber nicht die entsprechende $\left(\mathrm{D}_{\mathrm{vS}}\right)$-Disposition. Dies ist genau dann der Fall, wenn dort der Übergang von der Lichtbeschaffenheit zu den Informationskanälen im visuellen System nicht naturgesetzmäßig gewährleistet ist.

$\mathrm{Da}$ es sich bei der Subjektrealitivität nicht um eine Subjektabhängigkeit handelt, ist im Fall von $\left(\mathrm{D}_{\mathrm{L}}\right)$ relativ einsichtig. Dementsprechend sollte auch für $\left(\mathrm{D}_{\mathrm{vS}}\right)$ gelten, daß die so bestimmten Dispositionen zwar kontingenterweise über Subjektbeschaffenheiten individuiert werden, aber nicht geistabhängig sind. Die fraglichen Zustände des visuellen Systemes sind natürlich keine mentalen Zustände; ebenso läßt es sich vermuten, daß sie keine mentalen Zustände realisieren, sondern sich nur in Kausalketten befinden, die zu Wahrnehmungszuständen führen. Dahinter steckt die Idee, daß der Geist sich der Ergebnisse des visuellen Systemes zwar bedient, aber erst ,hinter“ diesem beginnt. Es macht nun wenig Sinn, bei der derzeitigen Erkenntnislage der Neurophysiologie darüber zu spekulieren, ob Mentalität auf Physikalität reduziert werden kann oder ob es bei einer irgendwie beschaffenen Realisation bleibt; und auch darüber, welche Gehirnzustände jeweils dabei involviert sind, und inwiefern das visuelle System dazuzurechnen ist. Genauso unbestimmt ist derzeit auch noch die neurophysiologische Manifestation der beiden chromatischen Informati- 
onskanäle, die die Gegenfarbtheorie postuliert und die als die für die nach $\left(\mathrm{D}_{\mathrm{vS}}\right)$ bestimmten Farbdispositionen relevanten Output-Zustände des visuellen Systemes angesehen werden können. ${ }^{22}$

Trotzdem soll hier die Hypothese vertreten werden, daß die Realisierung eines visuellen Systemes einschließlich der Informationskanäle nicht an einen Geist gebunden ist. Denn das visuelle System läßt sich vollständig physikalisch oder funktional beschreiben, ohne daß auf mentale Zustände zurückgegriffen werden müßte. Das heißt, auch eine Maschine sollte aufgrund von Rezeptoren mit derselben Sensitivität des menschlichen Auges sowie einer der menschlichen entsprechenden Weiterverarbeitung der eingegangenen Signale prinzipiell in der Lage sein, zu den gleichen Klassifikationen von Gegenständen aufgrund ihrer Reflektanzvermögen zu gelangen. Die funktionale Beschreibung der Gegenfarbtheorie träfe dann ebenfalls auf diese Maschine zu - nur mit der offensichtlichen Einschränkung, daß die Ausgaben nicht über phänomenale oder mentale, sondern über rein maschinelle Zustände erfolgen würden. Solche Maschinen können ohne weiteres hergestellt werden, kennt man in etwa die quantitative Kodierung der Gegenfarbtheorie. Die Maschine könnte dabei sogar Farben repräsentieren, wenn sie diese Funktionalität von ihrem Konstruktor zugewiesen bekäme. Deswegen ist es für einen funktionalen Zustand, der zum Beispiel Rot kodiert (und zwar wie folgt: $L>M ; S » L+M$ ) und somit repräsentiert, kontingent, ob er zu einer solchen Maschine oder zu einem Subjekt mit einem Geist gehört. Trotzdem wird er nur als Farben detektierender Zustand gelten, sobald er in bestimmten Subjekten vorkommen und nomologisch mit Farbwahrnehmungen verbunden sein könnte. In diesem Sinne gilt auch die Maschine nur dann als farbendetektierende, wenn sie einem visuellen System eines farbwahrnehmungen Subjektes funktional gleicht (vgl. Smart (1975): 57; Averill (1982): 19).

Dieses Maschinenbeispiel zeigt wieder einmal, daß die These (IND) jeder Farbtheorie zugrundeliegen muß: denn warum eine Eigenschaft als

22 Es gibt natürlich im Grunde beliebig viele Möglichkeiten, $\left(\mathrm{D}_{\mathrm{vs}}\right) \mathrm{zu}$ formulieren. Zum Beispiel könnten auch die proximalen Reizungen der drei Rezeptorarten oder bestimmte Zustände im optischen Nerv als relevant identifiziert werden solange es sich eben nicht um mentale oder solche realisierende Zustände handelt. Für die folgende Diskussion reicht jedoch die prinzipielle Betrachtung des im Text gemachten Vorschlages, der zudem eine gewisse Plausibilität aufweist. 
Farbe gelten sollte, kann letztlich nur durch einen engen Zusammenhang zu unseren Farbwahrnehmungen plausibel gemacht werden. Der nichtphysikalistische Dispositionalist, der die Analyse $\left(D_{M}\right)$ bevorzugt, interpretiert diese Grundintuition so stark, daß eine ontologische Geistesabhängigkeit der Farbtatsachen das Resultat ist. Die physikalistischen Dispositionalisten hingegen, ob sie nun $\left(D_{L}\right)$ oder $\left(D_{v S}\right)$ wählen, müssen nur eine Subjektrelativität der Individuation der Klasse der Farbtatsachen oder -eigenschaften annehmen, da sie sich zwar auf visuelle Systeme von Subjekten beziehen, diese visuellen Systeme aber nur kontingenterweise mit Lebewesen verbunden sind und dementsprechend eine objektive Beschreibbarkeit zeigen. In beiden Fällen ist es zwar wichtig, daß die Dispositionen als Farbdispositionen gelten, weil sie in der aktualen Welt unter Normalbedingungen mit phänomenal spezifizierten Farbwahrnehmungstypen nomologisch korreliert sind; aber nur laut dem subjektivistischen Dispositionalismus ist dies den Farben nicht bloß akzidentiell, sondern wesentlich: und es liegt eine Geistabhängigkeit vor. Für den subjektrelativen oder anthropozentrischen Dispositionalismus gilt dies nicht:

„Anthroporelativism carries no metaphysical punch“ (K. Campbell (1993): 251).

Die eben getroffene Unterscheidung ist damit genau dieselbe, welche eingangs bereits zwischen einer ontologischen Subjektabhängigkeit und einer nicht-ontologischen Subjektrelativität getroffen worden ist. Vor diesem Hintergrund läßt sich die Differenz zwischen dem objektivistischen und dem subjektivistischen Dispositionalismus auch auf das Bikonditional (BK) übertragen: während $\left(\mathrm{D}_{\mathrm{M}}\right)$ die epistemische Notwendigkeit garantiert und die Begründungsrichtung von rechts nach links laufen läßt, gilt dies für die beiden anderen Analysen der Rotdisposition - $\left(D_{L}\right)$ und $\left(D_{V S}\right)$ - nicht, so daß die Begründungsrichtung von links nach rechts zeigt. Nun ist dem objektivistischen Farbdispositionalismus üblicherweise vorgeworfen worden, weder Platz für die Disjunktivität oder die Anthropozentrik, noch für die kausale Unwirksamkeit von Dispositionen bieten zu können (vgl. etwa Armstrong (1987): 8ff.). Zwei der Merkmale sind nun von dem Verdacht befreit worden, ein Hindernis dafür zu sein, auf der Basis von solchen Dispositionen eine physikalistische Theorie aufzubauen: die Disjunktivität und 
die Subjekt-Relativität genügen ohne weiteres dem Anspruch der Objektivität. Doch es bleibt der Vorwurf, daß die Epiphänomenalität und, vor allem, das Fehlen einer Erklärungskraft mit einem Physikalismus nicht vereinbar sind. Doch der Schwache Physikalismus - in der Form des von Hilbert so benannten anthropocentric realism - hat gute Aussichten, auch dieses Problem zu lösen (vgl. Hilbert (1987): 15).

\subsubsection{Epiphänomenalismus und Erklärungskraft}

Dispositionen (und Disjunktionen) sind als Eigenschaften zweiter Ordnung kausal unwirksam. Stattdessen zeigen nur die entsprechenden, physik-immanenten Supervenienzbasen einen kausalen Einfluß auf andere, wiederum physik-immanente Tatsachen in der Realität. Farben als Reflektanztypen verstanden sind damit - ganz unabhängig davon, über welche Art von Wirkung sie definiert sind - ebenfalls immer epiphänomenal. Nun ist aber die Erklärungskraft ein Teil des repräsentationalen Aspektes der Intuitiven Farbkonzeption, so daß der Physikalismus, welcher per definitionem von der systematischen Veridizität von Farbwahrnehmungen ausgeht, auch diese für Farben aufrechterhalten muß. Zudem könnten Farben sonst aus ontologischer Sicht als überflüssig und ohne angesehen werden. Dahinter verbirgt sich wiederum nichts anderes als das Hauptproblem für die sekundären Qualitäten: einerseits werden sie von uns als ein fester Bestandteil der Welt angesehen, andererseits finden sie angeblich jedoch keinen Platz in unseren naturwissenschaftlichen Beschreibungen derselben. Dieser Vorwurf ist in dem Sinne von größter Bedeutsamkeit, da er nicht nur vom Objektivismus beantwortet werden muß, sondern generell von allen ontologischen Positionen, die Instantiierungen von Farbeigenschaften als existent annehmen wollen.

„The core of the complaint againts colours, as is well known, is their apparent failure to take a working role in the world's causal net. They seem not to belong, as G.F. Stout so memorably put it, to the 'executive order of nature'... Accepting that, and accepting the Platonic notion that power is the mark of being, we seem to be left with the conclusion that the colours have no being, at least in the causal nexus of Nature. That is the metaphysical problem about colour.“ (K. Campbell (1993): 250) 
Die verschiedenen philosophischen Theorien der Farben versuchen, diesem Problem entweder Tribut zu zollen, indem sie die Existenz von Farbtatsachen leugnen, oder ihm bei der Annahme solcher Tatsachen auf unterschiedlichste Weisen zu entgehen. Der eliminative Projektivismus wählt den ersten, radikaleren Weg. Der Starke Physikalismus löst das Problem dadurch auf, daß er Farben mit kausal wirksamen, nämlich physik-immanenten, Eigenschaften gleichsetzt. Die dispositionalistischen Varianten - ob objektiv oder subjektiv - hoffen dagegen auf eine nicht-kausale Erklärungskraft. Trotz ihrer kausalen Unwirksamkeit gelten Dispositionen jeglicher Art immer noch als Kräfte (powers); und sobald sich hierfür eigene, nicht-kausale Gesetzmäßigkeiten finden lassen, können diese Kräfte trotz allem als (wenn vielleicht auch nur supervenierende) Bestandteile der Realität angesehen werden - was jedoch nicht so einfach zu sein scheint, vor dem Hintergrund der Erkenntnis, daß dispositionale Gesetze tautologischen Charakter haben und Dispositionen deshalb nicht das (mögliche) Auftreten ihrer Manifestationen erklären können.

Auch der Schwache Physikalismus muß also noch den Beweis für die „Daseinsberechtigung“ der Farbdispositionen antreten. Für einen objektivistischen Dispositionalismus ergibt sich eine Erklärungskraft der Farben einfach aus dem Umstand, daß Farben repräsentierte Eigenschaften sind. Denn damit stehen sie, wenigstens faktisch und unter Normalbedingungen, in einem nomologischen Verhältnis zu den entsprechenden Farbwahrnehmungstypen. Farbtatsachen können somit das Auftreten von Farbwahrnehmungen erklären. Genau dies ist in der Umschreibung ausgedrückt, daß die Begründungsrichtung des Bikonditionals (BK) von links nach rechts verläuft (und dies gilt für alle Formen des Objektivismus). Eine Person hat normalerweise eine Rotwahrnehmung, weil es vor ihr in der Außenwelt etwas objektiv Rotes gibt, daß sie gerade wahrnimmt. Es gibt dementsprechend Gesetze, die Farben zutreffenderweise als subjekt-unabhängige Explananda für bestimmte Wirkungen in Subjekten ansehen. Dabei muß es sich natürlich nicht um Kausalgesetze handeln, da Repräsentation gerade nicht unbedingt mit einer kausal-nomologischen Korrelation gleichzusetzen ist. In dem Abschnitt über Dispositionen ist zwar mehrmals darauf hingewiesen worden, daß Dispositionen keine Erklärungskraft hinsichtlich ihrer Manifestationen haben; aber sie können, je nach gegebenen Naturge- 
setzmäßigkeiten, bestimmte, kausale Wirkungen ihrer Manifestationen erklären. Ein Objektivismus bringt demnach wirklich immer eine Erklärungskraft der Farben mit, da er diese als repräsentierte Eigenschaften analysiert. Und insbesondere der Schwache Physikalismus kann systematische Veridizität gewährleisten.

\subsubsection{Farbmultiplizität und Naturgesetzmäßigkeiten}

Auf zwei wichtige Konsequenz des Schwachen Physikalismus muß noch hingewiesen werden. Zuerst einmal besteht der Umstand, daß entweder nur Menschen, wie sie aktual beschaffen sind, Farben wahrnehmen können, oder daß jeder Gegenstand mit Reflektanzeigenschaften im Prinzip beliebig viele Farben gleichzeitig haben kann und somit multipel gefärbt ist. Denn Lebewesen, die ein visuelles System aufweisen, welches von dem unseren in einigen Punkten abweicht (etwa der Anzahl oder der Sensitivität der Rezeptorenarten), werden natürlich auch andere Reflektanzvermögen der Gegenstände repräsentieren und diesen somit ganz andere Farben zuschreiben. Die Subjektrelativität geht also soweit, daß ein- und dasselbe Reflektanzprofil von verschiedenen Subjektgruppen in ganz unterschiedliche Klassen geordnet (oder unter verschiedene Typen gefaßt) wird. Zwei Gegenstände, die für Menschen gleichfarbig aussehen, können für bestimmte Tiere verschiedenfarbig sein. In diesem Sinne gibt es hinsichtlich jeder farbenwahrnehmenden Spezies eine eigene Farbdeterminable und eine eigene Menge von Farbdeterminanten. ${ }^{23}$ Man hat nun die Wahl, nur Menschen oder auch andere Spezies von Lebewesen als farbwahrnehmend einzustufen. Innerhalb der Biologie angestellte Überlegungen hierzu machen es plausibel, die letztere Alternative zu wählen: wenigstens Primaten und anderen, höherentwickelten Säugetieren sprechen die Biologen phäno-

23 Averill gibt zusätzlich hypothetische Fälle von menschen-ähnlichen Lebewesen an, die Gegenstände in andere Farbklassen als Menschen einteilen, weil zum Beispiel bestimmte filter-ähnliche Gebilde Teil ihrer Augen sind, die zum Beispiel Inversionen herbeiführen, hinsichtlich der Polarisation des eingehenden Lichtes empfindlich sind oder auch für verschiedene Blickrichtungen Unterschiede machen (vgl. Averill (1982): 23f.). Der Umstand, daß Gegenstände für verschiedene Subjekte unterschiedlich gefärbt sein können, bewegt Averill dazu, die SubjektRelativität aufzugeben und einen Starken Physikalismus anzunehmen. 
menales Farbensehen zu. Aber damit wesentlich verbunden ist eben die Annahme, daß ein Gegenstand für Menschen die eine, und für bestimmte Tiere eine ganz andere Farbe instantiieren kann. Bienen oder Hunde sehen Blumen zum Beispiel in ganz anderen Farbtönen als wir. Farben können somit als Klassifizierungsmerkmale aufgefaßt werden, mithilfe derer Objekte aufgrund ihrer Eigenschaften erster Ordnungen und deren normalen Wirkungen auf Subjekte einer bestimmten Spezies in verschiedene, subjekt- oder speziesrelative Farbklassen zusammengefaßt werden. ${ }^{24}$

Hier mag nun der Verdacht aufkommen, daß es sich doch um einen Farbsubjektivismus handelt. Aber diese Befürchtung ist unbegründet, da die Physikalität der Farben erhalten bleibt und die Subjektrelativität keine ontologische Geistabhängigkeit beinhaltet. Nur die Relevanz der Farbeigenschaften - warum sie überhaupt Teil einer objektiven Beschreibung der Welt sein sollen - hängt dem Schwachen Physikalismus zufolge davon ab, daß sie über visuelle Systeme analysiert werden, die kontingenterweise mit bestimmten Subjekten verbunden sind. Insbesondere wird es für alle subjektrelativ bestimmten Mengen von Farbdeterminanten und - determinablen möglich sein, eine allgemeine Gemeinsamkeit festzuhalten: sie bilden jeweils eine besondere Untermenge der physikalischen Art der Reflektanztypen-an-sich. Zudem gilt auch dann noch für alle Farben die grundsätzliche These $\left(\mathrm{IND}_{\mathrm{R}}\right)$ : von Subjekten in Farbwahrnehmungen repräsentiert zu werden. Und die Rigidizität, auf den Menschen bezogen, kann ohne weiteres erhalten bleiben: Veränderungen der Farbigkeit sind allein auf Veränderungen der intrinsischen Beschaffenheiten der Gegenstände zurückzuführen. Trotzdem zeigt der Starke Physikalismus natürlich immer noch den Vorteil, daß er für alle farbwahrnehmenden Subjekte - ganz gleich, welcher Spezies sie zugehören - ein- und dieselbe physikalische Art als Far-

24 Hilbert behauptet eine ähnliche Subjekt-Relativität auch für die Klassifikationen von Formeigenschaften (vgl. Hilbert (1987): 75ff.). Doch die obigen Ausführungen über die Asymmetrie zwischen Farben und Formen haben gezeigt, daß die subjekt-relative Klassifikation letzterer im Grunde wie eine physik-immanente funktioniert, wenn man zum Beispiel ein Längenmeßgerät verwendet, welches Längenunterschiede ähnlich genau wie das menschliche Auge feststellen kann. Trotzdem hat Hilbert weiterhin Recht mit seiner Behauptung, daß die SubjektRelativität den Objektivitätscharakter nicht verletzt: sie hat keinen Einfluß auf den ontologischen Status der Farbeigenschaften. 
ben annehmen kann: er kann - im Gegensatz zum Schwachen Physikalismus - dieser spezies-relativen Multiplizität der Farbigkeit von Gegenständen aus dem Wege gehen.

Der zweite Punkt ist, daß der Bestand der für die Manifestierung von Farbdispositionen mitverantwortlichen Naturgesetzmäßigkeiten einen wesentlichen Bestandteil der Normalbedingungen für Farbwahrnehmungen ausmachen wird, sobald man einen objektivistischen Dispositionalismus akzeptiert. Betrachten wir einen Gegenstand, der aufgrund seines Reflektantprofiles $S S R$ die Reflektanzdisposition $S S R_{\mathrm{ROT}}$ aufweist, im menschlichen visuellen System unter Normalbedingungen die entsprechende Rotkodierung $V S_{\text {ROт }}$ hervorzurufen: dem Schwachen Physikalismus zufolge ist er damit rot. Verändern sich die Naturgesetzmäßigkeiten jedoch so, daß SSR auf einmal mit der Kodierung $V S_{\text {GRÜN }}$ nomologisch verbunden ist, dann wird der Gegenstand für Menschen unter Normalbedingungen (wenn diese unverändert bleiben) nicht mehr rot, sondern grün aus. Kommt es dagegen durch eine nochmalige Veränderung der Naturgesetzmäßigkeiten dazu, daß SSR gar keinen Einfluß mehr auf das menschliche visuelle System nehmen kann, dann verliert der Gegenstand für uns sogar seine sichtbare Farbigkeit ganz. Will man nun eine ontologische Abhängigkeit der Farbtatsachen von den betreffenden Naturgesetzmäßigkeiten vermeiden, dann bietet es sich an, die in der aktualen Welt für die Spezifizierung von Farbdispositionen erforderlichen Naturgesetzmäßigkeiten jeweils in die entsprechenden Normalbedingungen miteinzuschließen.

Damit führen die Veränderungen der vorherrschenden Naturgesetze dazu, daß zwar die Farbdispositionen weiterhin instantiiert sein können, aber daß die für ihre korrekte Wahrnehmung benötigten Normalbedingungen nicht mehr eintreten können: die Manifestierung der fraglichen Dispositionen ist nichr mehr möglich. Damit bleibt die Rigidizität gewährleistet, da Farbtatsachen nur auf den Tatsachen über die intrinsischen Eigenschaften der Objekte supervenieren. Doch die Normalbedingungen müssen auf einmal farbton-spezifische Elemente beinhalten: denn die einzelnen Farben wird natürlich jeweils eine andere Naturgesetzmäßigkeit mit den entsprechenden Farbwahrnehmungen verbinden. Aber dies ist eigentlich auch nicht überraschend, da jede einzelne Disposition ihre eigenen Manifestierungsbedingungen aufweist: dies gilt für Farbdispositionen wie auch für alle anderen, multipel realisierbare Dispositionen (die Löslichkeit von Salz 
in Wasser benötigt zum Beispiel auch ganz andere Naturgesetzmäßigkeiten zur Manifestierung als die entsprechende Löslichkeit von Zucker). Ganz abgesehen davon begegnen wir hier wieder einer Schwierigkeit für den subjektivistischen Dispositionalismus. Da dieser die Rigidizität vermeiden und dem Gegenstand jeweils die Farbeigenschaft zuschreiben möchte, die dieser in der betreffenden, beliebigen Welt typischerweise zeigt, wird er die Normalbedingungen für Farbwahrnehmungen ohne Einbeziehung irgendwelcher Naturgesetzmäßigkeiten angeben. Damit läßt sich aber eine Supervenienz der Dispositionen auf den jeweiligen Naturgesetzmäßigkeiten nur dadurch verhindern, daß Dispositionen als genuine Universalien angesehen werden. Daß dies aber $\mathrm{zu}$ enormen Schwierigkeiten führt, soll der Abschnitt über den subjektivistiscne Dispositionalismus zeigen. Jedenfalls wird dem Schwachen Physikalismus sein objektivistischer Status und seine Plausibilität als eine Theorie der Farben belassen.

\subsection{Starker und Schwacher Physikalismus im Ver- gleich}

Der physikalistische Objektivismus besteht also aus zwei großen Varianten: dem Starken und dem Schwachen Physikalismus. Während die erste Position Farben mit physik-immanenten Reflektanzprofilen gleichsetzen möchte, versucht letztere dies in Hinsicht auf als Dispositionen oder Disjunktionen zu verstehende Reflektanztypen. Beide werden als objektivistische Positionen dem repräsentationalen Aspekt der Intuitiven Farbkonzeption gerecht: die These (REP) besitzt Gültigkeit. Der Starke Physikalismus kann Farben als intrinsische, physik-immanente Eigenschaften mit kausaler Wirksamkeit bestimmen. Dafür muß er jedoch von empirisch bestimmbaren Gehaltsunterschieden unterhalb der Sensitivität des Auges ausgehen, die somit nicht mehr introspektiv zugänglich sind. Da damit auch die Aufgabe oder Modifkation der These (ACT) verbunden ist, wird ein stark-physikalistischer Objektivismus $S_{\mathrm{NEC}}$ nicht mehr - oder nur noch sehr eingeschänkt - möglich sein. Der Schwache Physikalismus hingegen sieht Farben nur als subjekt-relativ individuierte Eigenschaften zweiter Ordnung an, 
die auf den intrinsischen Beschaffenheiten der betreffenden Gegenstände supervenieren. Die Hauptnachteile für eine solche Position sind zum einen der ontologische Status - daß Farbtatsachen über ihre physik-immanenten Realisatoren kein Mehr in der Realität bedeuten und Farben damit keine genuinen Merkmale in der Welt darstellen - und zum anderen die damit verbundene Epiphänomenalität (trotz vorhandener, nicht-kausaler Erklärungskraft): Auch die spezies-relative Multiplizität von Farbeigenschaften in der Welt ist keine unbedingt wünschenswerte Charakteristik.

Beide Alternativen haben also ihre gewichtigen Vor- und Nachteile. Der Starke Physikalismus nimmt das faktische Auseinandergehen von repräsentationalen und phänomenalen Differenzen, der Schwache andererseits die Dispositionalität mit all ihren problematischen Merkmalen in Kauf, um die für den Objektivismus so entscheidende These (REP) aufrechterhalten zu können. Im Gegensatz dazu muß ein Formphysikalismus keine solchen Konzessionen machen, da die repräsentierten Klassen von Formeigenschaften wiederum physikalische Arten bilden. Die zwei vorgestellten Alternativen des Farbphysikalismus versuchen also, diesen Formphysikalimus für Farben so weit wie möglich zu retten, indem sie jeweils auf verschiedene Merkmale verzichten. Trotzdem handelt es sich im Grunde bei dem Starken und dem Schwachen Physikalismus um zwei Seiten ein- und derselben Theorie. Wie eng diese beiden Alternativen jedoch wirklich zusammenhängen könnten, sollen die folgenden Überlegungen am Beispiel der Positionen von Jackson, Smart und Armstrong andeuten. Dabei spielt insbesondere der Umstand eine bedeutsame Rolle, daß Physikalisten hinsichtlich von Farben auch dazu neigen, eine vergleichsweise physikalistische oder kategoriale Theorie der Dispositionen und Disjunktionen anzunehmen, die die Anwendbarkeit des ontological free lunch auf diese Eigenschaftsarten beinhaltet.

Jackson unterscheidet strikt zwischen Dispositionen und deren Basiseigenschaften: seine weiten Dispositionen entsprechen vielmehr den betreffenden, disjunkten Basismengen und sind als solche eindeutig kausal unwirksam. Dem Umstand, daß er - fälschlicherweise, wie die Überlegungen von Lewis mit Bezug auf Wahrnehmungen gezeigt haben - das Bestehen einer kausalen Nomologizität als notwendige Bedingung für das Bestehen einer Repräsentationsrelation ansieht, ist es zu verdanken, daß er die epi- 
phänomenalen Dispositionen nicht als die repräsentierten Farben ansehen kann (oder möchte), sondern vielmehr die einzelnen Basiseigenschaften mit diesen identifiziert. Damit ist er ein Starker Physikalist und muß die Aktualitätsthese (ACT) aufgeben. ${ }^{25}$ Interessanterweise nimmt Jackson, um die Einheitlichkeit der normalerweise als gleichfarbig gesehenen Basiseigenschaften $\mathrm{zu}$ retten, auf Reflektanztypen Bezug, ohne diese jedoch mit den Farben wirklich gleichzusetzen.

„Nevertheless, part of the story is relatively uncontroversial. A necessary condition for $\mathrm{E}$ to be the presentation of $\mathrm{P}$ is that there be a causal connection. [...] We can work with the rough schema: redness is the property of objects which typically causes them to look red in the right way. [...] The dispositional theory of color is mistaken because dispositions are not causes, and in particular not causes of their manifestations. Their categorial bases do all the causing. [...]

It follows, therefore, from the prime intuition that the colors are presented in color experience and so are causes or potential causes of things' looking one or another color, that the colors are not dispositions to look colored. They are instead the categorical bases of dispositions to look colored. [...] The issue then in the case of color is whether there is a unifying distal property. Now there is some reason to hold that triples of reflectances correlate closely with perceived color. $[\ldots]$

25 Es passiert oft genug, daß Jackson und Pargetter (vielleicht nicht ganz zu unrecht) aufgrund ihres gemeinsamen Aufsatzes über Farben (1987) als Subjektivisten angesehen werden, da sie es dort als epistemisch notwendig ansehen, daß Farben Farbwahrnehmungen hervorrufen (vgl. Einleitung zu Byrne \& Hilbert (1997a): xxiii). Diese Einschätzung ist jedoch auf einen mißverständlichen oder ungeschickten Gebrauch des Begriffes der Apriorizität durch Jackson und Pargetter zurückzuführen, welcher sich auch in Jacksons Aufsatz ((1996): 208f.) wiederholt. Dort wird jedoch klar, daß es sich um eine objektivistische Position handelt, die Farben nicht wesentlich mit den von ihnen hervorgerufenen Farbwahrnehmungen zusammenhängen. Daß Rot unter Normalbedingungen Rotwahrnehmungen hervorruft, ist nur in der aktualen Welt wahr - aber damit auch notwendigerweise aktual wahr: in diesem Sinne kann hier von einer notwendigen Wahrheit geredet werden. Es besteht jedoch keine epistemische Notwendigkeit, so daß die folgende Behauptung nur bedingt zutrifft:

„The primary quality theory [of colors] can... honour the [subjectivistic] dispositionalist's insight that there is something a priori or truistic about the connection between being red and being appropriately disposed to look red.“(Jackson (1996): 208) 
Hilbert, as I understand him, thinks of the triples as themselves dispositional properties - as an object's disposition to reflect light displaying the relevant value of the triple. [...] But I cannot follow him in identifying the colors with these dispositions. We causalists must think of the value of the triple for a given color, red, say, as what unifies the possibly highly disjunctive basis that is responsible for the disposition to look red in normal circumstances." (Jackson (1996): 200ff.; 204; 215)

Auch wenn Jackson Farben nicht mit Reflektanztypen gleichsetzen möchte, bringt er sie jedoch mit diesen vermittels der Farbphänomenalität in einen engen Zusammenhang. Die Schwachen Physikalisten geben im Gegensatz die Forderung auf, daß Farben kausal wirksame Eigenschaften erster Ordnung sein müssen, da Repräsentierbarkeit dies nicht voraussetzt. Für sie bedeuten Farbtatsachen kein wirkliches Mehr zur bestehenden Realität; dafür können sie die aktuale Korrelation von Gehalts- und Qualiadifferenzen aufrechterhalten. Armstrong verteidigt so explizit Smarts Disjunktivismus (wenn auch immer noch mit der Hoffnung, daß Farben schließlich doch als physikalische Art konstruiiert werden können):

„Smart, Lewis and myself share a reductive (physicalistic) and realistic view of the secondary qualities." (Armstrong (1987): 13)

„The relative simplicity of the content of colour perception is to be explained as a failure to perceive all there is to perceive about the nature of these properties or states, including a failure to perceive their disjunctivity. The disjunctivity entails that colour is a 'second-class' property, that the colours are not universals." (Armstrong 1997): 58f.)

Doch die hier beschriebene Position kann sich sehr schnell verändern, sobald man die Natur der Eigschaften zweiter Ordnung genauer hinterfragt. Smart schweigt sich über dieses Thema aus. Lewis dagegen wendet sich anscheinend aufgrund der Disjunktivität und der damit verbundenen Epiphänomenalität von der Theorie ab (vgl. Armstrong (1987): 10). Aber auch Armstrong scheint sich ebenfalls von dem Schwachen Physikalismus zu entfernen, nimmt man seine Schriften über Dispositionen ernst und akzeptiert zudem die - bereits begründete - These, daß Dispositionen und Disjunktionen im Prinzip gleiche ontologische Gebilde darstellen. Damit wird Armstrongs Position über Farben jedoch alles andere als klar, wie es sich 
insbesondere in seinem neuesten Buch widerspiegelt. Farben werden dort, wie das eben gegebene Zitat zeigt, als Disjunktionen verstanden. Die einzelnen Disjunkte fügen sich jedoch zu der Disjunktion, also einer Eigenschaft zweiter Ordnung, nur deshalb zusammen, da sie unter Normalbedingungen Farbempfindungen ein- und desselben phänomenalen Types kausal hervorrufen (vgl. Armstrong (1987): 7; und Armstrong (1997): 58).

Damit ist das Farben wesentlich zukommende Merkmal dispositionaler Natur: Disjunktionen können als Dispositionen angesehen werden (und umgekehrt). Hinsichtlich von letzteren neigt Armstrong jedoch dazu, die Dispositionen mit den jeweiligen Basiseigenschaften - eben den Disjunkten - gleichzusetzen (vgl. Armstrong (1997): 73). Damit stände er aber im Gegensatz zu Smarts Analyse von Farben als Eigenschaften zweiter Ordnung. Sein Ansatz, Dispositionen auf Eigenschaften erster Ordnung zurückzuführen, legt nahe, daß auch die disjunktiven Farben solcherart auf ihre einzelnen Disjunkte reduziert werden können. Damit scheinen aber der Starke und der Schwache Physikalismus bei Armstrong zusammenzufallen - und auch bei Jackson ist diese Tendenz zu sehen (obwohl in der entgegengesetzten Richtung). Bei beiden verbirgt sich dahinter natürlich die Hoffnung, daß kausale Wirksamkeit (oder Physik-Immanenz) auf der einen Seite und Repräsentierbarkeit - einschließlich der Akzeptanz der Aktualitätsthese - auf der anderen Seite nicht nur in den Formeigenschaften, sondern letztlich auch in den Farbeigenschaften vereinigt werden können. Daß diese Hoffnung jedoch enttäuscht werden muß, haben die bisherigen Ausführungen gezeigt. Darin liegt auch der Grund für die Auftrennung der physikalistischen Position in zwei Theorien, die beide mehr oder weniger problematisch sind und zudem wie zwei Seiten ein- und derselben Sache erscheinen.

Doch gerade der vermeintliche Widerspruch in Armstrongs Texten hinsichtlich der Natur von Farben als Eigenschaften zweiter Ordnung läßt vermuten, daß im Grunde der Starke Physikalismus die vielversprechendere Position ist. Denn sie entgeht nicht nur der Epiphänomenalität und der Supervenienzabhängigkeit; sondern darüberhinaus scheint auch jeder ontologisch sinnvolle Weg, Dispositionen als Eigenschaften zu retten, auf deren Reduzierung auf die Basiseigenschaften hinauszulaufen. Sowohl Disjunktionen als auch Dispositionen werden über die möglichen Wirkungen ihrer 
Realisatoren individuiert. Die damit verbundenen Schwierigkeiten mit dem ontologischen Status legen nun eine Individuation über die Ursachen selbst, also die intrinsischen und physik-immanenten Eigenschaften, nahe, auch wenn damit die Aktualitätsthese und die Repräsentierbarkeit von Farben nur noch bedingt aufrechterhalten werden kann. Zudem kann die Objektivitätsintuition über Farben in ihrer vollen Stärke erhalten werden: Farben sind nicht mehr nur subjektrelative Klassifikationen von Gegenständen aufgrund ihrer Eigenschaften erster Ordnung, die für jede Spezies von farbwahrnehmenden Subjekten den Gegenständen unterschiedliche Farbeigenschaften zuschreibt; sondern Farben sind für alle solchen Spezies ein- und dieselben, da sie eine physikalische Art bilden.

Aber alle diese Vorteile können das fundamentale Problem des Starken Physikalismus nicht aufwiegen: wenn dieser die These aufgibt, daß Reflektanztypen repräsentiert werden, dann muß er gleichzeitig aufgeben, daß Farbwahrnehmungen überhaupt Eigenschaften repräsentieren. Denn für die Reflektanzprofile ist es, wenigstens nach dem bisherigen Stand der Dinge, nicht möglich, Normalbedingungen für die Repräsentation dieser Eigenschaften zu spezifizieren; und ohne Normalbedingungen kann keine Repräsentationsrelation zwischen den Farbwahrnehmungstypen und den für deren Auftreten kausal verantwortlichen Reflektanzprofilen Bestand haben. Es könnte sich höchstens um eine Detektionsrelation handeln; doch die Überlegungen zum Informationalismus haben auch dies als plausible Alternative ausgeschlossen. Dagegen sehen die Schwierigkeiten für den Schwachen Physikalismus doch ganz harmlos aus: die Epiphänomenalität wird durch die repräsentational begründete Erklärungskraft der Farbdispositionen ausgeglichen; der ontologische Status stellt mithilfe der These von ontological free lunch kein wirkliches Problem mehr dar; und die speziesrelative Multiplizität entspricht sogar den Intuitionen über das Farbensehen anderer Lebewesen. Demgemäß wird im folgenden der Schwache Physikalismus als eigentliche und beste physikalistische Position $\mathrm{zu}$ betrachten sein, derzufolge Farben mit den Reflektanztypen identisch sind. Damit unterscheiden sich aus ontologischer Sicht auch die primären von den sekundären Qualitäten. 


\subsection{Argumente gegen den Aktualitätsobjektivismus}

In den letzten Abschnitten sind bereits einige Probleme für die einzelnen Varianten der physikalistischen Position hinsichtlich von Farben aufgezeigt worden. Die meisten in der Literatur vorgebrachten Argumente richten sich jedoch auf den Starken und den Schwachen Physikalismus gleichermaßen. Die Einwände lassen sich dabei in zwei Gruppen teilen, je nach dem, ob sie den introspektiven Charakter von Farbwahrnehmungen, und damit auch die These der Transparenz, betreffen, oder ob sie sich auf bestimmte Erkenntnisse oder Ansichten über das menschliche Farbensehen beziehen. $\mathrm{Daß}$ der Physikalismus dem repräsentationalen Aspekt gerecht wird, ist ja bereits argumentiert worden und wird allgemein auch nicht angezweifelt. Ebenso ist der vermeintlich problematische Status von Normalbedingungen bereits untersucht worden und soll hier nicht noch einmal behandelt werden. Wie es sich zeigen wird, bietet die Akzeptanz der Notwendigkeitsthese $\left(\mathrm{NEC}_{\mathrm{NB}}\right)$ für den Physikalisten einen Ausweg aus den Hauptproblemen für seine Position, so daß die hier besprochenen Argumente im wesentlichen den Objektivismus ${ }_{\mathrm{ACT}}$ betreffen.

\subsubsection{Mit der Farbigkeit von Entitäten in der Welt verbunde- ne Einwände}

Beginnen wird mit den Gegenargumenten, die nicht unmittelbar mit der Intuitiven Farbkonzeption verbunden sind. Traditionellerweise wird gegen die Physikalisten eingewandt, daß sie nur die Farbigkeit einer sehr eingeschränkten Anzahl von Gegenständen erklären können: und zwar von undurchsichtigen, nicht-selbstleuchtenden Körpern (vgl. zum Beispiel K. Campbell (1979): 573). Doch es ist bereits in dem Kapitel über die Normalbedingungen daraufhingewiesen worden: daß, erstens, lichtdurchlässige Körper auf eine sehr ähnliche Weise wie lichtundurchlässige beschrieben und beide Beschreibungen im Grunde zu einer zusammengefaßt werden können (indem man Reflektanz- und Transmittanzphänomene mithilfe der Absorption als ein Gesamtphänomen auffaßt); und daß, zweitens, die Farbigkeit von Licht oder Lichtquellen ganz analog zu dem Fall der void 
viewing conditions betrachtet werden muß und somit streng genommen eigentlich eine Fehlrepräsentation darstellt, da das visuelle System bestimmte Informationen, die ihm $\mathrm{zu}$ einem Farbensehen noch fehlen, einfach „ergänzt“. Doch diese Konzession sollte einem Physikalisten keine Probleme bereiten, weil in den allermeisten Fällen von angeblich „farbigem Licht“ doch Reflektanz- oder Transmittanzvermögen eine Rolle spielen (vgl. Zeki (1993): Kap. 23): so kann das Blau, oder auch das Abendrot, des Himmels als eine Volumenfarbe aufgefaßt werden, jeweils in Abhängigkeit von den Bestandteilen und der Dichte der vom Sonnenlicht durchquerten Atmosphäre (vgl. Hardin (1988): 4); und Sonnen- oder auch Laserstrahlen werden oftmals deswegen anscheinend als farbig gesehen, weil sie auf bestimmte, in der Luft schwebende Partikel treffen (wie etwa Staub), wobei es sich hier im Prinzip um eine auf den Helson-Judd-Effekt zurückführbare Fehlrepräsentation der Farbigkeit der Partikel handelt. Hierunter fällt auch die von Hardin erwähnte kosmische Strahlung.

Dieser führt nun eine ganze Liste weiterer irregulärer Ursachen für Farbwahrnehmungen an (vgl. Hardin (1988): 91f.), die jedoch laut Farb physikalismus als Illusionen (oder Halluzinationen) erklärt werden können. So werden durch Drogen, Pressen des Augenballs, eine beginnende Migräne oder andere, vorstellbare Reizungen hervorgerufene Farbempfindungen nicht als veridisch gelten, da sie keinerlei Bezug zu einem wahrgenommenen Gegenstand aufweisen können, sondern vielmehr Formen von Halluzinationen sind. Dasselbe gilt für die Fähigkeit des visuellen Systemes, bestimmte Bereiche des Gesichtsfeldes auszufüllen, da diese aufgrund des Blinden Fleckes (dem Eintritt des optischen Nerves in die Retina, an dem selbst keine Rezeptoren liegen) sonst leer bleiben müßten. In diesem Bereich findet, wenn man so will, eine systematische Fehlrepräsentation statt, die jedoch durchaus ihren biologischen Nutzen hat, in den meisten Fällen auch nicht allzu stark von der Realität abweichen (zum Beispiel, wenn einigermaßen homogen gefärbte Flächen wahrgenommen werden) und nur selten wirklich bemerkt wird (da der blinde Fleck eher die Peripherie des Gesichtsfeldes betrifft und nur durch besondere Experimente ,sichtbar“" gemacht werden kann). Schließlich fallen auch die sogenannten instabilen, „schimmernden Farben“ in diese Kategorie: wie sie zum Beispiel auf Glanzeffekte zurückzuführen sowie auf Ölschlieren, $C D$ s oder auch in Ho- 
lographien zu sehen sind (vgl. Johnston (1992): 141). In all diesen Fällen ist es sehr einsichtig, eine Fehlerhaftigkeit der entsprechenden Wahrnehmungen anzunehmen: der Physikalismus wird diesem Umstand nicht nur gerecht, er setzt sich dadurch auch kaum einer Kontroverse aus.

Dies kann jedoch bei zwei anderen Effekten schon anders aussehen: dem Sukzessivkontrast sowie den sogenannten Phaseneffekten. Ein besonders bekanntes Beispiel für den Sukzessivkontrast sind die Nachbilder ( $a$ ter-images), die entstehen, wenn man zuerst lange eine farbige Fläche (oder auch farbiges Licht) betrachtet und dann auf einen beispielsweise weißen Grund schaut (oder, im Fall des Lichtes, einfach die Augen schließt). Der von Hardin beschriebene McCullough-Effekt kann als ein kognitiv höher angesiedelter Sukzessivkontrast angesehen werden. Beide lassen sich auf die begrenzte Reaktionszeit sowie die Adaptionsträgheit der Augenrezeptoren, der Zellen im optischen Nerv und der Zellen im visuellen Kortex zurückführen lassen (vgl. auch den Abschnitt über Normalbedingungen). Die Bidwell-Scheibe besteht zum Beispiel aus einer weißen und einer schwarzen Hälfte und hat eine kleine Aussparung an einer Seite, durch die ein unter ihr befindliches rotes Licht ab und zu sichtbar wird, wenn die Scheibe sich langsam dreht. Bei schnellerer Geschwindigkeit kann das Auge die Lampe nicht mehr erkennen, da es zu träge ist (wie auch das Signal auf einem Bildschirm als kontinuierliches gesehen wird). Stattdessen erscheint die Scheibe als blaugrün, also in der Komplementärfarbe von Rot: es liegt ein Nachbildphänomen vor (vgl. Hardin (1988): 92f.).

Der Phaseneffekt zeigt sich, wenn eine abwechselnd hell und dunkel gefärbte Fläche sich im Vergleich zum Auge auf eine bestimmte Weise bewegt. Wird die Benham-Scheibe, deren eine Hälfte schwarz und die andere weiß gestrichen ist, mit der richtigen Geschwindigkeit gedreht, werden Spuren von Farblinien auf ihr sichtbar (vgl. Hardin (1988). 72). Hinsichtlich beider Fälle ist das visuelle System offensichtlich nicht dafür entwickelt worden, die entsprechende Farbigkeit so zu sehen, wie es sie tut. Die Bewegung der betrachteten Objekte führt zu einer Farbillusion, da sie nicht als Bewegung erkannt wird: die Information über die jeweilige Positionierung der rotierenden Scheibe wird von der vom visuellen System selbst gelieferten Information über die vermeintliche Farbigkeit überdeckt. Somit können auch der Sukzessivkontrast und die Phaseneffekte ohne Probleme als Fehlrepräsentationen gelten: was auch durch ihre relative 
Seltenheit und Irrelevanz im alläglichen Umgang mit farbigen Objekten plausibel gemacht wird.

Ganz anders sieht es jedoch für den Simultankontrast hinsichtlich von Farbtönen oder Intensitäten aus (vgl. zum Beispiel Thompson (1995): 113f.). ${ }^{26} \mathrm{Da}$ es einen Farbtonkontrast gibt, leitet sich unmittelbar aus dem Umstand $a b$, daß Farbensehen einen zwischengegenständlichen Vergleichsprozeß miteinschließt, wie es etwa die Experimente von Edwin Land gezeigt haben (vgl. Zeki (1993): 230ff.). Da das visuelle System die Intensitäten der von verschiedenen Objekten ausgesandten Lichtwellen erst miteinander vergleichen muß, damit es daraus die benötigte Informationen über das Reflektanzvermögen herauslösen kann, ist es nur zu erwarten, daß Veränderungen in der farbigen Umgebung einer Fläche auch deren gesehene Farbigkeit beeinflussen können. Üblicherweise ist dieser Effekt jedoch so schwach oder wird durch eine größere Anzahl von Beeinflussungen ausgeglichen, daß er uns nicht auffällt. Nur bei besonderen Kontextanordnungen von Farbflächen wird er offensichtlich - und stellt somit ein Problem für den Physikalisten dar, da dieser Farben als intrinsische oder dispositionale Eigenschaften der Gegenstände für sich genommen ansieht. Der Physikalist kann nicht erklären, daß ein- und dieselbe Fläche vor dem einen Hintergrund anders aussieht als vor dem anderen, wenn sich die Reflektanzeigenschaften der Fläche nicht ändern und zudem Normalbedingungen vorliegen.

Der gleiche Einwand ergibt sich aus dem Umstand, daß erst der Intensitätskontrast das Sehen von Farbtönen wie Braun, Olivgrün oder Schwarz ermöglicht (vgl. K. Campbell (1993): 256f.). Gegenstände, die diese Farben für uns zeigen, weisen tatsächlich dieselben Reflektanzen auf, wie Gegenstände, die, je nach dem, als orange, grünlich-gelb oder dunkelgrau gesehen werden. Ganz deutlich wird dies insbesondere bei dem Vergleich von Schwarz und Weiß. Das Verhältnis der jeweils in den drei Wellenlängenbereichen reflektierten Lichtintensitäten ist bei beiden dasselbe (nämlich $L$ » $M » S$ ), aber die proportionalen Werte unterscheiden sich enorm (von wei-

26 Es gibt zudem noch einige andere Kontrastphänomene, die jedoch hier unberücksichtigt bleiben, da sie entweder analog wie der Simultankontrast erklärt oder wie der Sukzessivkontrast als illusionär betrachtet werden können. Eine ausführliche Beschreibung dieser Phänomene findet sich in Albers (1975) und, vor allem, in Itten (1983). 
ßen Gegenständen werden etwa $90 \%$, von schwarzen jedoch nur etwa $4 \%$ Licht reflektiert). So kommt es, daß ein Stück Kohle in der prallen Sonne zwar mehr Licht ausstrahlt als ein weißes Papier im Schatten, aber trotzdem ersteres schwarz und letzteres weiß aussieht. Erst der Kontext bedingt, daß nicht nur die Intensitätsverhältnisse zwischen den einzelnen Wellenlängenbereichen des von einem einzelnen Objekt reflektierten Lichtes, sondern auch die zwischen den von verschiedenen Gegenständen ausgestrahlten Lichtwellen bestimmt werden können, so daß ein Betrachter in die Lage kommt, weiße von schwarzen Flächen unterscheiden zu können. Vergleichbares gilt für die Farbpaare Orange und Braun sowie Grüngelb und Olivgrün (und wahrscheinlich noch für einige weitere Brauntöne).

Beide Formen von Simultankontrast erlauben dem Physikalisten nicht, Farben als Eigenschaften von einzelnen Gegenständen anzusehen. Das dies ein ernsthaftes Problem darstellt, geben einige Objektivisten ohne weiteres $\mathrm{zu}$. Armstrong versucht deshalb mehr oder weniger überzeugend, einen Ausweg aus diesen Schwierigkeiten zu finden, indem er zum einen auf einen möglichen, objektiven Unterschied zwischen den Farben der einzelnen Farbpaare hinweist, und um anderen sich die Option offenhält, bei dem Simultankontrast ebenfalls von einer Fehlrepräsentation zu sprechen (vgl. Armstrong (1993): 273). Letzteres ist jedoch nicht so einfach aufrechtzuerhalten, da im Grunde das Phänomen des Simultankontrastes einen wesentlichen Bestandteil unserer Farberkennung verkörpert. So kommt es auch, daß viele Philosophen oder Wissenschaftler lieber die Alternative des Subjektivismus akzeptieren, als hier von Farbillusionen zu sprechen (wie etwa Hardin (1988), aber auch Zeki (1993) es bevorzugen). Denn auch wenn Armstrong zwar zurecht bemerkt, daß dieser Kontrast von uns nur sehr selten bemerkt wird, heißt das noch nicht, daß er auch nur sehr selten vorliegt: tatsächlich legt die Neurophysiologie nahe, daß jede Farbwahrnehmung auf dem Simultankontrast beruht (vgl. Zeki (1993): Kap. 23; vor allem: 231f.). Und außerdem wird der Simultankontrast nicht auf kausale Interaktionen zwischen den wahrgenommenen Flächen zurückzuführen sein, sondern vielmehr durch kognitive Prozeße erklärt werden müssen.

Wie sieht es dann mit der Alternative des objektiven Unterschiedes zwischen den betreffenden Farben aus? Hinsichtlich des Intensitätskontrastes findet sich, wie gesagt, ein physikalisch beschreibbarer und von uns wahrnehmbarer Unterschied in dem proportionalen Wert, wieviel Licht jeweils 
reflektiert wird: weiße Gegenstände senden mehr Licht zurück als schwarze. Der Intensitätskontrast bereitet also nicht wirklich Probleme. Aber im Falle des Farbkontrastes sieht dies anders aus: es wird einen solchen, objektiven Unterschied nicht geben, da der intrinsisch realisierte Reflektanztyp der in verschiedenen Kontexten als unterschiedlich gefärbt gesehenen Fläche dabei unverändert bleiben kann. Es kann sich also nur um eine Farbillusion handeln, wenn man Farben mit den physikimmanenten oder dispositionalen Reflektanzeigenschaften der Gegenstände identifiziert: eine Konsequenz, die der Physikalist aus dem eben gegebenen Grund besser vermeiden sollte. Aber es scheint doch noch einen Ausweg zu geben: und zwar Farben nicht als intrinsische oder dispositionale Eigenschaften einzelner Objekte, sondern als interne Relationen zwischen den betrachteten Objekten anzusehen. Damit sind Farben nicht mehr mit den Reflektanzprofilen oder -typen gleichzusetzen, sondern mit einer von diesen realisierten Art von internen Relationen.

Der Physikalismus wird dabei aufrechterhalten, daß Farben immer noch auf den intrinsischen Beschaffenheiten der Farbträger (und eventuell den bestehenden Naturgesetzmäßigkeiten) supervenieren und somit der repräsentationale Aspekt der Intuitiven Farbkonzeption nicht verletzt wird: auch wenn Farben streng genommen nicht mehr nur Eigenschaften der einzelnen Gegenstände für sich genommen sind. Danach nehmen wir mithilfe unseres visuellen Sinnes bestimmte Ähnlichkeiten und Verschiedenheiten zwischen Gegenständen wahr. Man könnte gegen diesen Vorschlag einwenden, daß er nicht mehr das Phänomen der Farbkonstanz erklären könnte, da ein Gegenstand mit jeder Veränderung des Kontextes doch auch seine Farbigkeit wechseln würde: was nun eben offensichtlich meistens nicht der Fall ist. Dieses Problem ergibt sich aber nicht wirklich, weil der Simultankontrast in den meisten Fällen so schwach ist, daß er von uns einfach nicht mehr bemerkt wirkt. Wenn die Farbe eines Gegenstandes in zwei Kontexten leicht verschieden ist, und wenn zudem unsere Wahrnehmungen von ihm in den zwei zeitliche getrennten Fällen sich phänomenal leicht unterscheiden, müssen wir dies jedoch nicht immer unbedingt bemerken, weil wir den Gegenstand nicht in beiden Situationen gleichzeitig sehen. Erst, wenn die Möglichkeit des Vergleiches besteht, wird die Differenz auffallen. Es besteht also die berechtigte Hoffnung, die Schwierigkeiten, die der Farbtonkontrast mit sich bringt, in den Griff zu bekommen, auch wenn hier 
dieser Weg nicht genauer erkundet werden soll. Ganz unabhängig davon ist der Intensitätskontrast auf jeden Fall kein Problem für den Farbphysikalismus.

\subsubsection{Mit der Intuitiven Farbkonzeption verbundene Einwän- de}

In diesem Abschnitt geht es nun um Einwände, die die Natur der Farben einerseits und ihre introspektive Zugänglichkeit andererseits betreffen. Ein erster Einwand gegen einen Farbphysikalismus kann auf der Basis vorgebracht werden, daß uns viele Aspekte der objektiven Natur von Farben nicht zugänglich sind. Insbesondere sind uns die Farbeigenschaften weder als Reflektanzprofile, noch als Reflektanztypen gegeben: wir sehen Farben nicht als physikalisch beschreibbare Eigenschaften. Warum sollten sie dann als solche angenommen werden? Eine negative Antwort auf diese Frage ist durch die Offensichtlichkeitsthese motiviert, welcher zufolge uns die Natur der Farben ganz genau so in unseren Farbwahrnehmungen erscheint und introspektiv gegeben ist, wie sie auch tatsächlich ist. Doch die Akzeptanz dieser These setzt bereits die Ablehnung des Objektivismus voraus. Denn wenn Farben eine objektive Natur haben, dann ist es weder erforderlich, daß diese uns auch wirklich in unseren Sinneserfahrungen gegeben ist, noch, daß wir überhaupt auf alle Aspekte ihrer Natur solchen Zugriff haben können. Der Physikalist wird keineswegs zugeben, daß uns alle Wesensmerkmale der Farben sinnlich oder introspektiv zugänglich sind. Somit lehnt er die Offensichtlichkeitsthese (OS) ab und optiert stattdessen für die schwächere Transparenzthese (TT), oder beschränkt sich sogar auf die noch schwächere These (REP). Auch die Unbestimmtheit der Wahrnehmung, das heißt, das eingeschränkte Aufnahmevermögen des visuellen Sinnesorganes spielt hierfür eine Rolle.

„Perception does not reveal the whole truth about colors and the truth it does reveal is delimited by the characteristics of our perceptual systems. [...] Once again, we need to be careful in our analysis of the relation between perception and reality in order to avoid the fallacy of total information." (Hilbert (1987): 27; 107) 
Der Objektivismus bedingt geradezu die Ansicht, daß nicht alle Wahrheiten über die Farben uns in Farbwahrnehmungen gegeben sein können. Diese Forderung an ihn zu stellen, ist also nicht mehr als question-begging. Ein Subjektivismus kann nicht einfach voraussetzen, daß die Natur der Farben bereits von der Intuitiven Farbkonzeption erschöpft wird, will er sinnvoll gegen einen Objektivismus argumentieren. Um die mit den Farben identischen Reflektanzeigenschaften repräsentieren zu können, müssen wir diese also nicht unbedingt als Reflektanzen (oder gar als die diesen zugrundeliegenden, mikrophysikalischen Eigenschaften) wahrnehmen. Farben müssen uns nicht einmal ganz allgemein als physikalische Eigenschaften gegeben sein: es genügt, wenn wir sie als Merkmale makroskopischer Gegenstände sehen (die beiden Subjektivisten Boghossian \& Velleman kommen zu demselben Schluß in (1991): 72ff.). Doch Johnston schließt hieran ein weiteres Argument an. Da die physikalischen Reflektanzeigenschaften nicht sichtbar sind, sind es auch nicht die Farben: aber dies ist offensichtlich nicht das, was Physikalisten gerne haben möchten, weil sie Farben ja als wahrnehmbare Eigenschaften einstufen. Seine Begründung für den Schluß ist ganz einfach:

„A particular counts as visible only if it has visible properties and it has visible properties only if it has properties with whose natures vision acquaints us. That is to say that although it is a necessary condition of a property $\mathrm{F}$ being visible that something's having $\mathrm{F}$ at some time explains a visual experience, this is not sufficient. For many fundamental physical properties satisfy this necessary condition while not being visible properties. They fail to count as visible properties because vision does not acquaint us with the nature of these properties but only with their effects. [...] Obviously acquaintance can be a matter of degree on this view. So we do not need a complete revelation of the nature of a property to be acquainted with the property.“ (Johnston (1992): 164)

Dieser Einwand entspricht in vielem der klassischen, indirekt-realistischen Idee, daß nur sekundäre Qualitäten uns direkt gegeben sind, während wir nur aufgrund dieser auf primäre schließen können. Auch die Aktualismusthese der Sinnesdatentheoretiker hält hier versteckt wieder Einzug (nicht von ungefährt beruft Johnston sich auf Russell und vertritt stellt eine an Jacksons Vorschlag (1977) orientierte, repräsentative Wahrnehmungstheo- 
rie: vgl. Johnston (1992): 172ff.)). Johnston unterstützt sein Argument nun noch durch ein einfaches Beispiel. Es ist vorstellbar, daß ein bestimmtes Reflektanzprofil - entweder als solches, oder weil es von einem bestimmten Typus ist - in einem Subjekt unter Normalbedingungen immer sowohl eine eindeutig festgelegte Farb- als auch eine Klangwahrnehmung hervorruft, so daß mit gutem Recht gesagt werden kann, daß beide Sinneserfahrungen ein- und dieselbe Reflektanzeigenschaft in der Welt repräsentieren. Eine Möglichkeit, diesen Fall zu realisieren, bietet vielleicht die Synästhesie; eine andere läßt sich für Lebewesen finden, die nicht nur über Augen, sondern auch über ein einem Echolot ähnelndes Sinnesorgan verfügen, welches jedoch zu Klangqualia führt (vgl. Johnston (1992): Fn. 33). Ein Notwendigkeitsobjektivist wird solche Fälle sicherlich leugnen. Aber auch davon abgesehen bleibt dem Objektivisten eine Erwiderung.

Zuerst einmal ist es nicht gesagt, ob das Argument auch den Schwachen Physikalisten trifft, da dieser Farben nicht als physik-immanente Eigenschaften ansieht. Es ist ebenso ungeklärt, ob nicht auch die Natur makroskopischer, physikalischer Eigenschaften sinnlich erfaßt werden kann. Doch in jedem Fall ist das Sichtbarkeitskriterium vor dem Hintergrund einer direkt-realistischen und externalistischen Wahrnehmungstheorie nicht haltbar. Es bleibt auch dann die Bedingung erhalten, daß Gegenstände nur vermittels ihrer Eigenschaften gesehen werden können. Aber die Natur der Eigenschaften muß uns nicht gegeben sein: es genügt allein die passende, nomologische Korrelation und, vielleicht, die Möglichkeit, Gehaltsunterschiede - bis zu einem gewissen Grade - introspektiv feststellen zu können. Also auch wenn das Beispiel von Johnston möglich ist, handelt es sich um einen Fall, in dem zwei verschiedene, sinnliche Wahrnehmungen vorliegen - eine visuelle und eine auditive -, die zufälligerweise dieselbe Eigenschaft in der Welt repräsentieren. Die externalistisch erklärte Wahrnehmbarkeit kann und wird Johnston eventuell auch gar nicht angreifen, da sie bereits durch die nomologische Korrelationen gegeben ist. Was ihm vielleicht viel wichtiger ist, ist der Umstand, daß uns die Reflektanzeigenschaft auf zwei verschiedene Weisen phänomenal gegeben ist. Dies stellt für sich genommen jedoch noch kein Problem für den Physikalisten dar, da uns zum Beispiel auch Formeigenschaften in verschiedenen Modalitäten mit unterschiedlichen Phänomenalitäten gegeben sind - es sei denn, die 
Phänomenalität der Farben wird als wesentlicher Bestandteil ihrer Natur angesehen, wie es Johnston auch tut. Doch hierbei handelt es sich bereits um ein ganz anderes Argument.

Bevor diese Problem jedoch wieder aufgegriffen wird, soll noch kurz auf die beiden Merkmale der Einheitlichkeit und der Einfachheit eingegangen werden. Die Disjunktivität des Schwachen Physikalismus scheint der Einheitlichkeit $\mathrm{zu}$ widersprechen. Doch dies stimmt nur bedingt, da es nicht klar ist, was die Einheitlichkeit überhaupt aussagt. Wenn sie nicht mehr fordert, als daß jeder Farbe ein ihr allein zukommendes Merkmal und den Farben insgesamt ein gemeinsames Charakteristikum zuzusprechen ist, dann wird jede Theorie der Farben diese Bedingung erfüllen, da es ja gerade ihr Ziel sein sollte, entweder die Klasse der Farben und deren einzelne Mitglieder zu analysieren, oder aber diese gleich als einheitliche, primitive Eigenschaften anzusehen. Wenn die Forderung dagegen darin besteht, ein einheitliches, intrinsisches Merkmal für die Farbeigenschaften zu fordern, dann wird der Schwache Physikalismus die Einheitlichkeit nicht mehr gewährleisten können. Nun ist die Disjunktivität für sich genommen zwar ontologisch nicht ganz unproblematisch; aber es ist nicht einsichtig, warum Farben ihre Einheitlichkeit nicht auch über ihre Dispositionalität erhalten können sollten. Denn würde es einen offensichtlichen, phänomenalen Unterschied ausmachen, ob intrinsische oder dispositionale Eigenschaften von uns wahrgenommen werden, dann stellte sich wahrscheinlich gar nicht die Alternative zwischen dem Starken und dem Schwachen Physikalismus, da einer der beiden sofort als die angemessenere Theorie ersichtlich wäre.

Solange also nicht eine phänomenale Differenz hinsichtlich einer durch Physik-Immanenz und einer durch Subjekt-Realivität bewirkten Einheitlichkeit festgestellt oder sinnvollerweise postuliert werden kann, ist es plausibel, das Merkmal der Einheitlichkeit als erfüllt - oder wenigstens nicht als nicht-erfüllt, sondern als neutral - anzusehen (vgl. Jackson (1996): 212ff.). Und selbst im Ernstfall sollte an dem einheitlichen Charakter der Farbeigenschaften nicht allzuviel hängen (vgl. Maund (1982): 312f.). Jackson führt als Bekräftigung hierfür das Beispiel von unserem Begriff von Jade an, der, wie sich im Nachhinein herausgestellt hat, zwei sich zwar ähnelnde, aber doch verschiedene Gesteinsarten bezeichnet (vgl. Jackson (1996): 216). Etwas anders sieht dies mit Hinsicht auf die relative Einfachheit der Farben aus. Die physikalisch beschreibbare Natur der Far- 
ben ist sicherlich alles andere als einfach. Man kann sich zwar fragen, inwieweit Farben überhaupt als einfache Eigenschaften gesehen werden (ob nicht zum Beispiel die drei voneinander unabhängigen Parameter Farbton, Helligkeit und Sättigung sowie die vielschichtigen, phänomenalen Beziehungen bereits eine Art von Komplexität bedeuten). Aber die Physikalität bringt es auf alle Fälle mit sich, daß Farben längst nicht so einfache Merkmale sind, wie sie uns zu sein scheinen. Hierin liegt gerade eine der Motivationen dafür, einen Farbprimitivismus zu vertreten: denn primitive Eigenschaften zeichnen sich gerade durch die ihre Einfachheit und Unanalysierbarkeit aus.

Doch das Hauptargument gegen den Farbphysikalismus besteht in dem Vorwurf, dem phänomenalen Aspekt der Intuitiven Farbkonzeption nicht gerecht werden zu können: das heißt, den phänomenalen Charakteren, Merkmalen und Beziehungen, die den Farben eigen zu sein scheinen. Der Physikalismus soll entsprechend die Phänomenalität der Farben weder aufrechterhalten, noch erklären können. Wenn dies zutrifft, kann der Farbphysikalismus demgemäß - neben der Einfachheit - auch nicht den Hauptbestandteil der These (PHE) beibehalten und muß folglich die Transparenzthese (TT) aufgeben, die ja Farben als phänomenale und als repräsentationale Eigenschaften ansieht. Hinter diesem Argument verbirgt sich die Idee, daß die Merkmale des phänomenalen Aspektes des introspektiven Charakters von Farbwahrnehmungen eigentlich zur Natur der Farben dazuzurechnen sind, aber im Grunde nur den Farbqualia zukommen können. Introspektion ermöglicht es uns dabei, von der den Farben wesentlichen Phänomenalität Kenntnis zu erhalten. Die These (PHE) soll dann garantieren, daß eine Theorie der Farben unserer intuitiven, gewöhnlichen Auffassung von denselben auch in diesem Aspekt gerecht wird. Die Überzeugung, daß nicht nur die Farbqualia, sondern auch die Farben eine Phänomenalität aufweisen müssen, findet sich wahrscheinlich bei einer Mehrzahl der Philosophen, die sich mit Farben beschäftigen: und nicht nur bei Subjektivisten, sondern vor allem auch bei den objektivistischen Vertretern der These (REP), die einen Objektivismus ${ }_{\mathrm{NEC}}$ befürworten. ${ }^{27}$ Eine gute und knappe

$27 \mathrm{Zu}$ den Subjektivisten, die diese These vertreten, zählen: Boghossian und Velleman ((1991): 85f.) (vgl. auch ihr Zitat im Abschnitt über die Intuitive Farbkonzeption); Hardin ((1986): 66); Thompson ((1995): 122ff.); Johnston ((1992): 149ff.; 164f.). Unter den Objektivisten akzeptieren oder untertützen unter ande- 
Formulierung der Kritik findet sich bei Keith Campbell:

„These aspects of colour vision - that there are just the four unique hues, that there are just the mixtures and resemblances that there are, and that every colour has exactly one complementary - are so intimately a part of the nature of colours as we experience them that one is tempted to say they belong to the essence of the colours. Whether or not that is a fortunate way to express the matter, any reductive realism that involves identifying colours with physical features of surfaces or light waves must omit these aspects or relegate them to some accidental, marginal status. They must appear as the most contigent of side issues, since they have no basis in the external physics of the situation. Such a dismissive stance is unacceptable.“" (K. Campbell (1993): 264)

Die Liste der nicht-erklärbaren Merkmale von Farben läßt sich noch erweitern: neben Reinheit und Gemischtheit, Ähnlichkeiten und Verschiedenheiten sowie Komplementarität gibt es noch die aus Farbton, Helligkeit und Sättigung bestehende Dreidimensionalität, die Gemeinsamkeiten und Unterschiede von chromatischen und achromatischen Farben, der qualitative Charakter von Farben an sich, die Inkompatibilitäten (daß es zum Beispiel kein rötliches Grün gibt) sowie der Umstand, daß jede chromatische Farbe höchstens aus zwei reinen Farben zusammengesetzt ist (die Binarität). Worin liegt nun aber genau das Problem für den Physikalisten mit dieser Farbphänomenalität? Warum kann er (PHE) nicht aufrechterhalten? Das Problem für die Objektivisten ist, daß sie Farben ontologisch strikt von Farbqualia (als Eigenschaften von mentalen Zuständen) trennen: es gibt keine ontologische Beziehungen zwischen den Farbeigenschaften und den entsprechenden Farbwahrnehmungen. Das heißt, die introspektierbaren Merkmale der Qualia können nicht so ohne weiteres auf die Farben übertragen werden. Die Objektivisten müssen also einen anderen Weg finden, um zu gewährleisten, daß die Phänomenalität den Farben auf die eine oder andere Weise zugesprochen werden kann.

Dafür muß zuerst einmal die Notwendigkeitsthese $\left(\mathrm{NEC}_{\mathrm{NB}}\right)$ angenommen werden (vgl. Byrne \& Hilbert (1997c): 267). Eine kontingente Verbin-

rem die folgenden die Kritik: Hilbert ((1987): 115ff.); Byrne \& Hilbert ((1997c): 274ff.); Tolliver ((1994): 416f.). Bei Keith Campbell ist es unklar, welche Position er genau vertritt; aber er scheint einen schwachen Objektivismus zu bevorzugen (vgl. K. Campbell (1993): 265). 
dung erlaubt die Möglichkeit, daß ein- und dieselbe repräsentierte Farbeigenschaft selbst unter den den Umständen entsprechenden Normalbedingungen mit verschiedenen Phänomenalitäten gesehen werden kann (und umgekehrt): wie es etwa in den Beispielen von Qualia-Inversion beschrieben wird. Damit kann aber die Phänomenalität kein wesentlicher Bestandteil der Natur der Farben sein. Hierfür ist zumindest eine notwendige Korrelation von repräsentierter Farbe und Farbquale vonnöten. Doch von sich aus gewährleistet eine direkt-realistische und externalistische Gehaltstheorie nur eine kontingente Beziehung, da die relevanten nomologischen Korrelationen - wie die Naturgesetzmäßigkeiten auch - von Welt zu Welt differieren können (vgl. die Diskussion der externalistischen Gehaltstheorien in Boghossian \& Velleman (1991): 86ff.).

Dieser Einwand wird dementsprechend von den Physikalisten auch als zutreffend akzeptiert. Diese haben nun grundsätzlich zwei Möglichkeiten, dem Problem für ihre Position zu entgehen und somit auf die Kritik zu antworten: entweder finden sie - mithilfe der Notwendigkeitsthese - eine Möglichkeit, die fraglichen Merkmale durch Charakteristika der physikalischen Farbeigenschaften zu erklären; oder sie leugnen ganz einfach die

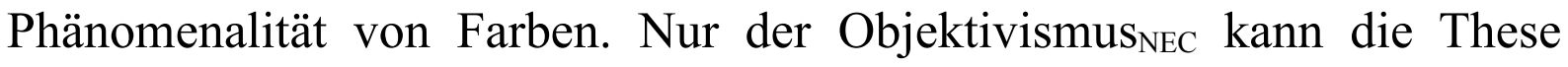
(PHE) für den Physikalisten retten; aber der Objektivismus ${ }_{\mathrm{ACT}}$ ist vielleicht doch nicht so unplausibel, wie es scheinen mag. Denn es ist wirklich nicht so klar, ob die Meinung, daß Farben nur kontingenterweise von uns als phänomenal gesehen werden, auf jeden Fall abgelehnt werden sollte. Das folgende Zitat von Armstrong läßt sich so interpretieren, daß er einer der Objektivisten ist, die zu der Akzeptanz dieser Überzeugung tendieren und somit die Phänomenalität nicht der Natur der Farben zurechnen.

„The phenomenology of colours appears to tell us this: these properties are simple or relatively simple; and they are completely distinct from the primary properties of things in the environment, or possibly in the brain, with which the colours are correlated. [...] While feeling the force of the phenomenological case, I wish to resist it. [...]

If there is anything that we seem to have a perceptual grip on, it is the internal nature of colours. How can this be explained? Here I suggest that the very complex relations of resemblance and difference between the whole array of the colours, with its three 'dimensions' of hue, saturation and intensity, relations which are so vividly presented in visual perception, creates in us the impression that we 
are given in perception the qualities from which these resemblances flow. After all, it is common enough for what are really relational entities to present themselves as something non-relational. Up and down is a relative notion, but it presents itself to us as absolute.

If all this is correct, then the determinables and determinates that colour-perception presents us with will be much, much, messier affairs than those to be found in the agreeable cases of length, duration and mass. But they need not be any different in principle, except that the properties involved are very unlikely to be universals, and instead are 'second-class' properties." (Armstrong (1997): 58f.; 60f.)

Die Idee Armstrongs im Umgang mit den phänomenalen Beziehungen scheint die folgende zu sein: es ist nicht nur möglich, daß ein Teil der Natur der Farben von uns nicht sinnlich erfaßt werden kann, sondern auch, daß Farben uns anders präsentiert werden, als sie eigentlich sind. Wenn relationale Eigenschaften als nicht-relational wahrgenommen werden können, warum sollten physikalische Farben dann nicht auch als nicht-physikalisch, und nicht-phänomenale Farben als phänomenal gesehen werden? Der erste Schritt weist noch einmal auf das eben im Zusammenhang mit der Offensichtlichkeitsthese vorgebrachte Argument der Subjektivisten hin: demnach ist es kein Problem, physikalische Eigenschaften wahrzunehmen, ohne sie als physikalische zu erfahren. Schwieriger wird es dagegen mit dem umgekehrten Fall: kommt den Farben die Phänomenalität nur illusionär zu, wenn man annimmt, daß die introspektiv zugänglichen, phänomenalen Beziehungen wenigstens scheinbar die Natur der Farben notwendigerweise mitbestimmen? Die Analogie der internen Relation zwischen Oben und Unten hilft da nicht weiter. Es ist zwar einsichtig, daß wir interne Relationen nicht unbedingt als interne wahrnehmen, aber das macht es nicht plausibel, auch anzunehmen, daß wir manchmal interne Ähnlichkeitsrelationen oder andere Merkmale dort sehen, wo es sie in Wirklichkeit gar nicht gibt.

Trotzdem scheint die These Armstrongs zu sein, daß die Phänomenalität den Farben nur kontingent zuzusprechen ist: das Vorhandensein der phänomenalen Merkmale und Beziehungen bei Farbwahrnehmungen erweckt in uns zwar den Eindruck (the impression), als ob wir wirklich phänomenale Eigenschaften wahrnehmen würden, aber dieser Eindruck täuscht. Nun läßt sich die zitierte Stelle vielleicht auch auf eine andere 
Weise interpretieren: daß Armstrong immer noch hofft, daß eine Objektivierung der Phänomenalität möglich ist und deswegen Farben als primäre Qualitäten anzusehen ist. Der eben erwähnte, introspektiv erfahrene Eindruck wäre demnach also berechtigt. Auch wenn die obigen Ausführungen eher für die Leugnung einer phänomenalen Natur von Farben sprechen, gibt es in einigen älteren Texte Hinweise darauf, daß Armstrong doch lieber den Weg gehen möchte, sowohl die Objektivität als auch die Phänomenalität beizubehalten. ${ }^{28}$ Da eine wirklich klare Aussage von ihm hierzu fehlt, kann es letztlich nur bei Spekulationen über seine Ansicht bleiben. Es ist nur sicher, daß Armstrong auf alle Fälle viel eher die Phänomenalität als die Objektivität aufzugeben bereit ist. Ob dies von einem Objektivismus ${ }_{\mathrm{NEC}}$ vermieden werden kann, soll weiter unten geklärt werden. Bis dahin ist es angebracht, auf die Konsequenzen hinzuweisen,

28 Vergleiche dazu:

„Colour could well be a very unimportant and extremely idiosyncratic physical property. The perceived relationships between the colours could be very unimportant, and idiosyncratic, relations between these properties. (These relationships might nevertheless be necessary because flowing internally from the nature of the unimportant and idiosyncratic properties)." (Armstrong (1993): 272)

Die physikalischen Äquivalente von Farben könnten somit möglicherweise doch selbst die phänomenalen Beziehungen aufweisen oder erklären. Diese Hoffnung wird aber in Frage gestellt durch den folgenden Beleg, demzufolge die Phänomenalität doch nur eine Illusion sein könnte:

„But I suggest that it is the fact that we grasp these resemblances among the secondary qualities so clearly and comprehensively that generates, or helps to generate, the illusion that we have grasped in a concrete way the nature of the resembling things. In an unselfconscious way, we are all perfectly aware that resemblances are [as internal relations - der Autor] completely determined by the natures of the resembling things. So given resemblances, we automatically infer natures. We know further that in perception what is automatically inferred is regularly felt as directly given, as contrasted with being inferred. (Consider for example the way we pass from hearing words to the semantic intentions of speakers.) So in perception of the secondary qualities, we have a vidid impression of intrinsic nature." (Armstrong (1987): 13) 
die ein Objektivismus ${ }_{A C T}$ mit sich bringt, der Farben jegliche phänomenale Merkmale abspricht (da alle Merkmale des phänomenalen Aspektes mehr oder weniger mit dem qualitativen Charakter für sich genommen - dem Farbquale - zusammenhängen, ist es kaum zu erwarten, daß nur ein Teil der Merkmale zurückgewiesen wird).

Wenn es keine phänomenalen Farben geben soll, dann muß die introspektierte Phänomenalität anders erklärt werden. Der Objektivist wird dabei den einfachen Weg gehen und letztere den Farbqualia zuschreiben. Geht man allein von der These $\left(\mathrm{ACT}_{\mathrm{NB}}\right)$ aus, dann sind beispielsweise Fälle von Qualia-Inversion erlaubt (und Armstrong sowie Smart sind diesen nicht abgeneigt gegenüber (vgl. Armstrong (1993): 272); Smart (1975): 56): ein- und dieselbe physikalische Eigenschaft kann unter den jeweiligen Normalbedingungen in unterschiedlichen Welten zu verschiedenen Farbempfindungen führen. Die Farbe wird dabei über die faktischen, normalen Wahrnehmungen von Menschen in der aktualen Welt individuiert, während ihre Natur vollständig physikalisch beschreibbar ist und somit in allen Welten dieselbe bleibt, auch wenn sie verschiedenartige Sinneserfahrungen hervorruft. Welches Quale die Wahrnehmungen einer bestimmten Farbe in den jeweiligen Welten haben, ist somit kontingent. Damit wird natürlich die These (PHE) - und somit auch die Transparenzthese (TT) - aufgegeben, derzufolge Farben eine phänomenale Natur besitzen. ${ }^{29}$ Eine Aufspaltung der Intuitiven Farbkonzeption ist die Folge: während die Merkmale des repräsentationalen Aspektes den repräsentierten Farbeigenschaften zukommen, zeigen die Farbqualia die Merkmale des phänomenalen Aspektes.

Eine solche Position steht jedoch einem weiteren Problem gegenüber (vgl. Armstrong (1993): 272; (1997): 60). Die physikalisch beschreibbare Natur der Farben ist in unseren Farbwahrnehmungen offensichtlich nicht gegeben. Die uns introspektiv zugängliche phänomenale Natur kommt nur den Qualia, nicht aber den repräsentierten Farben selbst zu. Das hieße doch

29 Es ist nicht klar, inwiefern dies Armstrongs anderen Auffassungen widerspricht. Er hat selbst zum Beispiel eine Variante der Transparenzthese vertreten, aber dies ist ihm nur dann möglich, wenn er eine repräsentationalistische, das heißt, rein externalistische Theorie der Qualia sowie die Notwendigkeitsthese $\left(\mathrm{NEC}_{\mathrm{NB}}\right)$ akzeptiert: andernfalls kann er nicht sagen, daß die Phänomenalität in dem einen oder anderen Sinne auf die repräsentierten Eigenschaften in der Außenwelt zutrifft. 
aber, daß uns einerseits die Natur der Farben nicht zugänglich ist, während uns die Natur von anderen Eigenschaften in den fraglichen Sinneserfahrungen offenbart wird: wie könnte es sich dann dabei noch um Wahrnehmungen von physikalischen Eigenschaften (ob als Farben verstanden oder nicht) handeln $?^{30}$ Ganz so einfach ist es nun jedoch nicht, denn uns ist die Natur der objektiven Farben wenigstens zu einem Teil erschlossen: aufgrund unserer Farbwahrnehmungen erkennen wir ihre Externalität, Intrinsität, Objektivität, Repräsentierbarkeit und ihre Erklärungskraft. Nur um die konkreten Unterschiede zwischen einzelnen, repräsentierten Farben zu erfassen, müssen wir uns der Qualia bedienen. Dabei ist es ausreichend, wenn jedem Qualiaunterschied auch eine Gehaltsdifferenz entspricht, und umgekehrt. Es ist weder zusätzlich gefordert, daß die beiden Unterschiede von ein- und derselben Eigenschaftsart gelten müssen, noch, daß unter allen Bedingungen ein bestimmtes Quale immer mit derselben Farbe korreliert sein muß. Und schließlich ist es nicht gesagt, daß etwas Nicht-Wahrgenommes auch tatsächlich nicht da ist:

„The perceived relationships between colours could be very unimportant, and idiosyncratic, relations between these properties. [...] These unimportant properties seem so prominent in colour perception, [for] what it not perceived seems not to be there, and what is perceived fills the phenomenological stage and so the mental stage." (Armstrong (1993): 272)

„The headless woman illusion is certainly of importance here... What it shows is the naturalness of an illegitimate transition from a failure to perceive something (that the woman has a head) to a 'perception' of something unreal (the woman has no head). The illusion is rather easily brought about by presenting the woman against an illuminated black background with a black cloth over her head. [...] Our failure to perceive the identity of the colours with certain primary properties, and our failure to grasp their full complexity, could actually generate the illusion that we could observe that they are not identical with any primary properties and that they are objectively relatively simple." (Armstrong (1997): 60)

Dies alles wird durch die auf Normalbedingungen beschränkte Aktualitätsthese $\left(\mathrm{ACT}_{\mathrm{NB}}\right)$ des Objektivisten erfaßt. Und der Objektivismus ist ohne

30 Im Grunde handelt es sich hier um eine Variante des oben bereits erwähnten Sichtbarkeitsargumentes von Johnston. Boghossian und Velleman argumentieren auf sehr ähnliche Weise gegen den Physikalismus (vgl. (1991): 98ff.). 
weiteres damit konsistent, daß die physikalische Natur der Farben nicht unmittelbar wahrgenommen werden kann, solange es uns visuell-sinnlich möglich ist, Farbunterschiede zu sehen: wobei die Qualia als eine Art von eng-funktional bestimmten, phänomenalen Gegebenheitsweisen hinsichtlich der Repräsentation der Farben fungieren können (vgl. dazu mehr im Schlußkapitel). Zusammenfassend läßt sich also sagen, daß ein Objektivismus $_{\mathrm{ACT}}$ - zusätzlich zu den im Vergleich zwischen den beiden physikalistischen Alternativen erwähnten Schwierigkeiten - im wesentlichen den Nachteil zeigt, daß er die Phänomenalität von Farben nicht erklären kann und somit sowohl die These (PHE) als auch die Transparenzthese (TT) ablehnen muß. Es wird sich zeigen müssen, ob andere Theorien der Farben zu einer besseren Bewertung gelangen werden können.

\subsection{Argumente gegen den Notwendigkeitsobjektivis- mus}

Der Objektvismus $_{\mathrm{NEC}}$ begründet sich von der Transparenzthese (TT) her, deren Wahrheitsanspruch gerecht zu werden, als ein erstrebenswertes Ziel für jeden Farbtheoretiker gelten kann. Wenn Farben ihrer Natur nach sowohl repräsentierte als auch phänomenale Eigenschaften sind, die Phänomenalität der Farben aber in erster Linie den Farbqualia zukommt, muß eine besonders enge Beziehung zwischen Farben und Farbqualia bestehen. Der Repräsentationalismus bietet hierfür zwei alternative Lösungen an: entweder können die Farbqualia direkt mit den repräsentierten, objektiv und extern instantiierten Farbeigenschaften identifiziert werden (vgl. Dretske (1995a); (1995b)); oder aber das Quale einer Wahrnehmung ist vollkommen durch deren Gehalt bestimmt, daß heißt, durch dieselben, externen Faktoren, die die repräsentierte Eigenschaft spezifizieren (vgl. Tye (1995): 137ff.). ${ }^{31}$ Für eine Theorie der Farben ist es jedoch unwichtig, auf diesen Unterschied genauer einzugehen. Dagegen sind einige mit der repräsentationalistischen Position verbundene Konsequenzen von Bedeu-

31 Der Repräsentationalismus erfordert sogar einen Farbobjektivismus, geht man von einer externalistischen Gehaltstheorie aus. Dies wird dadurch leicht einsichtig, da $\beta$ er die Transparenzthese (TT) akzeptiert, die unter anderem Farben als objektive Eigenschaften ansieht (vgl. Tye (1995): 30f.; 144ff.). 
tung. Eine erste ist die bereits angesprochene Akzeptanz der Notwendigkeitsthese: es muß eine notwendige Verbindung zwischen Gehalt und Quale bestehen, da sonst beide nicht identisch oder immer durch dieselben Faktoren bestimmt sein können. Wiederum maßgebend für die Anwendung dieser Idee auf eine Theorie der Farben sind Byrne und Hilbert, auf deren Aufsatz sich mittlerweile Repräsentationalisten wie Tye oder Dretske beziehen, sobald es um Farben geht.

„Our physicalist theory of color has two main components. First, colors are types of surface spectral reflectances. Second, color content and color phenomenology necessarily go together.“ (Byrne \& Hilbert (1997c): 264)

Die Frage ist nun, wie der Objektivismus ${ }_{\mathrm{NEC}}$ die These (PHE) für Farben aufrechterhalten kann. Dabei lassen sich generell zwei Gründe für eventuelle Schwierigkeiten ausmachen: zum einen kann vielleicht auch der Objektivismus $_{\mathrm{NEC}}$ letztlich die Phänomenalität der Farben nicht erklären; zum anderen kann die Notwendigkeitsthese $\left(\mathrm{NEC}_{\mathrm{NB}}\right)$ möglicherweise nicht vertreten werden. Beide Probleme sollen nun im folgenden nacheinander besprochen werden, wobei mit der Begutachtung der Plausibilität der Notwendigkeitsthese begonnen wird.

\subsubsection{Argumente gegen die Notwendigkeitsthese an sich}

Zuerst einmal soll kurz daraufhingewiesen werden, daß der Starke Physikalismus die Notwendigkeitsthese nicht so ohne weiteres wird annehmen können, da er schon nicht die mit der ersteren eng zusammenhängenden Aktualitätsthese in ihrer ursprünglichen Form akzeptiert. Die beiden Thesen unterscheiden sich nur in dem Grad ihrer metaphysischen Notwendigkeit. Darüberhinaus impliziert die These $\left(\mathrm{NEC}_{\mathrm{NB}}\right)$ ihr aktuales Gegenstück $\left(\mathrm{ACT}_{\mathrm{NB}}\right)$ - (gleiches gilt natürlich auch für die allgemeinen, von den Normalbedingungen unabhängigen Varianten (NEC) und (ACT), die aber im folgenden vernachlässigt werden sollen). Wenn nun der Starke Physikalist Farbgehalte feingradiger als die entsprechenden Farbqualia individuiert, kann er $\left(\mathrm{ACT}_{\mathrm{NB}}\right)$ nicht mehr in der ursprünglichen Form aufrechterhalten. Dasselbe gilt damit auch für $\left(\mathrm{NEC}_{\mathrm{NB}}\right)$, welche sich somit nicht mehr auf die 
maximal spezifizierbaren, sondern nur noch auf die ebenso feingradig wie Qualia individuierten Gehalte beziehen kann. Dieser Umstand verhindert zwar nicht die Möglichkeit eines Starken Physikalismus, vermindert aber dessen Attraktivität für einen Notwendigkeitsobjektivisten. Diese tendieren dementsprechend eher zum Schwachen Physikalismus, der vor allem auch von Byrne und Hilbert vertreten wird.

Die allgemein vorgebrachten Argumente gegen die Notwendigkeitsthese stützen sich auf Beispiele, die plausibel machen sollen, daß die in der aktualen Welt faktisch bestehende Korrelation von Gehalt und Quale unter veränderten Umständen doch auseinandergehen kann. Die Antwort der Repräsentationisten auf diese Einwände folgt der zweifachen Strategien, entweder die metaphysische Unmöglichkeit der Beispiele oder ihre Kompatibilität mit der Notwendigkeitsthese nachzuweisen. Auf die klassischen Fälle und die entsprechenden, ausführlichen Debatten innerhalb der Literatur wird hier jedoch nicht konkret eingegangen werden; aber es scheint auch noch kein entscheidendes Argument gegen den Repräsentationalismus auf der Grundlage dieser Gegenbeispiele gefunden worden zu sein. ${ }^{32}$ Stattdessen soll hier - auch an Beispiel aus der Diskussion über Farben - ganz allgemein überlegt werden, welche Voraussetzungen Gegenargumente gegen die Notwendigkeitsthese erfüllen müssen, wenn diese von einem Schwachen Physikalisten vertreten wird, um dann die Plausibilität einigermaßen einschätzen zu können, inwiefern diese Bedingungen tatsächlich eingehalten werden können. Dabei ist es interessant, daß es bisher wenig Querverbindungen zwischen der Debatte über Qualia einerseits und der Debatte über Farben andererseits gegeben hat, obwohl sie doch vielfältig miteinandern verwoben sind. Und viele der klassischen Argumente werden diese Voraussetzung wohl nicht erfüllen. Vor deren Diskussion ist es jedoch sinnvoll, sich die Notwendigkeitsthese noch einmal in Erinnerung zu rufen:

$32 \mathrm{Zu}$ den bekanntesten Beispielen gehören Qualia-Inversionen oder -Abwesenheiten, Jacksons Wissensargument oder das von Kripke und Levine vorgebrachte Problem der Erklärungslücke (vgl. Abschnitt über Qualia im ersten Teil des Buches). Byrne und Hilbert diskutieren Qualia-Inversion und einige andere Gegenbeispiele, so zum Beispiel welche, die von Peacocke vorgebracht worden sind (vgl. Byrne \& Hilbert (1997c): 267ff.; Fn. 15). 
$\left(\mathrm{NEC}_{\mathrm{NB}}\right)$ Notwendig gilt: ein Gegenstand $\mathrm{x}$ instantiiert die Farbe $F_{\mathrm{i}} \leftrightarrow x$ führt in einem Subjekt $S$ unter Normalbedingungen immer zu Wahrnehmungen mit einem $F_{\mathrm{i}}$-Quale.

Die mit den Farben $F_{\mathrm{i}}$ korrelierten $F_{\mathrm{i}}$-Qualia werden rigide über die Farbqualia der Menschen in der aktualen Welt bestimmt und bleiben in allen Welten dieselben. Dagegen können die Normalbedingungen von möglicher Welt zu möglicher Welt verschieden sein, je nach dem, welche farbwahrnehmenden Subjekte existieren und welche äußeren Umstände vorherrschen. Die letztere Erläuterung schließt somit von vorneherein Gegenargumente aus, die auf Veränderungen der Normalbedingungen für Farbwahrnehmungen aufbauen. Averill formuliert ein solches Beispiel, demzufolge entweder ein bestimmtes Gas in die Atmosphäre der Erde oder eine bestimmte Flüssigkeit in den Bereich zwischen der Linse und der Retina aller menschlichen Augen gelassen wird, die alles Licht im Bereich von 587nm bis $590 \mathrm{~nm}$ absorbiert. Gegenstände, die innerhalb dieses Abschnittes des Spektrums Licht reflektieren, werden dementsprechend ihr Erscheinungsbild für die Menschen mehr oder weniger verändern. Ein Objekt, welches nur in diesem Bereich Licht austrahlt (etwa eine Natriumflamme), wird sogar seine Farbigkeit für uns ganz verlieren. Averill schließt, daß hiermit offensichtlich Situationen vorliegen, in denen die notwendige Korrelation von Quale und repräsentierter Eigenschaft aufgebrochen sind (vgl. Averill (1992): 552ff.).

Doch diese Schlußfolgerung ist vorschnell, da $\left(\mathrm{NEC}_{\mathrm{NB}}\right)$ nur relativ $\mathrm{zu}$ den bestehenden Normalbedingungen gilt. Vertreter einer teleologisch geprägten Gehaltstheorie werden unter Umständen sogar behaupten, daß nach der Veränderung gar keine Normalbedingungen mehr für die fraglichen Subjekte vorliegen, da der neue Zustand der Umgebung nicht mehr dem evolutionär relevanten und für die Herausbildung der Repräsentationsfunktion wesentlichen Habitat (wie es vor dem Wechsel der Bedingungen Bestand gehabt hat) entspricht. ${ }^{33}$ So ist es nicht überraschend, daß die Kor-

33 Es gibt zum Beispiel den Fall von swampman: einem physikalisch und eng-funktional mit einem Menschen identisches Wesen, welches von dem einen Moment zum anderen durch reinen Zufall (etwa durch plötzliche Zusammenballung von Materie) entsteht. Da die mentalen Zustände des swampman keinerlei evolvierte Repräsentationsfunktion aufweisen können (es gibt keine Spezies von swamp- 
relationen nicht mehr erhalten bleiben. In der neuen Umgebung können die für eine andere Umgebung evolvierten Sinnesorgane nicht mehr ihre Funktion ausüben, bestimmte Eigenschaften zu detektieren. Da in dem Fall von Averills Beispiel die Veränderungen vergleichsweise minimal sind, kann jedoch hier davon ausgegangen werden, daß das visuelle System wenigstens noch teilweise seine Repräsentationsfunktion ausüben und sich vielleicht sogar an die neuen, vorherrschenden Bedingungen adaptieren kann.

Doch auch unter dieser Annahme wird die Notwendigkeitsthese ihre Gültigkeit behalten können. Da die Veränderung die äußeren Umstände oder die Beschaffenheit des Subjektes im allgemeinen betrifft, ist es zu erwarten, daß dann damit auch die Etablierung neuer Normalbedingungen verbunden ist. Unter anderen Normalbedingungen werden natürlich aber auch andere Eigenschaften repräsentiert, da die Repräsentationsrelation als nomologische Korrelation unter Normalbedingungen $\mathrm{zu}$ verstehen ist. Während vorher die Farbwahrnehmungstypen mit den das gesamte wahrnehmbare Spektrum betreffenden Reflektanzeigenschaften durch Gesetzmäßigkeiten verbunden gewesen sind, können sie es nach dem Wechsel der Normalbedingungen nur noch mit Reflektanzvermögen sein, deren physikalische Beschreibungen den Bereich zwischen $587 \mathrm{~nm}$ und $590 \mathrm{~nm}$ aussparen. Über diesen Abschnitt kann das visuelle System zwar gar keine Information mehr enthalten, doch es hat unter der neu bestehenden Norm auch gar nicht mehr die Funktion dazu (wenn es, wie gesagt, überhaupt noch die Funktion hat, etwas zu detektieren).

Wenn also die in der aktualen Welt bestehenden Normalbedingungen nicht mehr gegeben sind, dann liegt entweder gar keine Repräsentation vor, da es keine Normalbedingungen mehr gibt; oder die alten sind durch neue ersetzt worden, so daß eine veränderte Repräsentationalität das Resultat ist. In beiden Fällen ist es für den Repräsentationisten sogar zu erwarten, daß die Phänomenalität der Farbwahrnehmungen sich auch ändert, da sie not-

men, keine evolutionären Vorfahren, auf dessen Entwicklung sich diese Funktionalität begründen ließe), repräsentieren sie auch - laut teleogisch (oder historisch-kausal) orientierten Gehaltstheorien - nicht (jedenfalls nicht zu dem Zeitpunkt direkt nach ihrer Entstehung). Für jemanden, der Repräsentation nicht über die evolutionär entwickelte Repräsentationsfunktion bestimmt, ist es natürlich möglich, für den swampman dieselben Normalbedingungen wie für sein menschliches Duplikat anzunehmen, so daß die Zustände beider gleichermaßen repräsentieren (vgl. Tye (1995): 153ff.). 
wendigerweise mit den repräsentierten Eigenschaften zusammenhängt und diese gewechselt haben. Averills Beispiele können folglich die Gültigkeit der Notwendigskeitsthese nicht erschüttern (vgl. Tolliver (1994): 419f.). Dieser Einfluß der Normalbedingungen auf die Repräsentationalität wird deswegen oftmals übersehen, da meistens die Notwendigkeitsthese als (NEC) formuliert wird, derzufolge Gehalt und Quale notwendigerweise zusammenhängen, während gleichzeitig die dahinterstehende, externalistische Gehaltstheorie, welche eben nur unter Normalbedingungen eine nomologische Korrelation zu den repräsentierten Eigenschaften fordert, stillschweigend vorausgesetzt wird (vgl. etwa Byrne \& Hilbert (1997c): 267).

Aus demselben Grund können auch nicht Gegenbeispiele konstruiert werden, die eine veränderte Sensitivität der Rezeptoren im Auge betreffen. So muß der Notwendigkeitsobjektivist keineswegs davon ausgehen, daß Primaten oder andere Tiere die meisten Gegenstände mit exakt derselben Farbphänomenalität wie der Mensch wahrnehmen müssen. Denn da diese Lebewesen entweder gar keine Trichromaten sind, oder aber eine veränderte Lichtempfindlichkeit der drei Rezeptorarten sowie ein anderes wahrnehmbares Spektrum aufweisen, werden sie auch leicht verschiedene Reflektanztypen repräsentieren, da diese, wir wir gesehen haben, mittels $\left(\mathrm{D}_{\mathrm{vs}}\right)$ subjektrelativ, das heißt, über den Output des betreffenden, visuellen Systemes bestimmt werden. Verschiedene proximale Reize können so mit verschiedenen, repräsentierten Eigenschaften zusammenhängen. Für den Starken Physikalismus besteht dieser Ausweg dagegen nicht, weil er Farben nicht subjektrelativ, sondern physik-immanent spezifizieren möchte. Demnach ist er dazu gezwungen, will er einen Objektivismus ${ }_{\mathrm{NEC}}$ vertreten, Farbwahrnehmungen auf Menschen zu reduzieren: worin offensichtlich eine weitere Motivation begründet liegt, als Notwendigkeitsobjektivist für den Schwachen Physikalismus zu optieren.

Dementsprechend können auch keine Änderungen der internen Verarbeitung der einkommenden Signale eine Entscheidung gegen die Notwendigkeitsthese herbeiführen. Es ist sicherlich denkbar, daß es menschliche Subjekte geben kann (oder könnte), die zwar über exakt dieselben Augen verfügen wie wir, deren visuelles System sich aber durch eine andere Gegenfarbtheorie funktional beschreiben läßt. So ist es zum einen möglich, daß die Vorfaktoren der einzelnen Kodierungen sich verändern (wenn zum Beispiel statt $L-M$ auf einmal $L-\mathrm{a} \times M$ mit a $\geq 1$ zutreffen würde; zudem 
weisen auch unsere Informationskanäle nur annähernd eine vorfaktorenfreie Kodierung auf - vgl. Hardin (1988): 34); und zum anderen gibt es noch eine vierte, prinzipielle Weise, die drei Signale miteinander zu koppeln, die in unserem System faktisch nicht realisiert ist: und zwar $L-M-S .{ }^{34}$ Doch beide Arten der Veränderungen des visuellen Systemes führen auch zu einem Wechsel der durch dieses System repräsentierten Reflektanztypen, weil diese mittels $\left(\mathrm{D}_{\mathrm{vs}}\right)$ systemrelativ analysiert werden: und zwar über die Output-Zustände der Informationskanäle des visuellen Systemes (gleiches ergibt sich für die Bestimmung $\left(D_{L}\right)$, da auch diese wesentlich auf die durch die Gegenfarbtheorie beschriebenen Kodierungen Bezug nehmen muß). Und wiederum ist der Starker Physikalismus gezwungen, zur Aufrechterhaltung von $\left(\mathrm{NEC}_{\mathrm{NB}}\right)$ Farbensehen auf bestimmte Subjekte zu begrenzen. Doch diesmal muß die Einschränkung soweit gehen, daß nur noch Subjekte zugelassen sind, die - funktional betrachtet - dasselbe visuelle System aufweisen, welches die Menschen aktual besitzen. Wenn man also davon ausgehen möchte, daß auch andere Lebewesen (insbesondere sehr menschenähnliche) Farben sehen können oder könnten, dann ist die Konjunktion eines Starken Physikalismus mit der Notwendigkeitsthese nicht mehr möglich.

Aber es gibt noch interessantere Fälle, die einem Notwendigkeitsobjektivisten eventuell mehr Schwierigkeiten bereiten werden als die vorhergehenden. Ein einfaches Beispiel ist bereits im Zusammenhang mit der Dispositionalität und der Rigidizität der Farben betrachtet worden. Dort hat sich die Frage gestellt, was mit der Farbdisposition eines Gegenstandes passiert, wenn die relevante Naturgesetzmäßigkeit, welche die Basis der Disposition normalerweise mit ihrer Wirkung verbindet, auf einmal keinen Bestand mehr hat, aber alle anderen Faktoren - wie die (sonstigen) Normalbedingungen oder die Beschaffenheiten des Objektes sowie des wahrnehmenden Subjektes - konstant bleiben. Folgt man der Rigidizitätsintuition, dann wird der Gegenstand seine Farbigkeit behalten,

34 Es gibt die vier Kodierungsmöglichkeiten $L+M+S$ (achromatisches Signal), $L$ $-M+S$ (Rot-Grün-Signal, wobei der sehr geringe $S$-Faktor meistens vernachlässigt wird - vgl. Hardin (1986): 34), $L+M-S$ (Blau-Gelb-Signal) und eben $L-$ $M-S$ (nicht realisiert). Für die Gegenfarbtheorie spielt dabei ein genereller Vorzeichenwechsel der Kodierungen keine Rolle $(L+M-S$ entspricht somit $S-M$ $-L)$. 
weil seine intrinsischen Eigenschaften sich nicht verändert haben. Doch die mit der disjunktiven Basismenge identische Disposition kann dann nicht mehr korrekt repräsentiert werden, da die ursprüngliche Gesetzmäßigkeit ein wesentlicher Bestandteil der Normalbedingungen für die Repräsentation der fraglichen Disposition gewesen ist. Es liegt also genau der gleiche Fall vor wie bei dem Beispiel von Averill. Entweder haben sich durch den Wechsel in der Naturgesetzmäßigkeit auch die Normalbedingungen hinsichtlich der Farbe verändert, und dann ist der damit verbundene Phänomenalitätswechsel nicht mehr überraschend; oder die Normalbedingungen sind trotz Veränderung der Naturgesetze konstant geblieben, aber es kann keine veridische Repräsentation der Farb disposition mehr vorkommen, da überhaupt keine Manifestierung derselben mehr möglich ist und sie somit gar nicht mehr wahrgenommen werden kann.

Bei beiden Alternativen bleibt die Gültigkeit der Notwendigkeitsthese bestehen, so daß der Objektivismus ${ }_{\mathrm{NEC}}$ auch solche Beispiele erklären kann. Nun sind bisher eine große Anzahl von möglichen Gegenbeispielen hinsichtlich der Notwendigkeitsthese zurückgewiesen worden, und zwar solche, die ihre argumentative Kraft auf Veränderungen von einem oder mehreren der folgenden Faktoren stützen: (i) den externen oder internenen Normalbedingungen; (ii) der funktionalen Beschaffenheit des visuellen Systemes (das heißt, der proximalen Reize); (iii) der für die Manifestierung der Disposition relevanten Naturgesetzmäßigkeiten. Ebenfalls ausgenommen sind (trivialerweise): (iv) Fälle, in denen sich die distalen Reize - das heißt, die relevanten, intrinsischen Beschaffenheiten der Gegenstände, welche die Farbdispositionen realisieren - verändern. In allen Fällen bringt eine phänomenale Differenz auch immer einen Gehaltsunterschied mit sich (und umgekehrt).

Jedesmal läßt sich dieser Umstand allein auf die durch $\left(\mathrm{D}_{\mathrm{vs}}\right)$ bestimmte und über die disjunktive Basismenge analysierte Dispositionalität der Farben zurückführen. Denn die einzelnen Disjunkte der mit der Farbdispositionen identischen Disjunktionen werden über ihre kausalen Wirkungen unter Normalbedingungen auf den Output des menschlichen visuellen Systemes individuiert. Veränderungen der hierin involvierten Faktoren führen entweder dazu, daß gar keine Repräsentation von Farbdispositionen mehr vorliegen kann (wenn nur in der aktualen Umgebung eine Repräsentations- 
funktion erfüllt werden kann), oder dazu, daß auf einmal andere Farbdispositionen repräsentiert werden (die jeweils unter den neuen Bedingungen normalerweise $\mathrm{zu}$ bestimmten Wirkungen führen). Und in beiden Fällen verändert sich der Gehalt der Farbwahrnehmungen: entweder gibt es keinen mehr, oder er beinhaltet andere Eigenschaften. Ein Unterschied in der Phänomenalität ist laut $(\mathrm{NEC})_{\mathrm{NB}}$ ) also zu erwarten. ${ }^{35}$

Somit bleibt eigentlich nur eine Art von Beispielen übrig, die möglicherweise die Notwendigkeitsthese widerlegen können: und zwar diejenigen, die den nomologischen Übergang von Kodierungszuständen des visuellen Systemes zu den entsprechenden Farbwahrnehmungen betreffen. Für den Schwachen Physikalismus behauptet die These $\left(\mathrm{NEC}_{\mathrm{NB}}\right)$, daß die betreffenden Gesetzmäßigkeiten notwendig bestehen. Demnach kann es niemals der Fall sein, daß ein funktionaler Output-Zustand $V S_{\text {ROT }}$ (wie immer er auch cerebral realisiert ist) unter Normalbedingungen nicht $\mathrm{zu}$ einer Wahrnehmung mit einem Rotquale führt. Und dies gilt für alle farbwahrnehmenden Subjekte, deren Bewußtsein dieselben, funktionalen OutputZustände von ihrem visuellen System geliefert bekommen können wie unser Bewußtsein von unserem visuellen System. Diese Einschränkung spiegelt sich in der Konsequenz des Schwachen Physikalismus wider, daß ein Gegenstand für jede farbensehende Spezies aller Voraussicht nach eine andere Farbe hat und somit eine Multiplizität der Farbigkeit möglich ist. Doch die Notwendigkeitsthese behauptet noch mehr: es ist ebenso unmöglich, daß ein von $V S_{\text {ROT }}$ verschiedener funktionaler Zustand eines beliebigen visuellen Systemes unter Normalbedingungen zu einer rot-phänomenalen Wahrnehmung führt. Denn laut $\left(\mathrm{NEC}_{\mathrm{NB}}\right)$ gilt unter Normalbedingungen: Subjekt $S$ hat $V S_{\text {ROT }} \leftrightarrow S$ hat eine Wahrnehmung mit einem Rotquale (wobei beide Entitäten rigide über aktuale Menschen definiert werden).

Gegen beide Teilthesen, das heißt, gegen beide Richtungen dieses Bikonditionals könnte nun argumentiert werden. Doch über bloße Spekulationen werden diese Argumentationen wohl im Moment noch nicht herauskommen. Zum einen ist es vorstellbar, daß Primaten oder auch einige unse-

35 Viele der klassischen Beispiele gegen die Notwendigkeitsthese können so von dem Schwachen Physikalismus, der Farbdispositionen mithilfe von $\left(\mathrm{D}_{\mathrm{vS}}\right)$ charakterisiert, zwar nicht widerlegt, dafür aber innerhalb ihrer Position erklärt werden (vgl. Tye (1995): Kap. 7). Darin kann ein Vorteil des Repräsentationalismus gegenüber dem Funktionalismus liegen. 
rer direkten, evolutionären Vorfahren, deren visuelles System sich von unserem unterscheiden wird, trotzdem eine der unsrigen sehr ähnliche Phänomenalität aufweisen oder aufgewiesen haben könnten, wobei diese Ähnlichkeit sich soweit erstrecken könnte, daß sie unter anderem exakt denselben phänomenalen Charakter erleben würden, wie ihn ein phänomenal bewußtes Rotquale für uns zeigt. Dann wäre es unter Normalbedingungen zumindest möglich, daß zwar ein phänomenaler Zustand im Geist, aber nicht der entsprechende, funktionale Output-Zustand im visuellen System vorläge (und somit auch nicht dieselbe Farbdisposition repräsentiert würde). Doch diese Überlegungen lassen sich objektiv wohl kaum überprüfen, da es dafür bereits faktisch möglich sein müßte, phänomenales Bewußtsein empirisch zu erfassen. Doch gerade in der Debatte um die Notwendigkeitsthese geht es ja um die konkrete Frage nach einer solchen, von den Repräsentationisten vorgeschlagenen Möglichkeit, so daß eine mögliche Klärung dieser Frage unabhängig von der Qualiadebatte dieselbe auf einmal überflüssig sein lassen würde: das legt die Vermutung nahe, daß die Debatte um die Notwendigkeitsthese zwar durch solche Gedankenexperimente geordnet, aber letztlich nicht geklärt werden kann.

Andersherum kann man sich ein Inversionsbeispiel denken, in welchem die Kodierungen unseres visuellen Systemes bei dem Übergang ins Mentale nicht wie faktisch bei uns, sondern genau invertiert ,interpretiert“ würden, so daß unter Normalbedingungen $V S_{\text {ROT }}$ immer zu einem Grünquale und $V S_{\text {GRÜN }}$ immer zu einem Rotquale führte. Dabei ist es jedoch alles andere als klar, was sich konkret in unserem Gehirn ändern müßte oder könnte, um solch eine Inversion zu realisieren. Ob die Notwendigkeitsthese gilt oder nicht, bleibt vor dem Hintergrund von Gegenbeispielen eine bloße Frage der Spekulation. Sowohl die Fürsprecher als auch die Gegner der These können die bisher vorgebrachten Beispiele wohl innerhalb ihrer Theorien erklären. Dagegen kann eine solche Debatte dabei hilfreich sein, die Differenzen zwischen den Positionen der Repräsentationalisten und der Phänomenalisten deutlicher herauszuarbeiten und eventuelle Hintergrundsannahmen aufzudecken. Die eine oder die andere Position kann dann aus anderen Gründen abgelehnt werden: wie auch hier schließlich gegen $\left(\mathrm{NEC}_{\mathrm{NB}}\right)$ und den Repräsentationalismus entschieden wird, da der ObjektivismuS $_{\mathrm{NEC}}$ die reichhaltige Phänomenalität der Farben nicht über die repräsentierten Reflektanzeigenschaften erklären kann. Und darüberhinaus soll- 
te klar sein, daß, wenn es sich in Zukunft doch herausstellen sollte, daß es allein logisch möglich ist, daß Gehaltsunterschiede und Qualiadifferenzen nicht unbedingt immer in Deckung sein müssen, dann die Notwendigkeitsthese nicht mehr aufrechterhalten werden kann. ${ }^{36}$

Zusammenfassend läßt sich folgendes sagen. Der Starke Physikalismus wird die Notwendigkeitsthese wohl nicht annehmen können; es sei denn, er würde Farbensehen nur auf solche Subjekte beschränken, die aus biologisch-funktionaler Sicht exakt das gleich visuelle System besitzen, wie es uns Menschen faktisch zukommt. Vor dem Hintergrund unserer Intuitionen und naturwissenschaftlichen Erkenntnisse über die visuellen Wahrnehmungen von Tiere, oder auch von unseren direkten Vorfahren, stellt dies jedoch eine wenig überzeugende und möglichst zu vermeidende Konsequenz dar. Zudem müßte $\left(\mathrm{NEC}_{\mathrm{NB}}\right)$ auf ähnliche Weise modifiziert werden wie die Aktualitätsthese. Der Schwache Physikalismus hat indessen zur Zeit noch keine Probleme, die Gültigkeit von $\left(\mathrm{NEC}_{\mathrm{NB}}\right)$ anzunehmen, wenn er Dispositionen als Disjunktionen und die relevanten Naturgesetzmäßigkeiten als Bestandteil der Normalbedingungen ansieht. Die Subjektrelativität der Farben ermöglicht es dem Schwachen Physikalismus - im Gegensatz zum Starken, der von der stärkeren Physik-Immanenz ausgeht -, auch auf einfache Weise auf die Subjektrelativität der Farbphänomenalität zu schließen. Das noch fehlende Glied - der Übergang von Zuständen des visuellen Systemes zu mentalen Zuständen - ist von seiner Natur aus derart mysteriös, daß bisher außer Spekulationen nichts gegen oder für die Akzeptanz von $\left(\mathrm{NEC}_{\mathrm{NB}}\right)$ durch einen Schwachen Physikalismus gesagt werden kann. Als objektivistische Alternative steht zudem immer noch der Primitivismus zur Verfügung. Für einen Subjektivismus dagegen macht die Diskussion um die Notwendigkeitsthese hinsichtlich von Farben (wohl aber hinsichtlich von Reflektanzeigenschaften) gar keinen richtigen Sinn, da er von vorneherein von einer logisch-begrifflichen Verbindung zwischen Farben und Farbqualia ausgeht.

36 Beispielsweise hat Shoemaker (während einer Diskussion im Mai 1998) die Meinung vertreten, daß solche Fälle in beiden Richtungen prinzipiell möglich sein müßten; und die logische Möglichkeit würde bereits genügen, um $\left(\mathrm{NEC}_{\mathrm{NB}}\right)$ ablehnen zu müssen. 


\subsubsection{Probleme mit der Erklärung der Phänomenalität}

Der Objektivismus $_{\mathrm{NEC}}$ - in Form des Schwachen Physikalismus - hat es sich zum Ziel gesetzt, die Repräsentiertheit und die Phänomenalität der Farben miteinander zu vereinen. Ganz konkret handelt es sich hierbei um das Vorhaben von Byrne und Hilbert, die eine solche Farbtheorie vertreten, sowie von den Repräsentationalisten, die sich auf die Schriften ersterer berufen (vgl. Hilbert (1987); Byrne \& Hilbert (1997c); Tye (1995):146). Dieser Abschnitt wird diesen Versuch, die Transparenzthese (TT) aufrechtzuerhalten, kritisch untersuchen, wobei davon ausgegangen werden soll, daß die Notwendigkeitsthese (NEC) gültig ist (mit Ausnahme vielleicht von dem - relativ unwichtigen - Merkmal der Einfachheit). Die phänomenalen Merkmale und Beziehungen, die den phänomenalen Aspekt der Intuitiven Farbkonzeption ausmachen, sind nun wesentlich Eigenschaften des phänomenalen Charakters der Farbwahrnehmungen: sie sind also für die Farbqualia konstitutiv. Möchte man nun diese Phänomenalität auf die objektiv verstandenen Farben übertragen, so gibt es zwei Möglichkeiten, die den beiden Ausformungen des Repräsentationalismus entsprechen: die Farbqualia sind entweder mit den repräsentierten Farben identisch (wie es Dretske bevorzugt); oder sie werden mit den repräsentationalen Eigenschaften der Wahrnehmungen gleichgesetzt, welche wiederum allein über die repräsentierten Eigenschaften spezifiziert sind (wie es etwa Tye gesehen haben möchte). Das heißt, Qualia werden mit dem allgemeinen Gehalt oder mit der Eigenschaft, einen solchen Gehalt zu besitzen, identifiziert.

Abgesehen von theoretischen Unterschieden hinsichtlich der Introspektion mentaler Zustände (beide möchten Qualia als introspektiv zugänglich ansehen, doch Dretske leugnet die Introspektierbarkeit repräsentationaler Eigenschaften von Wahrnehmungen) unterscheiden sich diese beiden Varianten wohl nur in der Verwendung des Ausdruckes „Qualia“. Auch für eine Ontologie der repräsentierten Farben ist diese teils substantielle, teils terminologische Differenz unerheblich, so daß zwischen den beiden Varianten im weiteren Verlauf der Diskussion nicht mehr unterschieden werden muß. Bevor die möglichen Lösungsvorschläge für das Problem, Objektivität und Phänomenalität $\mathrm{zu}$ vereinbaren, betrachtet werden sollen, ist es wichtig, darüber klar zu werden, was ein Objektivismus $\mathrm{SEC}_{\mathrm{NEC}}$ - geht man von einer notwendigen Beziehung zwischen Farben (oder Gehalt) und Farbqualia aus 
- überhaupt zu leisten hat, damit die objektiven, repräsentierten Farben wirklich auch als phänomenale Eigenschaften im Sinne der These (PHE) gelten können. Dies kann offensichtlich nur dann der Fall sein, wenn die mit den Farben identifizierten Eigenschaften $P$ zwei Bedingungen erfüllen: (a) sie müssen untereinander exakt dieselben Ähnlichkeits- und Verschiedenheitsrelationen aufweisen wie die Farbqualia selbst; (b) und sie müssen zudem Merkmale zweiter Ordnung zeigen, die der Reinheit oder Gemischtheit sowie den Inkombatibilitäten der Farbqualia genau entsprechen. Eine ähnliche Bedingung findet sich auch bei Johnston formuliert, der diskutiert, wie ein Objektivismus generell dem Umstand gerecht werden kann, daß diese phänomenale Merkmale laut (PHE) als Bestandteil der Natur der Farben angesehen werden. ${ }^{37}$

„[The unity principle for canary yellow is that - der Autor] thanks to its nature and the nature of teh other determinate shades, canary yellow, like the other shades, has its own unique place in the network of similarity, difference and exclusion relations exhibited by the whole family of shades. (Think of the relations exemplified along the axes of hue, saturation and brightness in the so-called color solid...). [...] The friend of the [objectivist - der Autor] account should be allowed to answer that indeed it is not a matter of scientific discovery that canary yellow is not a shade of blue. Rather, he might say, such a principle, along with other unity principles, must be held true as a condition on any family of properties deserving the color names. So the principle that canary yellow is not a shade of blue turns out to be relatively a priori after all. More exactly what is a priori is a biconditional: $\mathrm{P}$ deserves the name 'canary yellow' just in case (i) $\mathrm{P}$ is the ... surface property standardly responsible for the appearances as of canary yellow things and (ii) this property stands in the right similarity relations to other standardly explanatory ... properties. On the envisaged account, a given property turns

37 Johnston bringt gegen den Objektivismus ${ }_{\mathrm{NEC}}$ ein Argument an, daß sich sehr stark an dem Sichtbarkeitsargument gegen den Objektivismus ${ }_{\mathrm{ACT}}$ orientiert. Wenn die für die Farben konstitutiven Ähnlichkeitsrelationen zwischen den physikalischen Reflektanzeigenschaften bestehen, dann können wir, so schließt Johnston, nur mithilfe der Empirie feststellen, welche Farbe (das heißt: welche Reflektanzeigenschaft) ein gegebener Gegenstand genau hat (vgl. Johnston (1992): 150ff.). Das ist aber nur dann richtig, wenn man - wie Johnston - voraussetzt, daß physikalische Eigenschaften nicht sichtbar sein können: Doch dies erfordert eine Wahrnehmungstheorie im Geiste der Sinnesdatentheorie, so daß der Einwand hier vor dem Hintergrund des Direkten Realismus vernachlässigt werden kann. 
out to count as canary yellow only if a complex similarity condition on that property and a host of others is dicovered to hold.“ (Johnston (1992): 138; 150)

Die Repräsentationalisten wählen genau die hier beschriebene Strategie. Die objektiven, von der Colorimetrie zu identifizierenden Eigenschaften $P$ kommen nur dann als Farben in Frage, wenn sie einerseits repräsentiert sind (Johnstons Bedingung (i)) und andererseits in den erwähnten Ähnlichkeits- und Verschiedenheitsrelationen zueinanderstehen (Johnstons Bedingung (ii) und meine Bedingung (a)); zudem müssen sie noch die beschriebenen Eigenschaften zweiter Ordnung besitzen (meine Bedingung (b)). Der Forderung nach der Repräsentiertheit kommt der ObjektivismuS $\mathrm{NEC}_{\mathrm{N}}$ nun dadurch nach, daß er Farben im Sinne des Schwachen Physikalismus als Reflektanztypen $S S R_{\mathrm{T}}$ ansieht. Bleiben also noch die Bedingungen (a) und (b), die von den Reflektanzeigenschaften erfüllt werden müssen. Es lassen sich bei den Schwachen Physikalisten, die gleichzeitig die Notwendigkeitsthese akzeptieren, zwei Lösungsvorschläge für dieses Problem entdecken. Der erste Theorieentwurf versucht, zur phänomenalen Struktur der Farbqualia eine einfache Entsprechung innerhalb der Klasse der Reflektanztypen identifizieren zu können, während der zweite den Schwierigkeiten mit der Einführung von vielen verschiedenen, repräsentierten Farbdeterminablen begegnen möchte. Es wird sich jedoch zeigen, daß beide Alternativen nicht zum gewünschten Ziel führen können (vgl. K. Campbell (1993): 259ff.).

Die Grundidee des ersten Erklärungsversuches, der sich in Hilberts Buch Color and Color Perception (1987) findet, ist, daß die den Farbqualia zukommenden phänomenalen Merkmale bestimmte, diesen entsprechende Merkmale der Reflektanztypen repräsentieren. Diese Repräsentation ist dabei prinzipiell möglich, weil die Akzeptanz der Notwendigkeitsthese es mit sich bringt, daß Qualia als eine besondere Art von repräsentationalem Gehalt analysiert werden können. Die phänomenalen Ähnlichkeiten und Verschiedenheiten repräsentieren demnach isomorphe, physikalische Ähnlichkeiten und Verschiedenheiten unter den Reflektanzeigenschaften; und die Reinheit oder Gemischtheit einer Farbe zeigt eine objektiv bestimmbare „Reinheit" oder „Gemischtheit" der betreffenden Reflektanzen an. Trifft dies tatsächlich zu, dann sind die Bedingungen (a) und (b) erfüllt. Zudem werden die Ähnlichkeitsrelationen es mit sich bringen, daß 
dem von Johnston erwähnten phänomenalen Raum der Qualia ein isomorpher, physikalischer Farbraum der Reflektanztypen oder -tripel entsprechen wird.

„If we think of triples of integrated reflectances as coordinates in a three-dimensional space, then similar colors will occupy adjacent regions of that space. I will call the space defined in this way color space." (Hilbert (1987): 117)

Beschränkt man sich allein auf den Farbton, wird dieser Ähnlichkeitsraum für die objektiven Farben - wie auch für die Farbqualia - eine Kreisform aufweisen. Wie der Farbton Orange dem reinen Rot ähnlicher ist als dem reinen Blau, so wird auch der Reflektanztyp $S S R_{\text {ORANGE }}$ dem Typ $S S R_{\text {ROT }}$ ähnlicher sein als dem Typ $S S R_{\text {BLAU }}$. Doch zum einen bleibt diese Theorie wesentlich unvollständig, da sie nichts zu den in Bedingung (b) genannten Merkmalen zweiter Ordnung zu sagen hat. Und zum anderen ist es möglich, gegen diese einfache Lösung, die Bedingung (a) zu erfüllen, drei Gegenargumente $\mathrm{zu}$ formulieren, die im folgenden einzeln kurz dargestellt werden sollen. ${ }^{38}$ Der erste Einwand zielt auf den Umstand ab, daß es zwar ohne weiteres möglich sein wird, die objektiven Farben in einem dem phänomenalen Raum der Qualia isomorphen Raum darzustellen, daß dieser so gewonnene Farbraum dadurch jedoch noch nicht automatisch physikalisch beschreibbar sein wird. Isomorphie und Physikalität können also nicht gleichzeitig gewährleistet werden. Dahinter verbirgt sich die Tatsache, daß sich wohl zu jeder vorgebenen Menge von Entitäten mit Ähnlichkeitsbeziehungen auch eine mathematische Topologie finden läßt, die diese Ähnlichkeiten genau abbildet. So ist es nicht überraschend, daß auch die Reflektanztypen auf eine ähnliche Weise mathematisch angeordnet werden können, wie es der Farbkreis vorgibt. ${ }^{39}$ Das Problem für den Repräsentationalisten ist nun jedoch, daß diese mathematisch orientierte Anordnung keine physikalische Interpretation erhalten kann. Es sind bisher noch keine physikalisch beschreibbaren Merkmale der Reflektanztypen

38 Die drei Einwände sind ursprünglich von Frank Hofmann in einem unveröffentlichten Manuskript vorgebracht worden.

39 Dies geschieht mithilfe der Kodierungen der Gegenfarbtheorie, die sich der quantitativen Verhältnisse zwischen den Intensitätswerten für die drei Wellenlängenbereiche $L, M$ und $S$ bedient. 
SSR ORANGE$_{\text {, }}$ SSR $_{\text {ROT }}$ und SSR $_{\text {BLAU }}$ identifiziert worden, die die oben beschriebenen Ähnlichkeitsrelationen erklären könnten; und es ist wohl auch nicht zu erwarten, daß dies in der Zukunft geschehen wird.

Ein ähnlicher Einwand findet sich bereits in einigen anderen Kritiken der objektivistischen Position vorgezeichnet (vgl. etwa K. Campbell (1993): 259; 262ff.). Er kann übrigens auch nicht dadurch zurückgewiesen werden, daß Reflektanztypen keine physik-immanenten Eigenschaften sind. Denn es läßt sich wahrscheinlich generell keine rein objektive, qualitative Interpretation der entsprechenden, mathematisch-quantitativ beschriebenen Ähnlichkeiten zwischen den einzelnen Reflektanzeigenschaften angeben: ganz unabhängig davon, ob die Objektivität bloß Physikalität oder auch Physik-Immanenz beinhaltet. So ist es nicht allzu überraschend, daß Byrne und Hilbert in ihrem neueren Aufsatz zu demselben Resultat kommen und die in Hilberts Buch vorgeschlagene Lösung nicht mehr vertreten.

\begin{abstract}
„We need not pursue this any further, because if physicalism (our brand of it, at least) is true, some intuitively correct natural similarity claims... will be false. Perhaps SSR $_{\text {RED }}$ is more similar, in the natural sense, to SSR ORANGE $_{\text {than }}$ to SSRGREEN; but it seems extremely doubtful that this relation holds between, for example, SSR $_{\text {BLUE }}$, SSR $_{\text {PURPLE }}$ and SSR GREEN. $_{\text {. }}$

[In Hilbert (1987)], it is claimed that a certain space of such triples, with a physically motivated metric, is roughly isomorphic to color similarity space. But this is not right, or not right enough: the space of triples provides only a very loose approximation to similarity relations among colors.“" (Byrne \& Hilbert (1997c): 276; Fn. 32)
\end{abstract}

Das zweite Gegenargument ist nun, daß der Objektivismus ${ }_{\mathrm{NEC}}$ Zwar davon ausgehen kann, daß die Reflektanztypen von den Wahrnehmungen repräsentiert werden, nicht aber unbedingt, daß dies ebenso für deren Eigenschaften und Relationen zweiter Ordnung gelten wird. Und sind letztere nicht im Gehalt mit enthalten, dann können sie auch keinen Einfluß auf die phänomenalen Beziehungen zwischen den Farbqualia haben. Dahinter verbirgt sich die Idee, daß es nicht ganz so einfach ist, den Gehalt eines mentalen Zustandes so einfach durch eine zusätzliche Eigenschaft zu erweitern. Ob dieser Einwand aber zutrifft, ist jedoch nicht so klar, da der Repräsentationalist vielleicht davon ausgehen kann, daß solch eine Erweiterung gar 
nicht erforderlich ist, um die Repräsentation der Ähnlichkeitsrelationen zu gewährleisten. Denn vorausgesetzt, daß es eine physikalische Interpretation des objektiven Farbenraumes gibt, ist es doch gut denkbar, daß die entsprechenden Ähnlichkeitsrelationen interne Relationen darstellen und somit sich direkt aus den intrinsischen Beschaffenheiten der Farben ergeben. Und in diesem Fall wird es fraglich, ob zusätzlich zu den Repräsentationen der Farbeigenschaften erster Ordnungen wirklich noch Repräsentationen von den Ähnlichkeitsrelationen zweiter Ordnung erforderlich sind. Reicht es nicht aus, die Größe zweier Gegenstände wahrzunehmen, um auch gleichzeitig die Tatsache zu repräsentieren, daß der eine größer als der andere ist? Es ist jedenfalls nicht einfach zu sehen, ob dieser Einwand wirklich seine gewünschte Wirkung zeigen kann. Doch

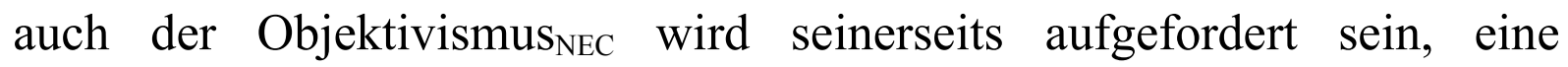
Erklärung darüber abzuliefern, daß die Ähnlichkeiten repräsentiert werden.

Der dritte Einwand stützt sich auf die bereits im Zusammenhang mit den Normalbedingungen erwähnte intersubjektive Variationen in den Lokalisierungen von reinen Farbtönen. So werden zum Beispiel von verschiedenen Betrachtern Gegenstände mit ganz unterschiedlichen Reflektanztypen als Träger des reinen Grüntones gesehen, wobei die einzelnen Abweichungen verhältnismäßig groß sein können (so daß es etwa unter diesen als rein grün beurteilten Objekten auch welche gibt, die von einigen Menschen als rein blau wahrgenommen werden). Hinsichtlich der Bestimmung der Normalbedingungen können diese Abweichungen vielleicht noch als Anzeichen für die relative Unbestimmtheit der Wahrnehmung angesehen und in Kauf genommen werden, zumal die Reinheit und Gemischtheit der Farbtöne aus evolutionärer Sicht relativ unwichtige Charakteristika zu sein scheinen. Für den Objektivismus ${ }_{\mathrm{NEC}}$ steht dieser Weg jedoch wohl nicht offen, da er jede phänomenale Differenz auf einen ganz genau festgelegten, physikalischen Unterschied in den Reflektanzeigenschaften zurückführen können und folglich bei gleichem Gehalt auch ein gleiches Quale vorliegen muß. Doch dies ist wenigstens hinsichtlich der Reinheit und Gemischtheit von Farbtönen nicht gegeben. Der einzige Ausweg wäre, davon auszugehen, daß die meisten, die einen Gegenstand als rein grün sehen, genau genommen einer Fehlrepräsentation (hinsichtlich der Reinheit) unterliegen eine Konsequenz, die besser vermieden werden sollte. Damit kann der erste Erklärungsversuch des Objektivismus ${ }_{\mathrm{NEC}}$ wohl zurückgewiesen werden. 
In ihrem gemeinsam geschriebenen Aufsatz entwickeln Byrne und Hilbert einen anderen Lösungsvorschlag, auf der Idee beruht, daß Farbwahrnehmungen nicht nur einen, sondern gleich mehrere repräsentationale Gehalte haben (vgl. Byrne \& Hilbert (1997c): 274ff.; 279ff.). Dabei greifen sie auf den Umstand zurück, daß sich zwischen der ganz allgemeinen Determinable Farbe (oder Farbigsein) und deren einzelnen, maximal bestimmten Determinanten sich eine Vielzahl von weniger allgemeinen Determinablen (die gleichzeitig als weniger bestimmten Determinanten für die höherliegenden Determinablen fungieren können) finden lassen. Ein Briefkasten instantiiert folglich nicht nur den maximal bestimmten Farbton Gelb $_{17}$, sondern auch die weniger bestimmten Eigenschaften Postgelb, Gelb (oder Gelblichkeit) und Farbigsein. Somit entsteht eine hierarchische Ordnung von Determinablen und Determinanten. Ganz zuoberst, an dem Punkt größtmöglichster Allgemeinheit, befindet sich die Determinable Farbigsein, ganz zuunterst die maximal bestimmte Determinante $\mathrm{Gelb}_{17}$, und dazwischen die Eigenschaften Postgelb und Gelb, die hinsichtlich der höhergelegenen Eigenschaften als Determinante und hinsichtlich der tiefergelegenen als Determinable gelten. Wenn ein Gegenstand also ganz bestimmte eine Farbe besitzt, instantiiert er automatisch noch viele, weitere und allgemeinere Eigenschaften. Da letztlich alle anderen Eigenschaften auf den maximal bestimmten Farben supervenieren, stellt diese Vervielfachung der instantiierten Eigenschaften auch kein ontologisches Problem dar.

Diese einzelnen Farbdeterminablen und -determinanten können nun mit verschieden allgemein definierten Reflektanztypen gleichgesetzt werden. Der maximale Reflektanztyp $S S R_{\mathrm{GELB}-17}$ ist folglich ein token des Reflektanztypes $S S R_{\text {POSTGELB }}$, und dieser wiederum der noch allgemeineren Reflektanztypen $S S R_{\text {GELB }}$ (oder $S S R_{\text {GELBLICHKEIT }}$ ) und $S S R_{\text {FARBIGSEIN }}$ (welcher der ganz allgemeinen Definition der Reflektanztypen entspricht). Der Einfachheit halber wird im folgenden jedoch weiterhin von den Farben anstatt von den mit diesen identifizierten Reflektanzeigenschaften die Rede sein. Byrne und Hilbert konzentrieren sich nun auf vier besondere Superdeterminablen, die sich in der Hierarchie direkt unter der allgemeinsten Determinable Farbigsein befinden: und zwar Rötlichkeit, Gelblichkeit, Grünlichkeit und Bläulichkeit (oder die entsprechend allgemeinen Reflektanztypen). Unter die jeweiligen Superdeterminablen fallen nun unzählig viele, maximal bestimmte Farbtöne (oder $S S R_{\mathrm{T}}$ ). 
Die feingradigst spezifizierten Eigenschaften Zinnoberrot, Purpur, reines Rot, Violet oder auch Orangenfarben und Sonnenuntergangsrot sind alles Determinanten der Superdeterminable Rötlichkeit. Doch in den meisten Fällen gehören sie auch einer anderen Superdeterminablenklasse an: zum Beispiel fällt Orange unter die Gelblichkeit, während Violet so mit der Bläulichkeit zusammenhängt. Die vier den Superdeterminablen entsprechenden Mengen überschneiden sich also. Dabei ist es jedoch eine auf die Gemischtheiten und Inkompatibilitäten zurückzuführende Tatsache, daß eine maximale Determinante mindestens unter eine, aber höchstens nur unter zwei Superdeterminablen zu fassen ist. Die vier reinen Farbtöne sind dabei die einzigen, die jeweils nur eine Superdeterminable ausfüllen. Und es ist nicht möglich, daß ein Farbton etwa sowohl unter die Superdeterminable Rot als auch die Superdeterminable Grün fällt.

An dieser Stelle führen Byrne und Hilbert für Farbwahrnehmungen multiple Gehalte ein. ${ }^{40}$ Wird ein Gegenstand nun als Träger einer maximal bestimmten Farbe repräsentiert, dann hat die entsprechende Wahrnehmung zumindest noch einen zweiten Gehalt, der aus einer oder zwei dieser Superdeterminablen besteht. Ist der Briefkasten beispielsweise von einem reinen Gelbton, dann enthält dieser zusätzliche Gehalt nur die Superdetermimable Gelblichkeit. Zeigt er dagegen einen Gelbton mit einem Rotstich, wird zusätzlich noch die Superdeterminable Rötlichkeit repräsentiert. Auf diese Weise weisen die Repräsentationen von reinen Farben einen Gehaltsunterschied im Vergleich zu den Repräsentationen gemischter Farben auf, der sich ontologisch auf die Anzahl der Superdeterminablen zurückführen läßt, unter die der repräsentierte Farbton fällt. Und der Gehaltsunterschied kann wiederum die phänomenale Differenz erklären. Somit können Byrne und Hilbert in der Welt Unterschiede in den instantiierten, objektiven Farben (oder Reflektanztypen) dafür verantwortlich machen, daß auch ein phänomenaler Unterschied vorliegt: es werden ein verschieden viele Superdeterminablen repräsentiert. Ihre Theorie kann demnach die Bedingung (b) erfüllen.

40 Es scheint dabei im folgenden eigentlich gleichgültig zu sein, ob man von einem Gehalt mit vielen verschiedenen, repräsentierten Eigenschaften oder vielen Gehalten mit jeweils einer repräsentierten Eigenschaft ausgeht: solange klar ist, daß die Sinneserfahrungen mehrere, zudem verschieden feingradig bestimmte Farbdeterminanten repräsentieren. 
Für die Erklärung der Ähnlichkeits- und Verschiedenheitsrelationen benötigen sie jedoch noch - beliebig viele - weitere repräsentierte Determinablen, die weniger allgemein sind als die Superdeterminablen. Will man nun Gegenstände auf ihre Ähnlichkeit überprüfen, beginnt man bei den Superdeterminablen. Teilen sich zwei Objekte eine Superdeterminable, dann sind sie sich ähnlicher, als wenn sie keine Superdeterminable gemeinsam hätten. ${ }^{41}$ Kommt ihnen ein- und dieselbe Superdeterminable zu, dann muß nun hinsichtlich der nächstunteren Ebene gefragt werden, ob sie in einer bestimmten Determinablen übereinstimmen oder nicht. Diese Vergleiche können beliebig weiter fortgeführt werden, bis man schließlich zu den maximal bestimmten Farbtönen kommt. Zwei unterschiedliche, zinnoberrote Farbtöne sind sich somit untereinander ähnlicher als im Verhältnis zu einem Purpurrot, weil sie mit letzterem nur die Superdeterminable Rötlichkeit gemeinsam haben, nicht aber die relativ gesehen spezifischere Determinable Zinnoberrot. Und zu einer grünlichen Farben weisen sie noch weniger (oder gar keine) Ähnlichkeit auf. Nimmt man nun für jede Ebene der Determinablen einen eigenen Gehalt der Farbwahrnehmungen an, können auch die Ähnlichkeiten und Verschiedenheiten zwischen den Farbqualia auf diese ontologischen Gemeinsamkeiten und Differenzen zurückgeführt werden. Der Vorschlag von Byrne und Hilbert erfüllt also auch die Bedingung (a).

Doch auch dieser zweite Versuch, die Phänomenalität der objektiven Farben zu retten, schlägt fehl. Das Hauptproblem stellt dabei die Beschaffenheit der multiplen Gehalte dar. Es wird üblicherweise davon ausgegangen - und die Repräsentationalisten teilen diese Meinung -, daß ein Wahrnehmungsgehalt nicht-begrifflich ist, wobei diese Charakteristik zumindest eine relativ hohe Feingradigkeit impliziert. Dies ist, wie es scheint, durch die Feingradigkeit der in Wahrnehmungen diskriminierbaren und repräsentierbaren Reflektanztypen gewährleistet (daß diese zudem Disjunktionen metamerischer Reflektanzprofile sind, stellt dabei kein Hindernis dar). Aber es ist fraglich, ob dies noch ebenso für die Repräsentation der allgemeineren Determinablen gelten kann. Denn diese zeichnen sich ja gerade

41 Im Grunde ist es fraglich, ob es überhaupt Sinn macht, von einer Ähnlichkeit oder einer Unähnlichkeit zu sprechen, wenn zwei Farbtöne nicht wenigstens eine Superdeterminable gemeinsam haben. Wie soll entschieden werden, ob ein (nicht-bläulicher) Rotton dem einen oder dem anderen Blaugrün ähnlicher ist? 
dadurch aus, daß sie wesentlich weniger genau spezifiziert sind als die Reflektanztypen. Insbesondere auf der Ebene der Superdeterminablen kann nicht mehr eine Feingradigkeit vorliegen, die eine nicht-begriffliche Repräsentation begründen könnte: die Genauigkeit einer Unterteilung der Farbtöne in vier Klassen kann eigentlich gar nicht mehr wirklich mit der Genauigkeit einer Unterteilung in etwa eine Million Farbtöne (soviele, wie Menschen diskriminieren können) verglichen werden. In diesem Sinne kann es sich bei den Repräsentationen der (meisten) allgemeineren Farbdeterminablen nur um begriffliche Repräsentationen handeln. Da die phänomenalen Merkmale und Beziehungen bereits in den nicht-begrifflichen Farbwahrnehmungen gegeben sind, kann auch die zweite, zusammen mit Byrne entwickelte Theorie von Hilbert das gewünschte Ziel erreichen und die Bedingungen (a) und (b) erklären. ${ }^{42}$

Zudem können noch drei weitere Einwände gegen den ObjektivismuS $_{\mathrm{NEC}}$, der diese Lösungsvariante bevorzugt, angeführt werden. Erstens stellt sich erneut das Problem der intersubjektiven Variationen hinsichtlich der Lokalisierung von reinen und gemischten Farbtönen. Es ergeben sich zweitens wahrscheinlich Schwierigkeiten aus dem Umstand, daß die Repräsentation allgemeinerer Determinablen mit der Repräsentation einer disjunktiven Menge aus bestimmten Reflektanztypen identisch ist. Ein Gegenstand, der die maximal bestimmte Farbe Rot ${ }_{1}$ instantiiert, wird somit

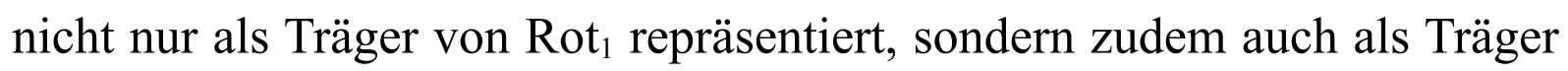
einer allgemeineren Determinable Rot. Da letztere sich aus all den unter sie fallenden Rottönen disjunktiv zusammensetzt, wird somit auch die (vielleicht unendliche) Disjunktion $\operatorname{Rot}_{1} \vee \operatorname{Rot}_{2} \vee \ldots \vee \operatorname{Rot}_{n} \vee \ldots$ repräsentiert. Doch damit wird sich vermutlich das bekannte Diskjunktionsproblem stellen (vgl. etwa Boghossian (1991)). Und schließlich es ist drittens problematisch, daß der Objektivimus $\mathrm{NEC}_{\mathrm{N}}$ keine zufriedenstellende Antwort darauf geben kann, warum es nur vier Superdeterminablen gibt, und warum dar-

42 Hofmann (2002) widerlegt überzeugend verschiedene, dem Objektivismus ${ }_{\mathrm{NEC}}$ eventuell offenstehende Strategien, die Phänomenalität der Farbqualia nicht auf sinnliche, sondern begriffliche Repräsentationen zurückzuführen. Diese Überlegungen können hier jedoch ausgespart bleiben, da in diesem Buch die allgemeine Auffassung geteilt wird, daß die durch (PHE) beschriebenen Merkmale die Natur der Farbqualia mitbestimmen und somit die Diskussion nicht von der sinnlichen auf die begriffliche Ebene verschoben werden kann. 
überhinaus gerade genau diese vier (Vergleichbares gilt natürlich auch für die anderen Ebenen innerhalb der Determinablenhierachie). Faßt man die Rötlichkeit (wie es etwa auch Byrne und Hilbert tun) zum Beispiel als Rotoder-Orange-oder-Violet auf, dann ist die Frage berechtigt, warum Gelboder-Orange-oder-Grün oder auch Gelb-oder-Orange-oder-Gelbgrün-oderGrün nicht ebenfalls Superdeterminablen sein können: denn aus physikalischer Sicht werden sich keine bedeutsamen Unterschiede feststellen lassen. Auch hier fehlt eine physikalische Interpretation für die an der Phänomenalität orientierten Anordnung und Klassifizierung der Reflektanztypen (vgl. K. Campbell (1993): 262f.).

Beide Strategien der Notwendigkeitsobjektivisten sind somit gescheitert. Zwar werden diese natürlich die Möglichkeiten für einen Objektivismus $_{\mathrm{NEC}}$ längst noch nicht ausschöpfen, doch im Moment sind wohl keine besseren oder vielversprechenden Lösungsvorschläge als die zwei behandelten in Sicht. Demnach sollte diese Variante des Farbphysikalismus wenigstens für den Augenblick - nicht als eine konsistente Theorie der Farben betrachtet werden. Damit kann aber auch der (reine) Repräsentationalismus, der den Objektivismus $\mathrm{NEC}_{\mathrm{NEC}}$ impliziert, als angemessene Theorie des phänomenalen Charakters von Wahrnehmungen zurückgewiesen werden (eine repräsentationalistisch-funktionalistischen Alternative deutet Hofmann (2002) an). Für den Farbobjektivisten eröffnen sich nun zwei Möglichkeiten: entweder schwächt er seine Position zu einem Objektivismus ${ }_{\mathrm{ACT}}$ $\mathrm{ab}$ und nimmt die damit verbundenen Konsequenzen in Kauf; oder er stärkt sie derart, daß er dem Problem der Vereinigung von Objektivität und Phänomenalität in den Farben entgeht, indem er diese nicht als physikalisch beschreibbare Eigenschaften analysiert, sondern sie einfach als primitive Eigenschaften in der Welt annimmt. Denn das Hauptproblem für einen Objektivismus $_{\mathrm{NEC}}$ liegt ja nicht in der Akzeptanz der Notwendigkeitsthese, sondern vielmehr in dem Umstand, daß Farben mit physikalisch beschreibbaren Eigenschaften gleichgesetzt werden, die als solche keine der Farbphänomenalität vergleichbare Strukturen zeigen können. Dem Notwendigkeitsobjektivisten bleibt so nur noch die Alternative eines Primitivismus. 


\subsection{Der Primitivismus}

Der Primitivismus geht von der Überzeugung aus, daß Farben genauso sind, wie sie uns erscheinen. Das heißt, die Offensichtlichkeitsthese (OS) gilt im Fall der Farben: Farben sind wesentlich sowohl repräsentierte als auch phänomenale Eigenschaften; und mehr gibt es über Farben nicht zu sagen. Insbesondere wird das Merkmal der Einfachheit der Intuitiven Farbkonzeption durch solche, primitive Farbeigenschaften erfüllt. Sie müssen insbesondere nicht physik-immanent oder subjektrelativ spezifiziert werden. Farben sind primitive Eigenschaften in der Welt. Es ist weder möglich, noch erforderlich, sie durch andere Eigenschaften zu analysieren. Die Analysethese (ANA) findet also keine Anwendung; und damit insbesondere auch nicht die These (PHY) des Farbphysikalismus (zudem müssen die von (OS) implizierten (REP) und (PHE) somit direkt über die Farben $F$ formuliert werden). Dies ist in Einklang mit der oben gewonnenen Erkenntnis, daß der Physikalismus die Einfachheit für Farben nicht aufrechterhalten kann. Die Akzeptanz der Offensichtlichkeitsthese bedingt natürlich auch die Annahme der Notwendigkeitsthese (NEC); denn sonst könnte die Phänomenalität nicht den repräsentierten Eigenschaften zukommen (vgl. Einleitung zu Byrne \& Hilbert (1997a): xxiv).

Dabei können die im letzten Abschnitte gegen den Objektivismus ${ }_{\mathrm{NEC}}$ vorgebrachten Einwände nicht auf den Primitivismus übertragen werden, da letzterer Farben nicht als physikalisch beschreibbare Eigenschaften auffassen wird und gerade diese sich einer möglichen Erklärung der Phänomenalität in den Weg stellen. Dagegen besitzen primitive Farben einfach eine objektive und phänomenale Natur. Aus demselben Grund ist auch die Annahme der Notwendigkeitsthese unproblematisch: selbst wenn Qualia-Inversionen oder ähnliche Fälle hinsichtlich der Reflektanzeigenschaften von Gegenständen möglich sind, bleiben Quale und primitive Farbe immer notwendigerweise korreliert: denn laut Primitivismus geben allein die objektiven, phänomenalen Farben die Phänomenalität der Farbqualia vor. Gerade die Möglichkeit, die Phänomenalität und die Repräsentationalität miteinander auf unkomplizierte Weise zu vereinen, macht die Attraktivität des Primitivismus aus. Er ist, auch wörtlich genommen, auf den ersten Blick die „einfachste“ Theorie der Farben; oder wie sie John Campbell (1993) im Ti- 
tel seines Aufsatzes benennt: A Simple View of Colour.

\title{
4.7.1. Der dualistische Primitivismus
}

Eine Möglichkeit, den Primitivismus für Farben einzuführen, besteht darin (ohne deren Objektivität dabei aufzugeben), jegliche Beziehung der Farben zu physikalisch (oder anderweitig naturwissenschaftlich) beschreibbaren Charakteristika zu leugnen und sie jenseits aller Physik-Immanenz - einschließlich der Supervenienz auf physik-immanenten Eigenschaften - anzusiedeln. Hardin grenzt diese Variante des Primitivismus gegen alle anderen Formen des Farbobjektivismus wie folgt ab: ${ }^{43}$

\begin{abstract}
„The objectivist can adopt two strategies. The first is to suppose that physical obejcts... are, at the level relevant to scientific theories of color, what the physicist says they are and that color must therefore be a physical property or combination of physical properties or else be supervenient on some set of physical properties. This is main-line objectivism... A minority opinion... has it that color is an objective property of physical objects over and above the properties with which the physicist endows them and that it is in no way reducible to or supervenient upon those properties.“ (Hardin (1988): 60)
\end{abstract}

Zuerst einmal stelt sich natürlich die Frage nach diesem „Jenseits“ der ontologischen Natur der „dort“ befindlichen Eigenschaften. Der Primitivist wird selbstverständlich hierauf keine Antwort geben können oder auch müssen, da er ja gerade die Analysierbarkeit der Farben ablehnt. Doch er muß sich, jedenfalls dieser Variante entsprechend, den Vorwurf gefallenlassen, einen Eigenschaftsdualismus einzuführen, da Farben keine physikalisch beschreibbare Natur aufweisen, aber trotzdem raum-zeitlich instantiiert sein sollen. Und dies wird ihn - abgesehen von der generellen Unplausibilität einer solchen Position - vor eine unüberwindliche, epistemische Schwierigkeit stellen (vgl. Hardin (1988): 60f.). Denn wenn keinerlei ontologische Abhängigkeit zwischen Farben einerseits und den physik-immanenten, für Farbwahrnehmungen kausal wirksamen Eigenschaften von Ge-

43 Hardin schreibt diese Position James Cornman zu, doch Byrne und Hilbert bezweifeln diese Zuordnung: für sie ist Cornman voraussichtlich eher ein physikalistischer Primitivist (Einleitung zu Byrne \& Hilbert (1997a): Fn. 32). 
genständen andererseits besteht, dann können beide auch vollkommen unabhängig voneinander instantiiert sein. Das heißt, ein Gegenstand kann sowohl eine Farbe besitzen, ohne daß er in uns irgendwelche Farbeindrücke hervorrufen kann, als auch Reflektanzeigenschaften aufweisen, die in uns Farbwahrnehmungen bewirken, ohne daß gleichzeitig eine Farbe von dem betreffenden Objekt instantiiert wird. Es macht also keinen Unterschied für unsere visuellen Wahrnehmungen - und somit auch für unsere daraus gewonnenen Überzeugungen über die präsentierten Eigenschaften -, ob die wahrgenommenen Gegenstände Farben haben oder nicht. Keith Campbell beschreibt die Position und ihre epistemische Schwierigkeit wie folgt: ${ }^{44}$

„The most straightforward view of colours is that they are what they seem, to the rather unreflective mind, on most normal occasions. On this view colours are properties in their own right, most often properties of surfaces of solid objects. Colours exist - alongside sizes, shapes and hardnesses - as independent, sui generis, physical, observer-independent characteristics.

Such a view suffers, inter alia, from terminal epistemic incredibility. Colours, so interpreted, play no role in our perception of colour, which is mediated exclusively by the frequency and intensity of light waves. So there is no way in which the perceiving mind could be given its grasp of these independent physical colours features.“ (K. Campbell (1993): 252)

Dadurch, daß Farben nicht mehr in unseren Farbwahrnehmungen präsentiert werden, wird aber selbst das Grundprinzip (IND) verletzt, und unsere Intuitive Farbkonzeption kann sich gar nicht auf die primitiven Farben beziehen. Die Hauptmotivation für die Akzeptanz eines Primitivismus geht somit verloren. Es ist uns gar nicht möglich, von den fraglichen Farbeigenschaften irgendwelche Kenntnis zu erlangen - vorausgesetzt, die Physik der Wechselwirkung von Licht und Materie ist, erstens, korrekt; und das Licht ist, zweitens, das einzige und auch hinreichende Medium, um die benötigten Informationen über die Farbigkeit von externen Gegenständen

44 Keith Campbell geht, wie das Zitat zeigt, davon aus, daß solche primitiven Eigenschaften physikalisch sein können, auch wenn sie keine ontologische Abhängigkeit zu anderen, physikalisch beschreibbaren Eigenschaften aufweisen. Doch ganz davon abgesehen, ob dies wirklich möglich ist, weist der Kontext eindeutig daraufhin, daß Campbell hier auf einen dualistischen Primitivismus Bezug nimmt. 
dem Auge zu vermitteln. Nur wenn eine der beiden letztgenannten Thesen gegen alle Einsicht geleugnet wird, ist der dualistische Primitivismus als eine Theorie der Farben haltbar. Sonst ergibt sich die Konsequenz, daß unsere Farbwahrnehmungen nicht wirklich Farbwahrnehmungen sind. Denn die absolut kontingente Verbindung von Farben und Farbwahrnehmungen kann dann keine Repräsentationsrelation etablieren, welche immer eine nomologische Korrelation voraussetzt. Doch es scheint, gerade vor dem Hintergrund eines ontologischen Realismus und eines Physikalismus, angebracht zu sein, sowohl den dualistischen Charakter abzulehnen als auch die physikalische Theorie über die Erzeugung von Sinneserfahrungen beizubehalten. Der dualistische Physikalismus bietet damit keine wirkliche Alternative zu den materialistischen Analysen der Natur von Farben.

\subsubsection{Der physikalistische Primitivismus}

So ist es auch nicht überraschend, daß die meisten Primitivisten eine gemäBigtere Variante ihrer Position annehmen, die grundsätzlich die Supervenienz von Farbtatsachen auf physikalisch beschreibbaren Tatsachen akzeptiert. ${ }^{45}$ In letzter Zeit ist diese Position vor allem von John Campbell vertreten worden, der insbesondere die darin enthaltene Objektivität und Primitivität betont und gleichzeitig den eben vorgebrachten Einwand gegen einen dualistischen Primitivismus berücksichtigt, indem er für Farben auch eine Erklärungskraft fordert:

„The view of colours as mind-independent must acknowledge some role for colours in colour-perception. [...] This is not a kind of physicalism about colours. To suppose that it must be is to assume an identification of the physical and the ob-

45 Neben John Campbell ist neuerdings Allen (2007) ein Vertreter dieser Position. Byrne und Hilbert rechnen Jonathan Westphal (1991) und, aller Wahrscheinlichkeit nach, James Cornman zu den physikalistischen Primitivisten (vgl. Einleitung zu Byrne \& Hilbert (1997a): Fn. 32, 33). Keith Campbell nennt zusätzlich noch James Ward (K. Campbell (1993): 252f.). Außerdem macht Broackes die Möglichkeit einer primitivistischen Theorie stark, auch wenn er sich die Option einer physikalistischen Analyse offenhält (vgl. Broackes (1992): Fn. 36; 447; 459). Er nennt zudem Hacker, Stroud und Putnam als Vertreter einer ähnlichen Position (vgl. Broackes (1992): Fn. 36). 
jective which the thesis may question. It may instead be that the characters of the colours are simply transparent to us. [...] Nevertheless, without there being a commitment to any thesis of property identity, someone who holds this simple view may acknowledge that colours are supervenient upon physical properties, if only in the minimal sense that two possible worlds which share all their physical characteristics cannot be differently coloured.“(J. Campbell (1993): 258)

Der hier beschriebene Primitivismus will möglichst nicht nur alle fünf Merkmale des repräsentationalen Aspektes und auch die Phänomenalität der Farben aufrechterhalten, sondern er möchte ebenso in jedem Fall die Analyse der Farben durch andere Eigenschaften vermeiden. Vor allem ist Objektivität nicht mit Physikalität gleichzusetzen: auch primitive Eigenschaften können objektiv instantiiert sein. Und trotzdem ist die Supervenienz auf physikalisch beschreibbare Eigenschaften erforderlich, da sonst die Repräsentiertheit und die damit verbundene Erklärungskraft der Farben nicht gewährleistet dies. Sobald aber eine - noch so beschaffene - ontologische Abhängigkeit zwischen Farben und den normalen Ursachen von Farbwahrnehmungen besteht, kann es auch eine nomologische Korrelation zwischen den Farben und entsprechenden Sinneserfahrungen geben. Damit entgeht der physikalistische Primitivismus dem gegen seine dualistische Variante vorgebrachten Einwand. Die Erklärungskraft kann zwar nicht kausaler Natur sein, dafür aber durch die Repräsentationsrelation gestützt werden. Der Epiphänomenalitätsvorwurf trifft den physikalistischen Primitivismus ebensowenig wie den Schwachen Physikalismus.

Aber es werden genug andere Einwände gegen den Primitivismus erhoben. Ein wesentlicher von diesen stützt sich auf der Überzeugung, daß die These (OS) auf Farben nicht zutreffen könnte, da sie unhaltbare Konsequenzen mit sich brächte. Michael Smith erläutert dies am Beispiel von Schmerzen, für die nach einer traditionellen Auffassung die Offensichtlichkeitsthese (welche er als transparency bezeichnet) gilt; dabei ist es für das Argument ganz unwichtig, ob dies tatsächlich auf Schmerzen zutrifft oder nicht.

„However, in the case of pain, such transparency comes as part of a package deal. Not only does a painful experience give us knowledge of which property pain is, we also know that something felt as pain is a pain and that something not felt as pain is not a pain. Transparency explains these consequences. For once we know 
which property pain is we know that there is nothing for a pain to do but to manifest itself in experience. There is nothing for an unexperienced pain to be. And nor is there any room for a state that masquerades as pain." (Smith (1993): 274)

Die Offensichtlichkeitsthese scheint also zu implizieren, daß weder Fehlrepräsentationen von Schmerzen, noch faktisch nicht-wahrgenommene Schmerzen möglich sind: ein Schmerz liegt immer nur genau dann vor, wenn ein Schmerz auch wirklich erlebt wird. Wenn dies so stimmt, dann läßt sich die These (OS) tatsächlich für objektiv verstandene Farben nicht aufrechterhalten.

„The folk allow that we can misperceive color, that color illusion is possible. But that is to draw a distinction between colors as they really are and colors as they appear to be, and that is to concede that the colors have a nature that outruns our experience of them." (Jackson (1996): 211)

Doch der Einwand von Smith und Jackson ist fehlerhaft, da sie zwei Ebenen von Eigenschaften miteinander verwechseln: zum einen sind uns in Farbwahrnehmungen Farbtatsachen, das heißt, Instantiierungen von Farben als Eigenschaften erster Ordnung gegeben; zum anderen jedoch auch - wenigstens laut der Offensichtlichkeitsthese - die Natur der Farben, welche sich aus Eigenschaften zweiter Ordnung zusammensetzt. Die Ambiguität kann wohl damit erklärt werden, daß die Rede von repräsentierten Eigenschaften oftmals mehr oder weniger auf zwei Weisen verstanden werden kann: entweder werden Gegenstände als Instanzen von bestimmten Eigenschaften repräsentiert, oder aber die Eigenschaften selbst werden als Instanzen von Merkmalen höherer Ordnung ,repräsentiert“. Es sollte klar sein, daß Sinneserfahrungen sich in erster Linie auf Gegenstände als Eigenschaftsträger beziehen: die möglichen Referenten unserer Farbwahrnehmungen sind Einzeldinge. Hinsichtlich deren Instantiierungen von Farbeigenschaften kann der Primitivist sowohl die Möglichkeit von Fehlrepräsentationen als auch die Möglichkeit des faktischen Nicht-Wahrgenommenwerdens behaupten, da einerseits primitive Farben immer objektiv instantiiert sind und andererseits die Einführung von Normalbedingungen, unter denen die primitiven Farben mit den entsprechenden Farbwahrnehmungen nomologisch korreliert sind, Abweichungen von veridischen Re- 
präsentationen erlauben.

Gerade in diesem Punkt zeigt sich, daß die Analogie mit den Schmerzen nicht zutrifft, da die erwähnte, traditionelle Auffassung eigentlich so zu verstehen ist, daß einen Schmerz zu instantiieren und einen Schmerz phänomenal zu erleben ein- und dasselbe sind: daß also der Träger des Schmerzes und die phänomenal bewußte Schmerzempfindung (wenn man so will) in Wirklichkeit identisch sind. Erst dann macht es Sinn, zu behaupten, daß Schmerztatsachen nur genau dann bestehen, wenn sie auch erlebt werden (will eine Sinnesdatentheorie nicht akzeptieren). Und dies ist bei objektiven Farben selbstverständlich nicht der Fall: eine Farbe zu instantiieren ist etwas ganz anderes, als eine Farbe sinnlich wahrzunehmen. ${ }^{46}$ Nun kann natürlich dem Primitivisten immer noch die Frage gestellt werden, ob er sich den wenigstens über die Natur der Farben, wie sie ihm introspektiv zugänglich ist, irren kann. Doch auch dies ist ihm möglich - ja muß ihm sogar möglich sein -, da Introspektion nicht infallibel sein kann (vgl. Shoemaker (1994a); (1994c)).

Die Offensichtlichkeitsthese fordert dementsprechend nicht die repräsentationale Veridizität unserer Farbwahrnehmungen, und auch nicht die Veridizität unserer Introspektionen auf Farbwahrnehmungen, sondern nur über die Gültigkeit unserer introspektiv gewonnenen Intuitiven Farbkonzeption. Sie ist insbesondere keine epistemologische, sondern nur eine on-

46 Auch Jackson erkennt dies an, ohne interessanterweise daraus die Konsequenz zu ziehen, daß Farben laut einem Vertreter der Offensichtlichkeitsthese fehlrepräsentiert werden können (dieser Umstand läßt sich vielleicht dadurch erklären, daß er gegen einen subjektivistischen Befürworter dieser These argumentiert):

„Intuition requires treating our experience of color as typically caused by color, and it is part of the... notion of causation that causes and effects are distinct. But if our experience of color is distinct from what it is an experience of, how could it be transparently reveal the nature of color?" (Jackson (1996): 211)

Jackson stellt hier zwar die Transparenzintuition in Frage, ohne jedoch ein Argument zu liefern. Epistemisch betrachet ist die Transparenz oder Offensichtlichkeit von Eigenschaften vor dem Hintergrund einer direkt-realistischen und externalistischen Wahrnehmungstheorie gar nicht so sehr das Problem. Vielmehr scheint hier implizit auf das allgemeinere Problem verwiesen zu werden, wie überhaupt Wissen von geistunabhängigen Tatsachen in der Außenwelt erlangt werden kann. 
tologische These. Man kann ohne weiteres ein rotes Objekt fälschlicherweise als grün wahrnehmen, und dabei trotzdem die Natur der Farbe Grün aufgrund von Introspektion korrekt erkennen; und es ist ebenso möglich (wenn auch seltener), eine Grünwahrnehmung fehlerhaft zu introspektieren und aufgrunddessen der Farbe Grün Merkmale zuzuschreiben, die sie gar nicht hat. Aber im Normalfall sind sowohl unsere Wahrnehmungen von Farbtatsachen als auch unsere Introspektion auf die Wahrnehmungen zutreffend, so daß wir verläßliches Wissen darüber erhalten können, welche Gegenstände grün sind und welche Wesensmerkmale der Farbe Grün eigen sind. Die vorgebrachten Einwände von Smith und Jackson treffen den Primitivismus nicht, da dieser weder behauptet, noch behaupten muß, daß Farben die Referenten unserer Farbwahrnehmungen sind: sie werden vielmehr repräsentiert und können von den Referenten instantiiert sein.

Nun bringt Smith noch einen weiteren Kritikpunkt vor. Campbell sieht primitive Farben als Basiseigenschaften der über Farbwahrnehmungen individuierten Farbdispositionen $\left(\mathrm{D}_{\mathrm{M}}\right)$ an. Die Motivation hierfür liegt in dem Umstand begründet, daß Campbell Farben als objektive Eigenschaften ansehen möchte, die zudem eine Erklärungsfunktion hinsichtlich von Farb wahrnehmungen besitzen. Da die betreffenden Farbdispositionen geistabhängig sind, und da neben den Dispositionen selbst vermutlich nur noch die Basiseigenschaften eine erklärende Rolle für unsere Sinneserfahrungen spielen können, kommt Campbell dazu, Farben mit letzteren zu identifizieren (vgl. J. Campbell (1993): 258). Smith wirft Campbell nun vor, daß er kein Argument dafür anbieten kann - außer dem Wunsch, die Objektivität erhalten zu wollen -, nicht doch die subjektivistischen Dispositionen als Farben anzunehmen. Zudem führt er sogar ein Argument gegen die Kategorialisierung von primitiven Farben an: denn während Formen objektiv und unabhängig von unseren Wahrnehmungen gemessen werden können, trifft dies auf primitive Farben anscheinend nicht zu, weil sie nicht durch andere Eigenschaften erfaßt oder analysiert werden können (vgl. Smith (1993): 217f.). Damit kann vermutlich die aufgrund phänomenologischer Betrachtungen behauptete Ähnlichkeit von Formen und Farben, die als ein Motivationsgrund für die Annahme des Primitivismus als objektivistische Theorie dient, wohl durchbrochen werden. 
"The objection is that we have no understanding of what it would be for colour to be a categorical property of objects in the spirit of the simple view. For that would require that we have some independent grip on what it is for an object to be coloured, an independent grip that simply isn't to be had if we are also to imagine, as the simple view demands, that the 'real nature' of colour is 'transparent' to us in colour experiences. [...] In the end, it seems to me that the simple view of colour is dubiously coherent." (273)

Die Kategorialität von primitiven Farben wird vor allem deshalb fraglich, weil sie auf physikalisch beschreibbaren Eigenschaften supervenieren müssen, ohne jedoch auf diese reduziert oder durch diese analysiert werden zu dürfen. Eine Möglichkeit, dieser Forderung gerecht zu werden, ist eben die Identifikation mit Dispositionen. Campbell hat diese nun vermieden, um nicht einen Subjektivismus akzeptieren zu müssen. Aber auch wenn er es selbst nicht in Betracht zieht, ist es für ihn theoretisch möglich, wie Byrne und Hilbert Farben als objektivistische Dispositionen im Sinne von $\left(\mathrm{D}_{\mathrm{vs}}\right)$ anzusehen und zusätzlich die Notwendigkeitsthese anzunehmen, um die Aufrechterhaltung der Offensichtlichkeitsthese gewährleisten zu können. Der Primitivismus hat dann gegenüber dem Schwachen Physikalismus sogar den Vorteil, daß er keine Schwierigkeiten bei der Erklärung der Farbphänomenalität bekommt. Denn während physikalisch beschreibbare Eigenschaften nicht dieselbe phänomenal Struktur aufweisen können wie die Farbqualia, ist dies für primitive Eigenschaften per definitionem kein Problem: primitive Farben sind phänomenal und bestimmen die Phänomenalität der Farbqualia. Zudem wird der Primitivismus auch dann noch dem (wenn auch nicht so wichtigen) Einfachheitsmerkmal gerecht. Demnach ist es angebracht, primitive Eigenschaften als Dispositionen anzusehen.

Es gibt übrigens auch keine zufriedenstellende Alternative zu einem objektivistisch-dispositionalistischem Primitivismus. Die einzige Möglichkeit, irreduzibel supervenierende Eigenschaften als kategoriale Bestandteile der Welt zu erhalten, wäre, ihnen einen vergleichbaren Status zuzuschreiben, wie ihn der anomale Monismus den mentalen Eigenschaften zukommen läßt. Doch dies bringt nicht nur einen kategorialen Unterschied in den Naturen von Farben und Formen mit sich (womit wiederum eine der Hauptmotivationen für den Primitivismus weggefällt), sondern es führt auch erneut zu einem Eigenschaftsdualismus mit den oben genannten, epi- 
stemischen Unzulänglichkeiten. Nun ist der dispositionalistische Primitivismus aber selbst problematisch. Man könnte meinen, daß er gegen die Nicht-Analysierbarkeitsforderung verstößt; doch dies ist nicht der Fall, da die Aussage, daß primitive Farben dispositionale (oder auch: kategoriale) Eigenschaften sind, einfach eine Konzeption von Eigenschaften der anderen vorzieht. Doch hierin mag gerade ein Problem liegen. Da der Primitivismus Farben als irreduzible Eigenschaften sui generis ansieht, ist es wohl kaum zu vermeiden, daß die Farbdispositionen als genuine Eigenschaften anzusehen sind, für die der ontological free lunch nicht gilt. Damit würden Farbtatsachen, zusätzlich zu ihren Realisatoren, etwas zur Welt hinzuaddieren; und der Primitivismus bekäme das bereits bekannte Problem, sowohl von kontingenten Kausalgesetzmäßigkeiten als auch von notwendigen Farbgesetzmäßigkeiten in der Natur ausgehen zu müssen (vgl. Armstrong (1997): 83f.; 250ff.). Zudem wäre es dann fragwürdig, warum gerade Farben diese herausragende Stellung innerhalb der Realität einnehmen; und ob es sich nicht doch wieder um einen Eigenschaftsdualismus handeln würde.

Aber auch wenn primitive Dispositionen aus ontologischer Sicht doch kein Problem darstellen sollten, gibt es noch ein wichtigeres Argument gegen den Primitivismus, welches tatsächlich ganz unabhängig davon formuliert werden kann, ob primitive Farben als dispositionale oder als kategoriale Eigenschaften angesehen werden. Es handelt sich dabei um ein Problem, daß dem der kausalen Überdetermination sehr ähnlich ist. Die Wissenschaft der Farben hat gezeigt, daß die Reflektanzprofile SSR die einzig kausal wirksamen Eigenschaften der Gegenstände hinsichtlich des Auftretens von Farbwahrnehmungen sind. Zudem läßt sich zwischen den phänomenalen Typen der Sinneserfahrungen und den Reflektanztypen $S S R_{\mathrm{T}}$ eine nomologische Korrelation unter Normalbedingungen nachweisen. Während die Reflektanzprofile unsere Farbwahrnehmungen kausal erklären, tun dies die Reflektanztypen aufgrund der Repräsentationsrelation. Was können aber darüberhinaus noch primitive Eigenschaften erklären? Man könnte vielleicht annehmen, daß diese eins-zu-eins auf den Reflektanztypen supervenieren würden, so daß sie dadurch ebenfalls mit den Farbwahrnehmungstypen gesetzmäßig verbunden wären. Das hieße jedoch, daß Farb wahrnehmungen faktisch sowohl Reflektanztypen als auch primitive Farben repräsentierten und von beiden erklärt werden könnten. Aber dann 
würden die Farben vollkommen überflüssig sein, weil es ohne weiteres passieren könnte, daß eine Farbwahrnehmung vorläge, obwohl der wahrgenommene Gegenstand nur den entsprechenden Reflektanztyp, nicht aber auch die dazugehörige Farbe, instantiieren würde. Es läge dann eine Repräsentation vor, die zudem vollständig erklärt werden könnte. Für noch hinzukommende, primitive Farben gäbe es nichts zu tun. Zudem ergäbe sich ein neuerlicher Überflüssigkeitseinwand:

„The direct realist who rejects this identity view [that colours are (disjunctions of) physical properties - der Autor] will have to argue that the colours are distinct from, though correlated with, the physical processes. But now let such such a non-reductive direct realist consider the hypothesis that the colours as he or she conceives them (out in the world, unreduced) do not exist, but that the physical processes, laws, etc. are unchanged. Why would the experience of colour be any different from the experience actually had? Same causes and laws, same effects. This goes some way, not the whole way obviously, but some way, to suggest that a reductive account of colour ought to be correct.“ (Armstrong (1997): 59)

Der Primitivismus sollte diese beiden hypothetischen Möglichkeiten also leugnen. Insbesondere darf keine eins-zu-eins-Supervenienz zwischen Farben und Reflektanzeigenschaften bestehen. Vielmehr wird er plausibel zu machen versuchen, daß in bestimmten möglichen Welten Farben durch physikalische Eigenschaften realisiert werden, die von Reflektanzprofilen und -typen ganz verschieden sind und trotzdem Farbwahrnehmungen kausal hervorrufen können, so daß immer noch eine nomologische Korrelation zwischen Farben und Farbwahrnehmungstypen besteht. So ist es doch denkbar, daß in einer von der unseren recht weit entfernten Welt ganz andere, physikalische Gesetzmäßigkeiten gelten und die farbwahrnehmenden Subjekte über ganz andere, visuelle Sinnesorgane verfügen. In dieser Welt werden Farbwahrnehmungen demgemäß nicht mehr über Lichtreize hervorgerufen; und Farbtatsachen supervenieren dort nicht mehr auf Instantiierungen von Reflektanzen, sondern auf Instantiierungen anderer, physikalisch beschreibbarer Eigenschaften. Dementsprechend können also nur die Farben das Auftreten der entsprechenden Sinneserfahrungen erklären, nicht jedoch irgendwelche physikalischen Eigenschaften.

Doch in dieser Erwiderung stecken zwei Mängel. Erstens ist es sehr umstritten - insbesondere vor einer materialistischen Ansicht über die Rea- 
lität -, ob in solchen Fällen noch von Farbwahrnehmungen gesprochen werden sollte, weil Farbphänomene auf einmal vollkommen von Lichtphänomenen abgetrennt werden. Denn es ist fraglich, ob auch dann noch Farbtatsachen vorliegen können, wenn ein Farbigkeitsunterschied keinen Einfluß mehr auf das reflektierte Licht hat; ob Farbwahrnehmungen möglich sind, wenn sie die erforderliche Information nicht über Lichtwellen erhalten; und ob die betreffenden Subjekte deshalb noch als farbwahrnehmende gelten sollten. Hierauf mag der primitivistische Objektivist eventuell noch antworten, daß Licht aus ontologischer Sicht tatsächlich nichts mit Farben zu tun hat und daß unsere entsprechende, intuitive Meinung nicht zutrifft.

Aber selbst wenn diese Konzeption von Farben aufrechterhalten werden kann, bleibt immer noch, zweitens, der Umstand bestehen, daß Farbwahrnehmungen immer sowohl die primitiven Farben als auch die diese realisierenden, physikalisch beschreibbaren Eigenschaften repräsentieren: wie in der aktualen Welt, so auch in allen anderen Welten, in denen es Farbtatsachen und -wahrnehmungen gibt. Der Schritt von der aktualen Welt weg nützt also nichts: immer wird sich zusätzlich zu der Farbeigenschaft eine Klasse oder Typus von physik-immanenten Eigenschaften finden lassen, die unter den entsprechenden Normalbedingungen mit Farbwahrnehmungen nomologisch korreliert sind. In der aktualen Welt sind dies die Reflektanztypen; in anderen können es andere Eigenschaftsarten sein. Warum sollten also die entprechenden, physikalischen Eigenschaften jeweils wirklich von den Farben verschieden sein? Wie kann die Einführung von primitiven Eigenschaften vor dem Hintergrund einer drohenden Erklärungsdetermination noch sinnvoll gedeutet werden, vor allem, wenn die aktuale Welt als Maßstab genommen wird? Broackes stellt sich dieselbe Frage und weist zumindest auf die Plausibilität der Identifizierung von Farben mit physikalisch beschreibbaren Reflektanztypen oder „Weisen der Lichtbeeinflussung“ (ways of changing light) hin:

„Psychophysics and colorimetry have, I think, put us in a position where we can tell of any newly presented object what colour it is, simply from its spectral reflectance curve (together with standard data culled from human subjects). It should therefore be possible to find a 'physical' property coextensive with 'red'. The questions remain whether this physical property will be necessarily coextensive with the colour, and whether it will be identical with it - and I cannot pre- 
tend to answer these here. But it must be obvious that the view of colours as introduced by a distinctively subjective scheme of explanation, and as phenomenally characterized ways of changing light..., does nothing to rule out the idea that they might be characterizable a posteriori in other ways as well." (Broackes (1992): 459)

Es gibt aber noch eine letzte, entscheidende Schwierigkeit für den Primitivismus. Es sollte mittlerweile klar geworden sein, daß er, damit er überhaupt sinnvollerweise als Theorie der Farben in Betracht gezogen werden kann, die primitiven Farben als von den Farbwahrnehmungen repräsentierte Eigenschaften ansehen muß; andernfalls kann nicht einmal der Grundthese (IND) gerecht werden. Doch nun ist Repräsentation nichts anderes als nomologische Korrelation unter Normalbedingungen. Da eine eins-zu-einsSupervenienz zwischen Reflektanztypen und Farben unbedingt vermieden werden sollte, um nicht gleich der repräsentationalen Erklärungsüberdetermination zum Opfer zu fallen; und da deshalb Farbwahrnehmungen auch dann veridisch sein können, wenn überhaupt gar keine Reflektanztypen oder -profile involviert sind (sondern ganz andere, physikalische Eigenschaften), ist es dem Primitivismus nicht möglich, die Normalbedingungen vom Schwachen Physikalismus (wie sie auch in dem eigenen Abschnitt über Normalbedingungen beschrieben werden) zu übernehmen. Und es ist nicht ersichtlich, wodurch diese sinnvollerweise ersetzt werden könnten. In jedem Fall können die Normalbedingungen nicht über physikalisch beschreibbare Eigenschaften bestimmt werden. Und mithilfe der primitiven Farben ist eine substantielle Charakterisierung nicht möglich, gerade da primitive Eigenschaften nicht substantiell analysiert werden können. Der Primitivismus kann Normalbedingungen nur trivial bestimmen: es sind die Bedingungen, unter denen primitive Farben veridisch wahrgenommen werden. Wie zu der primitiven Natur der Farben kann hierzu darüberhinaus nichts mehr gesagt werden.

Dem Primitivismus ist es dementsprechend unmöglich, Normalbedingungen für die Repräsentation von primitiven Farben substantiell zu formulieren. Damit kann aber auch nicht mehr davon ausgegangen werden, daß unsere Farbwahrnehmungen diese Eigenschaften repräsentieren: denn die benötigte, nomologische Korrelation kann nicht nachgewiesen oder begründet werden, da die erforderlichen Normalbedingungen höchstens tri- 
vialerweise stipuliert werden können. Der Verdoppelung der Repräsentation und der damit verbundenen Überdetermination der repräsentationalen Erklärung kann der Primitivist wahrscheinlich ebenso nicht entgehen, ohne eine dualistische Position anzunehmen. Und letztere zeigt elementare Probleme mit der Erklärung der Beziehung zwischen Farbtatsachen und den auf diese Tatsachen gerichteten, mentalen Zuständen. Zudem muß sich der physikalistische Primitivismus mit den generellen, ontologischen Problemen irreduzibel supervenierender Eigenschaften auseinandersetzen, ob sie nun dispositional oder kategorial verstanden werden.

Damit stellt der Primitivismus - wenn er auch nicht unbedingt inkosistent ist - eine wenig plausible Theorie der Farben dar und sollte möglichst vermieden werden. Eine Alternative ist der subjektivistische Dispositionalismus, der Farben als Dispositionen zu farb-phänomenalen, mentalen $\mathrm{Zu}$ ständen im Sinne von $\left(\mathrm{D}_{\mathrm{M}}\right)$ ansieht. Und die objektivistische Position, die dem Vorschlag von Campbell am nächsten kommt, ist ganz klar der Notwendigkeitsobjektivismus, welcher auf dem Schwachen Physikalismus basiert. Bei der Wahl der zweiten Option wird dann natürlich die Offensichtlichkeitsthese durch die Transparenzthese ersetzt werden, da die Analysierbarkeit der Farben zugegeben wird. ${ }^{47}$ Der Primitivismus bezieht seine Attraktivität gerade aus dem Umstand, daß er sowohl den objektivistischen als auch den subjektivistischen Intuitionen und Ansichten über Farben gerecht werden möchte; aber er wird wohl aus genau demselben Grund keine zufriedenstellende Ontologie der Farben bereitstellen können.

47 Laut Byrne und Hilbert ist dies der Weg, den Jonathan Westphal beschreitet, da er anscheinend einen Primitivismus mit einer auf der Colorimetrie basierenden Analyse der Natur von Farben verbinden möchte (vgl. Einleitung zu Byrne \& Hilbert (1997a): xxvii). Wenn dies für Westphal zutreffen sollte, dann ist er nach meiner Klassifikation kein Primitivist mehr, sondern bereits ein Schwacher Physikalist, der wahrscheinlich - wie Byrne und Hilbert selbst - die Notwendigkeitsthese akzeptieren wird. Broackes Position stellt genau eine solche Modifikation der Ansicht von Westphal dar (vgl. Broackes (1992): 454ff.). 